\title{
Normal form of equivariant maps in infinite dimensions
}

\author{
Tobias Diez ${ }^{1}$ (D) $\cdot$ Gerd Rudolph ${ }^{2}$
}

Received: 12 November 2020 / Accepted: 6 May 2021 / Published online: 14 October 2021

(c) The Author(s) 2021

\begin{abstract}
Local normal form theorems for smooth equivariant maps between infinite-dimensional manifolds are established. These normal form results are new even in finite dimensions. The proof is inspired by the Lyapunov-Schmidt reduction for dynamical systems and by the Kuranishi method for moduli spaces. It uses a slice theorem for Fréchet manifolds as the main technical tool. As a consequence, the abstract moduli space obtained by factorizing a level set of the equivariant map with respect to the group action carries the structure of a Kuranishi space, i.e., such moduli spaces are locally modeled on the quotient by a compact group of the zero set of a smooth map. The general results are applied to the moduli space of anti-self-dual instantons, the Seiberg-Witten moduli space and the moduli space of pseudoholomorphic curves.
\end{abstract}

Keywords Submersion · Immersion · Group action · Equivariant map · Kuranishi structure $\cdot$ Moduli space $\cdot$ Anti-self-dual Yang-Mills · Seiberg-Witten ·

Pseudoholomorphic curves

Mathematics Subject Classification 58K70 -58D27 · 58D19 · 58G05 $\cdot$ 22E65 · 70S15

\section{Introduction}

Moduli spaces parametrize solutions of partial differential equations up to some natural notion of equivalence. They play an essential role in theoretical physics as well as in pure mathematics. In (gauge) field theory, they represent the reduced phase spaces after the (gauge) symmetry has been divided out, see [68] and references therein. The topological and inherently non-perturbative aspects of the physical system are often manifested in the geometry of the moduli space. On the other hand, the structure of the moduli space encodes some astounding topological and geometrical properties. The topological data extracted from this auxiliary moduli space serve as a powerful nonlinear invariant of the

Tobias Diez

T.Diez@tudelft.nl

Gerd Rudolph

rudolph@rz.uni-leipzig.de

1 Institute of Applied Mathematics, Delft University of Technology, 2628 XE Delft, Netherlands

2 Institut für Theoretische Physik, Universität Leipzig, 04009 Leipzig, Germany 
original manifold. Important examples can be found in many areas: gauge theory (flat connections [4], anti-self-dual instantons [18], Seiberg-Witten monopoles [73]), symplectic geometry (pseudoholomorphic curves [32], symplectic field theory [23]), complex geometry [46] and string theory.

For applications in both geometry and physics, a deep understanding of the local structure of the moduli space is essential. Of particular importance is the formation of singularities due to points having a non-trivial stabilizer under the symmetry group action. Although relying on similar techniques, so far these fundamental features are analyzed only on a case by case basis for each moduli space separately.

In this paper, we provide a general framework that gives a unified approach to these differential-geometric moduli spaces. Specifically, we establish a convenient normal form for a large class of nonlinear differential equations with symmetries. Furthermore, we show that the corresponding moduli space of solutions can be endowed with the structure of a Kuranishi space, which roughly speaking means that it can be locally identified with the quotient of the zero set of a smooth map by the linear action of a compact group. Our approach is inspired by the ideas that underlie the Lyapunov-Schmidt reduction for dynamical systems and the Kuranishi method for moduli spaces in differential geometry. As we discuss in the last section, our general framework applies to the fundamental moduli spaces of anti-self-dual instantons, Seiberg-Witten monopoles and pseudoholomorphic curves, leading to simplified and unified proofs that these moduli spaces have natural Kuranishi charts.

Our results are phrased in terms of smooth equivariant maps between infinite-dimensional manifolds modeled on locally convex spaces. In field theory and global analysis, the maps under consideration are usually given by partial differential operators between spaces of sections. As such, they give rise to smooth maps between appropriate Sobolev completions. On the other hand, the symmetry action often involves compositions of maps and thus fails to be differentiable as a map between spaces of sections of a given Sobolev class. For example, the group of diffeomorphisms of a fixed Sobolev regularity is a Banach manifold as well as a topological group but not a Lie group, because the group operation is not differentiable. When working with smooth objects these problems disappear, and the group of smooth diffeomorphisms is a bona fide Lie group modeled on a Fréchet space. In order to include these important cases, we consider throughout the paper infinite-dimensional manifolds modeled on Fréchet or even more general locally convex spaces. The approach via Fréchet spaces has also the advantage that the geometric arguments in the applications are simpler, because one does not have to deal with issues originating in the low regularity of the sections or in the loss of differentiability.

Beyond the Banach context, the classical Banach inverse function theorem used in the proof of Theorem 3.5 has to be replaced by a different version. We will use Glöckner's inverse function theorem for maps between Banach spaces with parameters in a locally convex space and the Nash-Moser theorem in the tame Fréchet setting. Moreover, the normal form theorems are phrased and proved in a modularized way, leading to a flexible general framework in which other analytical setups can be included in a "plug and play" fashion based on other inverse function theorems.

As another approach to the issues that composition is not smooth relative to a fixed Sobolev regularity, Hofer, Wysocki and Zehnder have introduced the scale calculus, see, e.g., [40, 41] and references therein. In this approach, one works with a sequence of Banach spaces, while the Nash-Moser approach focuses on the limit space, see [28] for a detailed comparison. The scale calculus is tailored to the elliptic setting one encounters in symplectic field theory. We emphasize that the Nash-Moser theorem covers these cases as 
well, see Sects. 3.4 and 5.5, but additionally allows for applications that go well beyond the elliptic setting. This will be crucial for problems where classical Banach space methods do not apply and more sophisticated Nash-Moser arguments are needed, such as deformation and normal form problems in the theory of fibrations [34] and Poisson manifolds [11, 48]. Finally, the general framework developed in this paper lays the foundation for the singular symplectic reduction in infinite dimensions, which will be presented elsewhere $[13,14]$.

The paper is structured as follows.

Section 2 We begin by considering the linear setting and determine under which conditions a continuous linear map between locally convex spaces can be brought into a normal form; that is, it factorizes through a topological isomorphism. An operator admitting such a factorization is called regular. Fredholm operators, and in particular elliptic operators, are important examples of regular operators. As a preparation for the nonlinear case, we also discuss regularity of families of linear maps depending continuously on a parameter and of chain complexes.

Section 3 Next, we discuss the local behavior of a smooth map between locally convex manifolds. Unifying the concepts of immersion, submersion and subimmersion in one framework, the notion of a normal form of a nonlinear map is introduced. Using versions of the inverse function theorem, we establish Theorems 3.5, 3.7, 3.8, 3.11 and 3.12 which show that a given map can be brought into such a normal form in various functional-analytic settings under suitable conditions. These normal form theorems provide a unified approach to the immersion theorem, the level set theorem and the constant rank theorem in the setting of locally convex manifolds and tame Fréchet manifolds.

Section 4 We introduce the concept of an equivariant normal form and provide suitable conditions which ensure that an equivariant map can be brought into such a normal form, resulting in Theorem 4.6 and its variants. Besides the normal form results in Sect. 3, the main technical tool is the existence of slices for actions on Fréchet manifolds as established in [16]. We investigate the local structure of the moduli space obtained by taking the quotient of a level set of the equivariant map by the group action. Under the assumption that the map can be brought into an equivariant normal form, the corresponding moduli space has the structure of a Kuranishi space, which roughly speaking means that it can be locally identified with the quotient of the zero set of a smooth map with respect to the linear action of a compact group. Moreover, we find additional conditions on the normal form which ensure that the moduli space is stratified by orbit types. Finally, to show the utility of this novel framework, we apply the general theory to the example of the moduli space of antiself-dual Yang-Mills connections, to the Seiberg-Witten moduli space and to the moduli space of pseudoholomorphic curves. The upshot is that these moduli spaces have natural Kuranishi charts. Since no Sobolev techniques are used, the arguments are streamlined and simplified. For examples, we do not face any issues coming from functions of low regularity that complicate the analysis in the usual Banach approach.

In Appendix, we summarize without proofs the relevant background material concerning the calculus of infinite-dimensional manifolds with a primary focus on the inverse function theorem as well as on Lie group actions.

Most of the material first appeared in the first author's thesis [13].

\subsection{Conventions}

Our main references for terminology and notation in the framework of infinite-dimensional differential geometry are [35] for the tame Fréchet category and [57] for the general 
locally convex case. As the latter is our standard setting, we use the word "manifold" to refer to an infinite-dimensional locally convex manifold; further assumptions on the model space are designated by additional qualifier such as "Fréchet or finite-dimensional manifold." For a Lie group action $\Upsilon: G \times M \rightarrow M$, we also use the "dot notation" and abbreviate $\Upsilon(g, m) \equiv g \cdot m$ for $g \in G$ and $m \in M$. On the infinitesimal level, for $A \in \mathfrak{g}$, we write the fundamental vector field $A^{*}$ as $A_{m}^{*}=\mathrm{T}_{e} \Upsilon_{m}(A) \equiv A . m$. Similarly, $\mathrm{T}_{m} \Upsilon_{g}(X) \equiv g . X$ for $X \in \mathrm{T}_{m} M$.

\section{Normal form of a linear map}

In this section, we discuss the normal form of continuous linear maps between locally convex spaces. Recall that every $(m \times n)$-matrix $T$ with rank $r$ can be written in the following normal form

$$
T=P\left(\begin{array}{cc}
0 & 0 \\
0 & 1_{r \times r}
\end{array}\right) Q,
$$

where $P$ and $Q$ are invertible matrices of type $(m \times m)$ and $(n \times n)$, respectively. As we will see, a similar factorization is possible for continuous linear maps between locally convex spaces, which are relatively open and whose kernel and image are closed complemented subspaces. We call such operators regular and their associated representation (2.1) a normal form. As a preparation for the nonlinear case, we define and study regularity of families of linear maps depending continuously on a parameter. With a view toward applications, we give a brief overview of the theory of Fredholm operators and of elliptic operators in the locally convex framework and, in particular, show that these operators are regular.

\subsection{Uniform regularity}

Let $X$ and $Y$ be locally convex spaces, and let $P$ be a neighborhood of 0 in some locally convex space. A continuous map $T: P \times X \rightarrow Y$ is called a continuous family of linear maps if, for all $p \in P$, the induced map $T_{p} \equiv T(p, \cdot): X \rightarrow Y$ is linear.

Definition 2.1 A continuous family $T: P \times X \rightarrow Y$ of linear maps between locally convex spaces $X$ and $Y$ is called uniformly regular (at 0 ) if there exist topological decompositions

$$
X=\operatorname{Ker} T_{0} \oplus \operatorname{Coim} T_{0}, \quad Y=\operatorname{Coker} T_{0} \oplus \operatorname{Im} T_{0},
$$

where Coim $T_{0}$ and Coker $T_{0}$ are closed subspaces ${ }^{1}$ of $X$ and $Y$, and, for every $p \in P$, the map $\tilde{T}_{p}=\operatorname{pr}_{\operatorname{Im} T_{0}} \circ\left(T_{p}\right)_{\uparrow \operatorname{Coim} T_{0}}: \operatorname{Coim} T_{0} \rightarrow \operatorname{Im} T_{0}$ is a topological isomorphism and the inverses $\tilde{T}_{p}^{-1}$ form a continuous family $P \times \operatorname{Im} T_{0} \rightarrow$ Coim $T_{0}$ of isomorphisms.

\footnotetext{
${ }^{1}$ The coimage and cokernel of an continuous linear map $T: X \rightarrow Y$ are defined as $\operatorname{Coim} T=X / \operatorname{Ker} T$ and Coker $T=Y / \operatorname{Im} T$, respectively. There exists, of course, no canonical realization of these quotient spaces as subspaces of $X$ and $Y$. Nonetheless, the choice of topological complements $A$ and $B$ of $\operatorname{Ker} T$ and $\operatorname{Im} T$, respectively, leads to the identifications $\operatorname{Coim} T \simeq A$ and Coker $T \simeq B$. It is in this sense and with a slight abuse of notation that we view Coim $T$ and Coker $T$ as subspaces of $X$ and $Y$, respectively.
} 
In the case $P=\{0\}$, the notion of uniform regularity reduces to the notion of a relatively open operator $T: X \rightarrow Y$ with closed complemented kernel and image. We refer to this situation by saying that $T$ is a regular operator. ${ }^{2}$ Every regular operator $T$ can be written in a normal form similar to (2.1):

$$
T=P\left(\begin{array}{ll}
0 & 0 \\
0 & \tilde{T}
\end{array}\right) Q
$$

where $Q: X \rightarrow \operatorname{Ker} T \oplus \operatorname{Coim} T$ and $P: \operatorname{Coker} T \oplus \operatorname{Im} T \rightarrow Y$ are the natural isomorphisms determined by the decompositions (2.2), and $\tilde{T}: \operatorname{Coim} T \rightarrow \operatorname{Im} T$ is a topological isomorphism. We call $\tilde{T}$ (together with the isomorphisms $Q$ and $P$ ) a normal form of $T$.

If the space of invertible maps is open in the space of all continuous linear maps, then, for every continuous family $T: P \times X \rightarrow Y$ with $T_{0}$ being regular, one can shrink $P$ to pass to a uniformly regular family. This openness property fails when one leaves the Banach realm. However, when it does hold, uniform regularity reduces to a condition at one point.

Lemma 2.2 Let $T: P \times X \rightarrow Y$ be a continuous family of linear maps between locally convex spaces $X$ and $Y$. If $\operatorname{Im} T_{0}$ is finite-dimensional, then $T$ is uniformly regular after possibly shrinking $P$.

Proof Since $T_{0}$ has a finite-dimensional range, by [45, Proposition 20.5.5], $\operatorname{Im} T_{0}$ is closed and has a topological complement. Moreover, $\operatorname{Ker} T_{0}$ has finite codimension in $X$ and hence is topologically complemented according to [45, Proposition 15.8.2]. The maps $\tilde{T}_{p}:$ Coim $T_{0} \rightarrow \operatorname{Im} T_{0}$ are continuous linear maps between finite-dimensional spaces. Since $\tilde{T}_{0}$ is a bijection, the openness of the set of invertible operators implies that $\tilde{T}_{p}$ is a topological isomorphism for $p \in P$ close enough to 0 .

Uniform regularity implies a semi-continuity property of the kernel and the image. Similar semi-continuity properties are well known for families of Fredholm operators between Banach spaces [42, Corollary 19.1.6].

Lemma 2.3 Let $T: P \times X \rightarrow Y$ be a continuous family of linear maps between locally convex spaces $X$ and $Y$. If $T$ is uniformly regular, then the following holds:

1. The kernel of $T$ is upper semi-continuous at 0 in the sense that $\operatorname{Ker} T_{p} \subseteq \operatorname{Ker} T_{0}$ for all $p \in P$.

2. The image of $T$ is lower semi-continuous at 0 in the sense that $\operatorname{Im} T_{p} \supseteq \operatorname{Im} T_{0}$ for all $p \in P$.

Proof The inclusions $\operatorname{Ker} T_{p} \subseteq \operatorname{Ker} T_{0}$ and $\operatorname{Im} T_{p} \supseteq \operatorname{Im} T_{0}$ need to be valid, because otherwise $\tilde{T}_{p}=\operatorname{pr}_{\operatorname{Im} T_{0}} \circ\left(T_{p}\right)_{\left\lceil\operatorname{Coim} T_{0}\right.}$ cannot be an isomorphism from Coim $T_{0}$ to $\operatorname{Im} T_{0}$.

Uniform regularity is tightly connected to the invertibility of an extended operator.

${ }^{2}$ We use the word "operator" interchangeably with "continuous linear map." 
Theorem 2.4 Let $T: P \times X \rightarrow Y$ be a continuous family of linear maps between locally convex spaces $X$ and $Y$. Then, the following are equivalent:

1. T is uniformly regular.

2. There exist locally convex spaces $Z^{ \pm}$, continuous linear maps $T^{+}: Z^{+} \rightarrow Y$ and $T^{-}: X \rightarrow Z^{-}$, and continuous families of linear maps $S: P \times Y \rightarrow X, S^{-}: P \times Z^{-} \rightarrow X$, $S^{+}: P \times Y \rightarrow Z^{+}$and $S^{-+}: P \times Z^{-} \rightarrow Z^{+}$with $S_{0}^{-+}=0$ such that

$$
\left(\begin{array}{cc}
T_{p} & T^{+} \\
T^{-} & 0
\end{array}\right)^{-1}=\left(\begin{array}{cc}
S_{p} & S_{p}^{-} \\
S_{p}^{+} & S_{p}^{-+}
\end{array}\right)
$$

holds for all $p \in P$ and such that the operators

$$
\Gamma_{p} \equiv\left(\begin{array}{cc}
\operatorname{pr}_{\operatorname{Ker} T_{0}} \circ\left(S_{p}\right)_{\left\lceil\operatorname{Coker} T_{0}\right.} & \operatorname{pr}_{\mathrm{Ker} T_{0}} \circ S_{p}^{-} \\
\left(S_{p}^{+}\right)_{\left\lceil\operatorname{Coker} T_{0}\right.} & S_{p}^{-+}
\end{array}\right): \operatorname{Coker} T_{0} \oplus Z^{-} \rightarrow \operatorname{Ker} T_{0} \oplus Z^{+}
$$

are invertible for all $p \in P$ and their inverses form a continuous family.

For the proof, we need the following basic result about the invertibility of block matrices in terms of the Schur complement.

Lemma 2.5 Let $A_{11}: X_{1} \rightarrow Y_{1}, A_{12}: X_{2} \rightarrow Y_{1}, A_{21}: X_{1} \rightarrow Y_{2}$ and $A_{22}: X_{2} \rightarrow Y_{2}$ be continuous linear maps between locally convex spaces such that

$$
\left(\begin{array}{ll}
A_{11} & A_{12} \\
A_{21} & A_{22}
\end{array}\right)^{-1}=\left(\begin{array}{ll}
B_{11} & B_{12} \\
B_{21} & B_{22}
\end{array}\right)
$$

for continuous linear maps $B_{i j}$ for $i, j=1,2$.

1. If $B_{22}$ is a topological isomorphism, then so is $A_{11}$, and its inverse is given by

$$
A_{11}^{-1}=B_{11}-B_{12} B_{22}^{-1} B_{21} \text {. }
$$

2. If $B_{22}=0$, then $A_{11} B_{11}$ and $B_{11} A_{11}$ are idempotent and satisfy

$$
\operatorname{Im}\left(A_{11} B_{11}\right)=\operatorname{Im} A_{11}, \quad \operatorname{Ker}\left(B_{11} A_{11}\right)=\operatorname{Ker} A_{11} .
$$

Proof The first statement is [75, Lemma 3.1] and can be verified by a direct calculation. The second statement follows from the identity

$$
A_{11} B_{11} A_{11}=A_{11}\left(\mathrm{id}_{X_{1}}-B_{12} A_{21}\right)=A_{11},
$$

where we used $B_{11} A_{11}+B_{12} A_{21}=\mathrm{id}_{X_{1}}$ and $A_{11} B_{12}=0$.

Proof of Theorem 2.4 First, suppose that $T$ is a uniformly regular family of linear maps. Then, by definition, we have topological decompositions $X=\operatorname{Ker} T_{0} \oplus \operatorname{Coim} T_{0}$, $Y=\operatorname{Coker} T_{0} \oplus \operatorname{Im} T_{0}$ and, for every $p \in P$, the map

$$
\tilde{T}_{p}=\operatorname{pr}_{\operatorname{Im} T_{0}} \circ\left(T_{p}\right)_{\uparrow \operatorname{Coim} T_{0}}
$$


is a topological isomorphism. Set $Z^{+}=\operatorname{Coker} T_{0}$ and $Z^{-}=\operatorname{Ker} T_{0}$, and let $T^{+}: Z^{+} \rightarrow Y$ and $T^{-}: X \rightarrow Z^{-}$be the canonical inclusion and projection, respectively. Moreover, let $S_{p}:=\tilde{T}_{p}^{-1} \circ \operatorname{pr}_{\operatorname{Im} T_{0}}: Y \rightarrow X$, and define $S_{p}^{-+}: Z^{-} \rightarrow Z^{+}$by

$$
S_{p}^{-+}=\operatorname{pr}_{\operatorname{Coker} T_{0}} \circ\left(T_{p} \circ \tilde{T}_{p}^{-1} \circ \operatorname{pr}_{\operatorname{Im} T_{0}}-\operatorname{id}_{Y}\right) \circ\left(T_{p}\right)_{\left\lceil\operatorname{Ker} T_{0}\right.} .
$$

Finally, define $S_{p}^{ \pm}$by

$$
\begin{gathered}
S_{p}^{+}=\operatorname{pr}_{\operatorname{Coker} T_{0}} \circ\left(\operatorname{id}_{Y}-T_{p} \circ S_{p}\right): Y \rightarrow Z^{+}, \\
S_{p}^{-}=\left(\operatorname{id}_{X}-S_{p} \circ T_{p}\right)_{\uparrow \operatorname{Ker} T_{0}}: Z^{-} \rightarrow X .
\end{gathered}
$$

Since, by definition, the inverses $\tilde{T}_{p}^{-1}$ form a continuous family $P \times \operatorname{Im} T_{0} \rightarrow \operatorname{Coim} T_{0}$, the families $S, S^{ \pm}, S^{-+}$are continuous. Furthermore, a direct calculation yields

$$
\begin{aligned}
& T_{p} \circ S_{p}=\operatorname{pr}_{\operatorname{Im} T_{0}}+\operatorname{pr}_{\operatorname{Coker} T_{0}} \circ T_{p} \circ \tilde{T}_{p}^{-1} \circ \operatorname{pr}_{\operatorname{Im} T_{0}}, \\
& S_{p} \circ T_{p}=\operatorname{pr}_{\operatorname{Coim} T_{0}}+\tilde{T}_{p}^{-1} \circ \operatorname{pr}_{\operatorname{Im} T_{0}} \circ\left(T_{p}\right)_{\left\lceil\operatorname{Ker} T_{0}\right.} .
\end{aligned}
$$

Using these identities, it is straightforward to check that (2.4) holds for every $p \in P$. Moreover, we have

$$
\Gamma_{p}=\left(\begin{array}{cc}
\operatorname{pr}_{\operatorname{Ker} T_{0}} \circ\left(S_{p}\right)_{\left\lceil\operatorname{Coker} T_{0}\right.} & \operatorname{pr}_{\operatorname{Ker} T_{0}} \circ S_{p}^{-} \\
\left(S_{p}^{+}\right)_{\left\lceil\operatorname{Coker} T_{0}\right.} & S_{p}^{-+}
\end{array}\right)=\left(\begin{array}{cc}
0 & \operatorname{id}_{\operatorname{Ker} T_{0}} \\
\operatorname{id}_{\operatorname{Coker} T_{0}} & S_{p}^{-+}
\end{array}\right),
$$

which is clearly invertible with a continuous family of inverses given by

$$
\Gamma_{p}^{-1}=\left(\begin{array}{cc}
-S_{p}^{-+} & \operatorname{id}_{\text {Coker } T_{0}} \\
\operatorname{id}_{\operatorname{Ker} T_{0}} & 0
\end{array}\right) .
$$

Conversely, let $T^{ \pm}, S, S^{ \pm}$and $S^{-+}$satisfying the assumptions of the second statement of Theorem 2.4. Since $S_{0}^{-+}=0$, Lemma 2.5(2) implies that $T_{0} \circ S_{0}$ and $S_{0} \circ T_{0}$ are idempotent with $\operatorname{Im} T_{0} \circ S_{0}=\operatorname{Im} T_{0}$ and $\operatorname{Ker} S_{0} \circ T_{0}=\operatorname{Ker} T_{0}$. Hence, $\operatorname{Ker} T_{0}$ and $\operatorname{Im} T_{0}$ are images of continuous idempotent operators, and as such they are closed and topologically complemented according to [45, Proposition 15.8.1]. As above, denote the complements by Coim $T_{0}$ and Coker $T_{0}$, respectively. It remains to show that $\tilde{T}_{p}=\operatorname{pr}_{\operatorname{Im} T_{0}} \circ\left(T_{p}\right)_{\uparrow \operatorname{Coim} T_{0}}$ is a topological isomorphism for all $p \in P$, and that $\tilde{T}_{p}^{-1}$ form a continuous family. For this purpose, we write all operators in block form with respect to the decompositions $X=\operatorname{Coim} T_{0} \oplus \operatorname{Ker} T_{0}$ and $Y=\operatorname{Im} T_{0} \oplus \operatorname{Coker} T_{0}$ (note the different order of the summands). Using this convention, the identity (2.4) becomes

$$
\begin{gathered}
\left(\begin{array}{ccc}
\tilde{T}_{p} & \operatorname{pr}_{\operatorname{Im} T_{0}} \circ\left(T_{p}\right)_{\left\lceil\operatorname{Ker} T_{0}\right.} & \operatorname{pr}_{\operatorname{Im} T_{0}} \circ T^{+} \\
\operatorname{pr}_{\operatorname{Coker} T_{0}} \circ\left(T_{p}\right)_{\left\lceil\operatorname{Coim} T_{0}\right.} & \operatorname{pr}_{\operatorname{Coker} T_{0}} \circ\left(T_{p}\right)_{\left\lceil\operatorname{Ker} T_{0}\right.} & \operatorname{pr}_{\operatorname{Coker} T_{0}} \circ T^{+} \\
\left(T^{-}\right)_{\left\lceil\operatorname{Coim} T_{0}\right.} & \left(T^{-}\right)_{\left\lceil\operatorname{Ker} T_{0}\right.} & 0
\end{array}\right)^{-1} \\
=\left(\begin{array}{ccc}
\operatorname{pr}_{\operatorname{Coim} T_{0}} \circ\left(S_{p}\right)_{\left\lceil\operatorname{Im} T_{0}\right.} & \operatorname{pr}_{\operatorname{Coim} T_{0}} \circ\left(S_{p}\right)_{\left\lceil\operatorname{Coker} T_{0}\right.} & \operatorname{pr}_{\operatorname{Coim} T_{0}} \circ S_{p}^{-} \\
\operatorname{pr}_{\operatorname{Ker} T_{0}} \circ\left(S_{p}\right)_{\left\lceil I m T_{0}\right.} & \operatorname{pr}_{\operatorname{Ker} T_{0}} \circ\left(S_{p}\right)_{\left\lceil\operatorname{Coker} T_{0}\right.} & \operatorname{pr}_{\operatorname{Ker} T_{0}} \circ S_{p}^{-} \\
\left(S_{p}^{+}\right)_{\left\lceil\operatorname{Im} T_{0}\right.} & \left(S_{p}^{+}\right)_{\left\lceil\operatorname{Coker} T_{0}\right.} & S_{p}^{-+}
\end{array}\right) .
\end{gathered}
$$


These matrices should be read as operators from $\operatorname{Im} T_{0} \oplus \operatorname{Coker} T_{0} \oplus Z^{-}$to Coim $T_{0} \oplus \operatorname{Ker} T_{0} \oplus Z^{+}$. Note that the lower right two-times-two block of the right-hand side coincides with the operator $\Gamma_{p}$. Since $\Gamma_{p}$ is invertible by assumption, Lemma 2.5(1) shows that $\tilde{T}_{p}$ is invertible, too. Moreover, the inverses are given by

$$
\begin{aligned}
\tilde{T}_{p}^{-1}= & \operatorname{pr}_{\operatorname{Coim} T_{0}} \circ\left(S_{p}\right)_{\left\lceil\operatorname{Im} T_{0}\right.} \\
& -\left(\begin{array}{c}
\operatorname{pr}_{\operatorname{Coim} T_{0}} \circ\left(S_{p}\right)_{\left\lceil\operatorname{Coker} T_{0}\right.} \\
\operatorname{pr}_{\operatorname{Coim} T_{0}} \circ S_{p}^{-}
\end{array}\right) \Gamma_{p}^{-1}\left(\begin{array}{c}
\operatorname{pr}{\operatorname{Ker} T_{0}}_{p} \circ\left(S_{p}\right)_{\left\lceil\operatorname{Im} T_{0}\right.} \\
\left(S_{p}^{+}\right)_{\left\lceil\operatorname{Im} T_{0}\right.}
\end{array}\right)
\end{aligned}
$$

and thus form a continuous family $P \times \operatorname{Im} T_{0} \rightarrow$ Coim $T_{0}$. Hence, $T$ is uniformly regular.

For the special case of a single operator, we obtain the following.

Corollary 2.6 A continuous linear map $T: X \rightarrow Y$ between locally convex spaces is regular if and only if there exist locally convex spaces $Z^{ \pm}$, continuous linear maps $T^{+}: Z^{+} \rightarrow Y$, $T^{-}: X \rightarrow Z^{-}$and $S: Y \rightarrow X, S^{-}: Z^{-} \rightarrow X, S^{+}: Y \rightarrow Z^{+}$such that

$$
\left(\begin{array}{cc}
T & T^{+} \\
T^{-} & 0
\end{array}\right)^{-1}=\left(\begin{array}{cc}
S & S^{-} \\
S^{+} & 0
\end{array}\right) .
$$

Remark 2.7 In the setting of Corollary 2.6, it is straightforward to verify that $T \circ S \circ T=T$ holds. An operator $S$ satisfying such a relation is called a generalized inverse of $T$, cf. [56, $64,66]$. In fact, one can show that regularity of an operator is equivalent to the existence of a generalized inverse. Since we do not need this point of view in the remainder, we refer to $[13,36]$ for further details.

Remark 2.8 (Uniform regularity in the tame Fréchet category) It is clear that a version of Theorem 2.4 holds in the tame Fréchet category if the word "tame" is inserted in the right places (see Appendix A for a brief overview of the main concepts of tame Fréchet spaces). Let us spell out the details.

Let $X$ and $Y$ be tame Fréchet spaces and let $T: P \times X \rightarrow Y$ be a tame smooth family of linear maps. Then, $T$ is called uniformly tame regular if there exist tame decompositions $X=\operatorname{Ker} T_{0} \oplus \operatorname{Coim} T_{0}$ and $Y=\operatorname{Coker} T_{0} \oplus \operatorname{Im} T_{0}$, and, for every $p \in P$, the map $\tilde{T}_{p}=\operatorname{pr}_{\operatorname{Im} T_{0}} \circ\left(T_{p}\right)_{\left\lceil\operatorname{Coim} T_{0}\right.}: \operatorname{Coim} T_{0} \rightarrow \operatorname{Im} T_{0}$ is a tame isomorphism such that the inverses form a tame smooth family $P \times \operatorname{Im} T_{0} \rightarrow \operatorname{Coim} T_{0}$. Then, the equivalence of Theorem 2.4 holds with $Z^{ \pm}$being tame Fréchet spaces, $T^{ \pm}$being tame maps and $S, S^{ \pm}, S^{-+}, \Gamma^{-1}$ being tame smooth families.

\subsection{Fredholm operators}

An important class of examples of regular operators is given by Fredholm operators. Fredholm operators are usually studied as maps between Banach spaces (or Hilbert spaces), but most results extend to the locally convex setting, cf. [22, 71, 72]. 
Definition 2.9 A continuous linear map $T: X \rightarrow Y$ between locally convex spaces is called a Fredholm operator if $T$ is relatively open, the kernel of $T$ is a finite-dimensional subspace of $X$, and the image of $T$ is a finite-codimensional closed subspace of $Y$. The index ind $T$ of a Fredholm operator $T$ is defined by

$$
\text { ind } T=\operatorname{dim} \operatorname{Ker} T-\operatorname{dim} \operatorname{Coker} T \text {. }
$$

Since finite-dimensional subspaces and finite-codimensional closed subspaces of a locally convex space are always topologically complemented according to [45, Propositions 15.8.2 and 20.5.5], every Fredholm operator is regular.

For a continuous family $T$ of linear maps with $T_{0}$ being a Fredholm operator, the invertibility of the family $\Gamma$ in Theorem 2.4 is automatic.

Corollary 2.10 Let $T: P \times X \rightarrow Y$ be a continuous family of linear maps between locally convex spaces $X$ and $Y$ such that $T_{0}$ is a Fredholm operator. Then, $T$ is uniformly regular if and only if there exist finite-dimensional spaces $Z^{ \pm}$and continuous linear maps $T^{+}: Z^{+} \rightarrow Y$ and $T^{-}: X \rightarrow Z^{-}$such that, after possibly shrinking $P$,

$$
\left(\begin{array}{cc}
T_{p} & T^{+} \\
T^{-} & 0
\end{array}\right)^{-1}=\left(\begin{array}{cc}
S_{p} & S_{p}^{-} \\
S_{p}^{+} & S_{p}^{-+}
\end{array}\right)
$$

holds for all $p \in P$, where $S: P \times Y \rightarrow X, S^{-}: P \times Z^{-} \rightarrow X, S^{+}: P \times Y \rightarrow Z^{+}$and $S^{-+}: P \times Z^{-} \rightarrow Z^{+}$are continuous families of linear maps with $S_{0}^{-+}=0$.

Proof If $T$ is uniformly regular, then the proof of Theorem 2.4 shows that one can choose $Z^{+}=\operatorname{Coker} T_{0}$ and $Z^{-}=\operatorname{Ker} T_{0}$. Both spaces are finite-dimensional, because $T_{0}$ is a Fredholm operator. This establishes one direction.

Conversely, let $T^{ \pm}, S, S^{ \pm}, S^{-+}$be given as stated above. By Theorem 2.4, it suffices to show that the operator

$$
\Gamma_{p}=\left(\begin{array}{cc}
\operatorname{pr}_{\operatorname{Ker} T_{0}} \circ\left(S_{p}\right)_{\left\lceil\operatorname{Coker} T_{0}\right.} & \operatorname{pr}_{\operatorname{Ker} T_{0}} \circ S_{p}^{-} \\
\left(S_{p}^{+}\right)_{\left\lceil\operatorname{Coker} T_{0}\right.} & S_{p}^{-+}
\end{array}\right): \text {Coker } T_{0} \oplus Z^{-} \rightarrow \operatorname{Ker} T_{0} \oplus Z^{+}
$$

is invertible and that the inverses form a continuous family. Since $S_{0}^{-+}=0$, Lemma 2.5(2) implies $S_{0}^{-} \circ T^{-}=\operatorname{pr}_{\operatorname{Ker} T_{0}}$ and $T^{+} \circ S_{0}^{+}=\operatorname{pr}_{\operatorname{Coker} T_{0}}$. A straightforward calculation using these identities shows that we have

$$
\Gamma_{0}=\left(\begin{array}{cc}
0 & \operatorname{pr}_{\mathrm{Ker} T_{0}} \circ S_{0}^{-} \\
\left(S_{0}^{+}\right)_{\left\lceil\operatorname{Coker} T_{0}\right.} & 0
\end{array}\right)
$$

and

$$
\Gamma_{0}^{-1}=\left(\begin{array}{cc}
0 & \operatorname{pr}_{\operatorname{Coker} T_{0}} \circ T^{+} \\
\left(T^{-}\right)_{\left\lceil\operatorname{Ker} T_{0}\right.} & 0
\end{array}\right)
$$

Since, for every $p \in P, \Gamma_{p}$ is an operator between finite-dimensional spaces and $\Gamma_{0}$ is invertible, we can shrink $P$ in such a way that $\Gamma_{p}$ is invertible for all $p \in P$ and that the inverses form a continuous family. Thus, Theorem 2.4 implies that $T$ is uniformly regular. 
In the Banach setting, the set of Fredholm operators is open in the space of bounded linear operators and the index does not change under a continuous deformation [42, Corollary 19.1.6]. This statement relies on the openness of the set of invertible operators and thus does not carry over to the locally convex setting. The following proposition shows that the notion of uniform regularity is an adequate substitute.

Proposition 2.11 Let $T: P \times X \rightarrow Y$ be a uniformly regular family of linear maps between locally convex spaces $X$ and $Y$. If $T_{0}$ is Fredholm, then $T_{p}$ is Fredholm and ind $T_{p}=\operatorname{ind} T_{0}$ for all $p \in P$.

Proof By Corollary 2.10, there exist finite-dimensional spaces $Z^{ \pm}$and continuous linear maps $T^{+}: Z^{+} \rightarrow Y$ and $T^{-}: X \rightarrow Z^{-}$such that

$$
\left(\begin{array}{cc}
T_{p} & T^{+} \\
T^{-} & 0
\end{array}\right)
$$

is invertible for all $p \in P$. This is only possible if $\operatorname{Ker} T_{p}$ and $\operatorname{Coker} T_{p}$ are finite-dimensional so that $T_{p}$ has to be Fredholm. Moreover, using the invariance of the index under finite-rank perturbations, we have

$$
0=\operatorname{ind}\left(\begin{array}{cc}
T_{p} & T^{+} \\
T^{-} & 0
\end{array}\right)=\operatorname{ind} T_{p}+\operatorname{dim} Z^{+}-\operatorname{dim} Z^{-}=\operatorname{ind} T_{p}-\operatorname{ind} T_{0},
$$

which establishes the formula for the index.

As we discuss now, families of elliptic operators constitute an important class of examples of uniformly regular Fredholm operators. Let $E \rightarrow M$ and $F \rightarrow M$ be finite-dimensional vector bundles over a compact manifold $M$ without boundary. Endow the spaces $\mathcal{E}$ and $\mathcal{F}$ of smooth sections of $E$ and $F$, respectively, with the compact-open $C^{\infty}$-topology. With respect to this topology, these section spaces are tame Fréchet spaces, see [35, Theorem II.2.3.1]. A continuous linear map $L: \mathcal{E} \rightarrow \mathcal{F}$ is a partial differential operator of degree $r$ if and only if there exists a vertical vector bundle morphism $f: \mathrm{J}^{r} E \rightarrow F$ such that $L$ factors through the jet bundle $\mathrm{J}^{r} E$ as follows:

$$
L: \mathcal{E} \stackrel{\mathrm{j}^{r}}{\longrightarrow} \Gamma^{\infty}\left(\mathrm{J}^{r} E\right) \stackrel{f_{*}}{\longrightarrow} \mathcal{F}
$$

where $\mathrm{j}^{r}$ denotes the $r$-th jet prolongation and $f_{*}$ is the push-forward by $f$. We refer to $f$ as the coefficients of $L$ and will sometimes write $L_{f}$ instead of $L$ to emphasize this relation. Recall that the principal symbol $\sigma_{f}$ of $L_{f}$ is a homogeneous polynomial of degree $r$ on $\mathrm{T}^{*} M$ with values in the bundle $\mathrm{L}(E, F)$ of fiberwise linear maps $E \rightarrow F$. A differential operator $L_{f}$ with coefficients $f$ is called elliptic if its symbol is invertible; that is, for each nonzero $p \in \mathrm{T}^{*} M$, the bundle map $\sigma_{f}(p, \ldots, p) \in \mathrm{L}(E, F)$ is invertible.

It is a standard result in elliptic theory that every elliptic differential operator over a compact manifold is a Fredholm operator between appropriate Sobolev spaces [42, Theorem 19.2.1]. The same holds true in the tame Fréchet category. In fact, more is true: elliptic operators are regularly parametrized by their coefficients.

Theorem 2.12 Let $E \rightarrow M$ and $F \rightarrow M$ be finite-dimensional vector bundles over a compact manifold $M$ without boundary, and denote the space of smooth sections of $E$ and $F$ by 
$\mathcal{E}$ and $\mathcal{F}$, respectively. The parametrization of a partial differential operator by their coefficients yields a tame smooth family

$$
L: \Gamma^{\infty}\left(\mathrm{L}\left(\mathrm{J}^{r} E, F\right)\right) \times \mathcal{E} \rightarrow \mathcal{F}, \quad(f, \phi) \mapsto L_{f}(\phi)
$$

of linear operators which is uniformly tame regular in a neighborhood of every $f_{0} \in \Gamma^{\infty}\left(\mathrm{L}\left(\mathrm{J}^{r} E, F\right)\right)$ for which $L_{f_{0}}$ is an elliptic differential operator.

Proof Let $f_{0} \in \Gamma^{\infty}\left(\mathrm{L}\left(\mathbf{J}^{r} E, F\right)\right)$ be such that $L_{f_{0}}$ is an elliptic differential operator. By [35, Theorem II.3.3.3], there exist an open neighborhood $\mathcal{U}$ of $f_{0}$ in $\Gamma^{\infty}\left(\mathrm{L}\left(\mathrm{J}^{r} E, F\right)\right)$, finitedimensional vector spaces $Z^{ \pm}$and continuous linear maps $L^{+}: Z^{+} \rightarrow Y$ and $L^{-}: X \rightarrow Z^{-}$ such that

$$
\left(\begin{array}{cc}
L_{f} & L^{+} \\
L^{-} & 0
\end{array}\right): \mathcal{E} \times Z^{+} \rightarrow \mathcal{F} \times Z^{-}
$$

is invertible for all $f \in \mathcal{U}$. Moreover, the inverses form a tame smooth family $\mathcal{U} \times \mathcal{F} \times Z^{-} \rightarrow \mathcal{E} \times Z^{+}$of linear operators. Hence, by Corollary $2.10, L_{\uparrow \mathcal{U} \times \mathcal{E}}$ is uniformly tame regular at $f_{0}$.

\subsection{Elliptic complexes}

In this section, the notion of uniform regularity is extended to linear chain complexes. The main application we have in mind is elliptic complexes.

Let $P$ be a neighborhood of 0 in some locally convex space, let $X_{i}$ be a sequence of locally convex spaces and let $T_{i}: P \times X_{i} \rightarrow X_{i+1}$ be a sequence of continuous families of linear maps such that $\left(X_{i}, T_{i, 0}\right)$ is a complex, i.e., $T_{i+1,0} \circ T_{i, 0}=0$ for all $i \in \mathbb{Z}$. We say that $\left(P, X_{i}, T_{i}\right)$ is a continuous family of chains. Simple examples (cf. Example 2.16 below) show that a deformation of a chain complex is in general not a complex; this is why we require $T_{i, p}$ to form a complex only at $p=0$. The following notion is a natural generalization of uniform regularity to chains.

Definition 2.13 A continuous family of chains $\left(P, X_{i}, T_{i}\right)$ is called uniformly regular (at 0$)$ if the following holds for every $i \in \mathbb{Z}$ :

1. The image of $T_{i-1,0}$ is closed in $X_{i}$, and there exist closed subspaces $H_{i}$ and Coim $T_{i, 0}$ of $X_{i}$ such that

$$
X_{i}=\operatorname{Im} T_{i-1,0} \oplus \operatorname{Coim} T_{i, 0} \oplus H_{i}
$$

is a topological decomposition and $H_{i} \subseteq \operatorname{Ker} T_{i, 0}$.

2. For every $p \in P$, the map

$$
\tilde{T}_{i, p}=\operatorname{pr}_{\operatorname{Im} T_{i, 0}} \circ\left(T_{i, p}\right)_{\uparrow \operatorname{Coim} T_{i, 0}}: \operatorname{Coim} T_{i, 0} \rightarrow \operatorname{Im} T_{i, 0}
$$

is a topological isomorphism such that the inverses form a continuous family $P \times \operatorname{Im} T_{i, 0} \rightarrow \operatorname{Coim} T_{i, 0}$. 
If additionally, for every $i \in \mathbb{Z}, X_{i}$ is a tame Fréchet space, $T_{i}$ is a tame smooth family, the decomposition (2.29) of $X_{i}$ is tame and $\tilde{T}_{i, p}$ are a tame isomorphisms such that the inverses form a tame smooth family, then $\left(P, X_{i}, T_{i}\right)$ is called uniformly tame regular.

By definition, for an uniformly regular family $\left(P, X_{i}, T_{i}\right)$ of chains, we have

$$
\operatorname{Ker} T_{i, 0}=\operatorname{Im} T_{i-1,0} \oplus H_{i},
$$

which justifies the notion Coim $T_{i, 0}$ for the subspace in the decomposition (2.29). The subspaces $H_{i}$ are identified with the homology groups for the complex at $p=0$, that is,

$$
H_{i} \simeq \operatorname{Ker} T_{i, 0} / \operatorname{Im} T_{i-1,0} .
$$

For the applications we have in mind, the following characterization of uniform regularity of chains turns out to be more convenient. It entails that, roughly speaking, a family of chains $\left(P, X_{i}, T_{i}\right)$ is uniformly regular if each family $T_{i}$ of linear maps is uniformly regular after factoring-out the image of the direct predecessor $T_{i-1,0}$.

Proposition 2.14 A continuous family of chains $\left(P, X_{i}, T_{i}\right)$ is uniformly regular if and only if, for every $i \in \mathbb{Z}$, the subspace $\operatorname{Im} T_{i-1,0}$ of $X_{i}$ is closed and topologically complemented, say $X_{i}=\operatorname{Im} T_{i-1,0} \oplus \operatorname{Coker} T_{i-1,0}$, and the continuous family $p \mapsto\left(T_{i, p}\right)_{\left\lceil\operatorname{Coker} T_{i-1,0}\right.}$ of linear maps is uniformly regular.

Proof The claim is a simple consequence of the observation that the image of $\left(T_{i, 0}\right)_{\left\lceil\operatorname{Coker} T_{i-1,0}\right.}$ coincides with the image of $T_{i, 0}$ and that

$$
H_{i} \simeq \operatorname{Ker}\left(T_{i, 0}\right)_{\lceil\operatorname{Coker}} T_{i-1,0}
$$

holds, because $T_{i, 0}$ is a complex.

Let us now turn to deformations of elliptic complexes. Let $E_{0}, E_{1}, \ldots, E_{N}$ be a sequence of finite-dimensional vector bundles over a compact manifold $M$, and let $\mathcal{E}_{i}$ be the tame Fréchet space of smooth sections of $E_{i}$. Moreover, let $P$ be an open neighborhood of 0 in some tame Fréchet space and let $L_{i}: P \times \mathcal{E}_{i} \rightarrow \mathcal{E}_{i+1}$ be a sequence of differential operators parametrized by points of $P$. We assume that, for every $i \in \mathbb{Z}$, the parametrization factors through the space of coefficients as follows:

$$
P \times \mathcal{E}_{i} \stackrel{\hat{L}_{i} \times \mathrm{id}_{\mathcal{E}_{i}}}{\longrightarrow} \Gamma^{\infty}\left(\mathrm{L}\left(\mathrm{J}^{r_{i}} E_{i}, E_{i+1}\right)\right) \times \mathcal{E}_{i} \longrightarrow \mathcal{E}_{i+1}
$$

where $\hat{L}_{i}: P \rightarrow \Gamma^{\infty}\left(\mathrm{L}\left(\mathrm{J}^{r_{i}} E_{i}, E_{i+1}\right)\right)$ is a tame smooth map and the second map is the parametrization of differential operators by their coefficients as defined in (2.27). For simplicity, let us assume that the degree $r_{i}$ of the differential operator $L_{i, p}: \mathcal{E}_{i} \rightarrow \mathcal{E}_{i+1}$ is the same for all $p \in P$ and $i \in \mathbb{Z}$. We will refer to this setting by saying that $\left(P, \mathcal{E}_{i}, L_{i}\right)$ is a tame family of chains of differential operators. A chain complex $L_{i}: \mathcal{E}_{i} \rightarrow \mathcal{E}_{i+1}$ of differential operators is called elliptic if the sequence of principal symbols

$$
\cdots \longrightarrow \hbar^{*} E_{i} \stackrel{\sigma\left(L_{i}\right)}{\longrightarrow} \AA^{*} E_{i+1} \longrightarrow \cdots
$$

is exact outside of the zero section of the cotangent bundle $\stackrel{\star}{\tau}: \mathrm{T}^{*} M \rightarrow M$. 
As a generalization of Theorem 2.12, we have the following result concerning deformations of elliptic complexes.

Theorem 2.15 Let $E_{0}, \ldots, E_{N}$ be a sequence of finite-dimensional vector bundles over a compact manifold $M$, and denote the space of smooth sections of $E_{i}$ by $\mathcal{E}_{i}$. Let $\left(P, \mathcal{E}_{i}, L_{i}\right)$ be a tame family of chains of differential operators. If $\left(\mathcal{E}_{i}, L_{i, 0}\right)$ is an elliptic complex, then $\left(P, \mathcal{E}_{i}, L_{i}\right)$ is uniformly tame regular (after possibly shrinking $P$ ).

Proof The proof is inspired by the proof of [3, Proposition 6.1], where a parametrix of an elliptic complex is constructed by using the parametrix of an elliptic operator. Similarly, we will reduce the question of the uniform tame regularity of the chain to the uniform regularity of a deformation of differential operators, for which we can employ Theorem 2.12.

For this purpose, fix a Riemannian metric on $M$ and a fiber Riemannian metric on every vector bundle $E_{i}$. These data define a natural $\mathrm{L}^{2}$-inner product on $\mathcal{E}_{i}$. By partial integration, we see that the adjoints $L_{i, p}^{*}: \mathcal{E}_{i+1} \rightarrow \mathcal{E}_{i}$ of $L_{i, p}$ with respect to these inner products yield a tame family of chains of differential operators. For every $i \in \mathbb{Z}$, define the tame family $\Delta_{i}: P \times \mathcal{E}_{i} \rightarrow \mathcal{E}_{i}$ by

$$
\Delta_{i, p}=L_{i, 0}^{*} \circ L_{i, p}+L_{i-1, p} \circ L_{i-1,0}^{*} .
$$

Clearly, $\Delta_{i}$ is a family of differential operators of order $2 r$. Moreover, $\Delta_{i, 0}$ is an elliptic operator, because $\left(\mathcal{E}_{i}, L_{i, 0}\right)$ is an elliptic complex by assumption. Thus, Theorem 2.12 implies that the family $\Delta_{i}$ is uniformly tame regular. In particular, $\Delta_{i, 0}$ is regular and self-adjoint so that we get the following topological decomposition

$$
\mathcal{E}_{i}=\operatorname{Ker} \Delta_{i, 0} \oplus \operatorname{Im} \Delta_{i, 0} .
$$

Moreover, $\tilde{\Delta}_{i, p}=\operatorname{pr}_{\operatorname{Im} \Delta_{i, 0}} \circ\left(\Delta_{i, p}\right)_{\mid \operatorname{Im} \Delta_{i, 0}}$ is a tame automorphism of $\operatorname{Im} \Delta_{i, 0}$ for every $p \in P$ (after possibly shrinking $P$ ) in such a way that the inverses form a tame smooth family. The decomposition (2.37) implies that the images of $L_{i-1,0}$ and $L_{i, 0}^{*}$ are closed and that they fit into the topological decomposition

$$
\mathcal{E}_{i}=\operatorname{Im} L_{i-1,0} \oplus \operatorname{Im} L_{i, 0}^{*} \oplus H_{i},
$$

where $H_{i} \equiv \operatorname{Ker} \Delta_{i, 0}=\operatorname{Ker} L_{i, 0} \cap \operatorname{Ker} L_{i-1,0}^{*}$. Finally, a direct calculation shows that, for every $i \in \mathbb{Z}$, the tame smooth family $G_{i}$ defined by

$$
G_{i, p}=L_{i, 0}^{*} \circ\left(\tilde{\Delta}_{i+1, p}^{-1}\right)_{\left\lceil\operatorname{Im} L_{i, 0}\right.}: \operatorname{Im} L_{i, 0} \rightarrow \operatorname{Im} L_{i, 0}^{*}
$$

is an inverse of the family

$$
\tilde{L}_{i, p}=\operatorname{pr}_{\operatorname{Im} L_{i, 0}} \circ\left(L_{i, p}\right)_{\left\lceil\operatorname{Im} L_{i, 0}^{*}\right.}: \operatorname{Im} L_{i, 0}^{*} \rightarrow \operatorname{Im} L_{i, 0} .
$$

This shows that $\left(P, \mathcal{E}_{i}, L_{i}\right)$ is uniformly tame regular.

Example 2.16 Let $P \rightarrow M$ be a finite-dimensional principal $G$-bundle over a compact manifold $M$, and let $E$ be an associated vector bundle. The space $\mathcal{C}(P)$ of connections on $P$ is an affine tame Fréchet space. Every connection $A \in \mathcal{C}(P)$ yields via the covariant exterior differential on $E$-valued forms a chain 


$$
\cdots \longrightarrow \Omega^{i}(M, E) \stackrel{\mathrm{d}_{A}}{\longrightarrow} \Omega^{i+1}(M, E) \longrightarrow \cdots
$$

As this chain is an elliptic complex if the connection is flat, Theorem 2.15 entails that the family of chains $\left(\mathcal{C}(P), \Omega^{k}(M, E), \mathrm{d}_{A}\right)$ is uniformly tame regular in a neighborhood of every flat connection $A_{0} \in \mathcal{C}(P)$. Moreover, by Proposition 2.14, the family

$$
\mathcal{C}(P) \times \Omega^{0}(M, E) \rightarrow \Omega^{1}(M, E), \quad(A, \alpha) \mapsto \mathrm{d}_{A} \alpha
$$

is uniformly regular at every $A_{0} \in \mathcal{C}(P)$ (we do not need flatness of $A_{0}$ for this in 0-degree). The operator defined in (2.36) takes here the following form

$$
\Delta_{A_{0} A}=\mathrm{d}_{A_{0}}^{*} \mathrm{~d}_{A}+\mathrm{d}_{A}^{*} \mathrm{~d}_{A_{0}}: \Omega^{k}(M, E) \rightarrow \Omega^{k}(M, E)
$$

and is a natural extension of the Faddeev-Popov operator to forms of higher degree, cf. [70, eq. (8.4.8)]. A similar operator played a central role in [15, p. 405] for the study of the curvature map $F: \mathcal{C}(P) \rightarrow \Omega^{2}(M, \operatorname{Ad} P)$ near a flat connection.

\section{Normal form of a nonlinear map}

In this section, we study the local behavior of a smooth map $f: M \rightarrow N$ between (infinitedimensional) manifolds. We introduce the concept of a normal form and find suitable conditions that ensure that $f$ can be brought into such a normal form.

In the linear setting, we have seen that every regular operator factorizes through a linear isomorphism. Similarly, every nonlinear map can be represented locally by a linear isomorphism up to some higher-order error term. In fact, for a given smooth map $f: M \rightarrow N$ and $m \in M$, consider its local representative $\tilde{f}:=\rho \circ f \circ \kappa^{-1}: X \supseteq U \rightarrow Y$ with respect to charts $^{3} \kappa: M \supseteq U^{\prime} \rightarrow U \subseteq X$ at $m$ and $\rho: N \supseteq V^{\prime} \rightarrow V \subseteq Y$ at $f(m)$ modeling $M$ on $X \simeq \mathrm{T}_{m} M$ and $N$ on $Y \simeq \mathrm{T}_{f(m)} N$, respectively. Suppose $\mathrm{T}_{0} \tilde{f}: X \rightarrow Y$ is a regular operator with decompositions $X=\operatorname{Ker}_{0} \tilde{f} \oplus \operatorname{Coim~}_{0} \tilde{f}, Y=\operatorname{Coker}_{0} \tilde{f} \oplus \operatorname{Im~T}_{0} \tilde{f}$ and core $\hat{f}: \operatorname{Coim~}_{0} \tilde{f} \rightarrow \operatorname{Im~T}_{0} \tilde{f}$. Then defining $f_{\text {sing }}:=\tilde{f}-\hat{f}: X \supseteq U \rightarrow Y$ we get the following local representation of $f$ :

$$
\rho \circ f \circ \kappa^{-1}=\hat{f}+f_{\text {sing }} .
$$

Thus, the singular part $f_{\text {sing }}$ obstructs $f$ from being locally represented by the isomorphism $\hat{f}$. The concrete form of $f_{\text {sing }}$ depends, of course, on the chosen charts. The aim is to construct charts such that the singular part satisfies additional properties, which are formalized in the following definition.

\footnotetext{
${ }^{3}$ Throughout this work, we follow the convention that a chart $\kappa: M \supseteq U^{\prime} \rightarrow U \subseteq X$ at a point $m \in M$ satisfies $\kappa(m)=0$. Moreover, $U^{\prime}$ and $U$ are understood to be open neighborhoods of $m$ in $M$ and of 0 in $X$, respectively.
} 
Definition 3.1 An abstract normal form consists of a tuple $\left(X, Y, \hat{f}, f_{\text {sing }}\right)$, where

1. $X$ and $Y$ are locally convex spaces with topological decompositions ${ }^{4} X=\operatorname{Ker} \oplus$ Coim and $Y=\operatorname{Coker} \oplus \mathrm{Im}$,

2. $\hat{f}:$ Coim $\rightarrow$ Im is a linear topological isomorphism,

3. $f_{\text {sing }}: X \supseteq U \rightarrow$ Coker is a smooth map defined on an open neighborhood $U$ of 0 in $X$ such that $f_{\text {sing }}\left(0, x_{2}\right)=0$ holds for all $x_{2} \in U \cap$ Coim and such that the derivative $\mathrm{T}_{(0,0)} f_{\text {sing }}: X \rightarrow$ Coker of $f_{\text {sing }}$ at $(0,0)$ vanishes.

Given an abstract normal form $\left(X, Y, \hat{f}, f_{\text {sing }}\right)$, set $f_{\mathrm{NF}}=\hat{f}+f_{\text {sing }}: X \supseteq U \rightarrow Y$.

A normal form $\left(X, Y, \hat{f}, f_{\text {sing }}\right)$ is called tame if $X, Y$ are tame Fréchet spaces with tame decompositions $X=\operatorname{Ker} \oplus$ Coim, $Y=$ Coker $\oplus \operatorname{Im}, \hat{f}$ is a tame isomorphism and $f_{\text {sing }}$ is a tame smooth map.

The 0 -level set of $f_{\mathrm{NF}}$ is given by

$$
f_{\mathrm{NF}}^{-1}(0)=\left\{\left(x_{1}, 0\right) \in U: f_{\text {sing }}\left(x_{1}, 0\right)=0\right\} .
$$

Since $\mathrm{T}_{(0,0)} f_{\text {sing }}=0$, the level set $f_{\mathrm{NF}}^{-1}(0)$ is in general not a smooth manifold. Its singular structure is completely determined by $f_{\text {sing }}$. For this reason, we refer to $f_{\text {sing }}$ as the singular part of $f_{\mathrm{NF}}$.

Definition 3.2 We say that a smooth map $f: M \rightarrow N$ between manifolds can be brought into the normal form $\left(X, Y, \hat{f}, f_{\text {sing }}\right)$ at the point $m \in M$ if there exist charts $\kappa: M \supseteq U^{\prime} \rightarrow U \subseteq X$ at $m$ and $\rho: N \supseteq V^{\prime} \rightarrow V \subseteq Y$ at $f(m)$ such that $f\left(U^{\prime}\right) \subseteq V^{\prime}, f_{\text {sing }}$ is defined on $U$ and

$$
\rho \circ f_{\uparrow U^{\prime}} \circ \kappa^{-1}=f_{\mathrm{NF}}
$$

holds. For short, we say that $f$ is locally equivalent to $f_{\mathrm{NF}}$.

Assume that the smooth map $f: M \rightarrow N$ can be brought into the normal form $\left(X, Y, \hat{f}, f_{\text {sing }}\right)$ at the point $m \in M$ using diffeomorphisms $\kappa: U^{\prime} \rightarrow U$ and $\rho: V^{\prime} \rightarrow V$. The isomorphisms $\mathrm{T}_{m} \kappa: \mathrm{T}_{m} M \rightarrow X$ and $\mathrm{T}_{f(m)} \rho: \mathrm{T}_{f(m)} N \rightarrow Y$ identify the abstract spaces $X$ and $Y$ with the tangent spaces of $M$ and $N$, respectively. Under these identifications, the spaces Ker and $\mathrm{Im}$ in the decomposition of $X$ and $Y$ coincide with the kernel and the image of $\mathrm{T}_{m} f$.

In certain cases, a normal form amounts to a linearization of the map under consideration. We say that a smooth map $f: M \rightarrow N$ is a submersion at $m \in M$ if it is equivalent to a linear projection in a neighborhood of $m$. Similarly, $f$ is called an immersion at $m$ if it is equivalent to a linear injection in a neighborhood of $m$. More generally, $f$ is a subimmersion at $m$ if it is equivalent to a linear map in a neighborhood of $m$. The following proposition connects these definitions to the perhaps more classical characterizations known from finite-dimensional geometry.

\footnotetext{
${ }^{4}$ In these decompositions Ker, Coim, etc., denote abstract spaces. Below, we will identify them with the kernel, coimage, etc., of the tangent map $\mathrm{T}_{m} f$, respectively.
} 
Proposition 3.3 Let $f: M \rightarrow N$ be a smooth map. Assume that $f$ can be brought into a normal form in a neighborhood $U^{\prime}$ of $m \in M$. Then, the following holds:

1. (Submersion) $f$ is a submersion at $m$ if and only if $\mathrm{T}_{m} f$ is surjective.

2. (Immersion) $f$ is an immersion at $m$ if and only if $\mathrm{T}_{m} f$ is injective.

3. (Constant rank) $f$ is a subimmersion at $m$ if $\mathrm{T}_{p} f$ is a finite-rank operator ${ }^{5}$ satisfying $\mathrm{rkT}_{p} f=\mathrm{rkT}_{m} f$ for all $p \in U^{\prime}$.

Proposition 3.3(3) generalizes to maps whose derivatives have a constant but not necessarily finite-dimensional image, cf. [51, Theorem 2.5.15] for a Banach version.

Proof As the claim is of local nature, it suffices to consider the case where $f=f_{\mathrm{NF}}=\hat{f}+f_{\text {sing }}$ for an abstract normal form $\left(X, Y, \hat{f}, f_{\text {sing }}\right)$, and $m=0$. If $\mathrm{T}_{0} f_{\mathrm{NF}}=\hat{f} \circ \mathrm{pr}_{\text {Coim }}$ is surjective, then Coker is trivial and hence $f_{\text {sing }}=0$. Similarly, if $\mathrm{T}_{0} f_{\mathrm{NF}}$ is injective, then Coim $=X$ and thus $f_{\text {sing }}=0$, because $f_{\text {sing }}\left(0, x_{2}\right)=0$ for all $x_{2} \in U \cap$ Coim by assumption. Moreover, we have

$$
\mathrm{T}_{x} f_{\mathrm{NF}}\left(v_{1}, v_{2}\right)=\left(\mathrm{T}_{x} f_{\text {sing }}\left(v_{1}, v_{2}\right), \hat{f}\left(v_{2}\right)\right) \in \operatorname{Coker} \oplus \operatorname{Im}=Y
$$

for all $x \in U, \quad v_{1} \in$ Ker and $v_{2} \in$ Coim. The constant rank condition translates to $\operatorname{rkT}_{x} f_{\mathrm{NF}}=\mathrm{rkT}_{0} f_{\mathrm{NF}}=\operatorname{dim} \mathrm{Im}$, and thus implies that $\mathrm{T}_{\left(x_{1}, x_{2}\right)} f_{\text {sing }}\left(v_{1}, 0\right)=0$ for all $\left(x_{1}, x_{2}\right) \in U$ and $v_{1} \in$ Ker. Hence, $f_{\text {sing }}\left(x_{1}, x_{2}\right)$ does not depend on $x_{1}$ and so $f_{\text {sing }}\left(x_{1}, x_{2}\right)=f_{\text {sing }}\left(0, x_{2}\right)=0$.

The converse directions are clear.

Using the local structure of submersions and immersions, it is straightforward to verify that the usual statements about the submanifold structure of the naturally induced subsets translate to the locally convex setting. Indeed, according to [31, Theorem C], the level set $f^{-1}(\mu)$ is a submanifold of $M$ if $f$ is a submersion at every $m \in f^{-1}(\mu)$. If instead $f: M \rightarrow N$ is an immersion and a topological embedding, then $f(M)$ is a submanifold of $N$, see [31, Lemma I.13].

The upshot of Proposition 3.3 is that one obtains the submersion, regular value, immersion and constant rank theorem practically for free as soon as one knows that the map under study can be brought into a normal form. A normal form theorem thus unifies these fundamental theorems under one umbrella. In other words, we avoid the renewed construction of special normal forms for each of these results separately, which would be the standard approach in textbooks about (finite-dimensional) differential geometry [47, 51]. The aim of the remainder of the section is to find suitable conditions on the derivative of a map $f$ which ensure that $f$ can be brought into a normal form.

Remark 3.4 (Finite-dimensional reduction and Sard-Smale theorem) As we have seen in (3.2), the singularities of a level set $f^{-1}(\mu)$ are, in terms of a local normal form, completely encoded in the map

$$
f_{\text {sing }}(\cdot, 0): \text { Ker } \rightarrow \text { Coker. }
$$

\footnotetext{
5 An operator $T: X \rightarrow Y$ between locally convex spaces $X$ and $Y$ is said to be of finite-rank if its image is finite-dimensional. In this case, the dimension of $\operatorname{Im} T$ is called the rank of $T$ and it is denoted by $\operatorname{rk} T$.
} 
If $\mathrm{T}_{m} f$ is a Fredholm operator, then Ker and Coker are finite-dimensional spaces. Thus, in this case, the study of the singular structure of $f^{-1}(\mu)$ is reduced to a question in finite dimensions. Exploiting this reduction to finite dimensions, we may mimic the usual proof of the Sard-Smale theorem [76] to obtain a generalization of this theorem to Fredholm maps between locally convex manifolds. We leave the details to the reader.

\subsection{Banach version}

The main idea of the proof of our general normal form theorem is to deform some initially chosen charts in such a way that the singular part of the local representative satisfies the conditions of Definition 3.1. This deformation will be accomplished using the inverse function theorem. To somewhat reduce the functional analytic complexity, we first restrict attention to the Banach setting.

Theorem 3.5 (Normal form-Banach) A smooth map $f: M \rightarrow N$ between Banach manifolds can be brought into a normal form at a point $m \in M$ if and only if $\mathrm{T}_{m} f: \mathrm{T}_{m} M \rightarrow \mathrm{T}_{f(m)} N$ is a regular operator. In particular, every smooth map between finite-dimensional manifolds can be brought into a normal form around every point.

Proof If $f$ can be brought into the normal form $\left(X, Y, \hat{f}, f_{\text {sing }}\right)$, then $\mathrm{T}_{m} f$ coincides up to conjugation by topological isomorphisms with the differential at 0 of $f_{\mathrm{NF}}=\hat{f}+f_{\text {sing }}: X \supseteq U \rightarrow Y$. Now $\mathrm{T}_{0} f_{\mathrm{NF}}=\hat{f} \circ \mathrm{pr}_{\mathrm{Coim}}$ shows that $\mathrm{T}_{m} f$ is regular.

In the other direction, since the claim is of local nature, we can use charts $\tilde{\kappa}: M \supseteq U^{\prime} \rightarrow U \subseteq X$ at $m$ and $\tilde{\rho}: N \supseteq V^{\prime} \rightarrow V \subseteq Y$ at $f(m)$ to replace $f$ by its local representative $\tilde{f}:=\tilde{\rho} \circ f \circ \tilde{\kappa}^{-1}: X \supseteq U \rightarrow Y$, and $\mathrm{T}_{m} f$ by $T:=\mathrm{T}_{0} \tilde{f}: X \rightarrow Y$. Since $T$ is regular by assumption, there exist topological decompositions $X=\operatorname{Ker} T \oplus \operatorname{Coim} T$ and $Y=\operatorname{Coker} T \oplus \operatorname{Im} T$. Moreover, the core $\hat{T}: \operatorname{Coim} T \rightarrow \operatorname{Im} T$ of $T$ is a topological isomorphism.

Define the smooth map $\psi: X \supseteq U \rightarrow X$ by

$$
\psi\left(x_{1}, x_{2}\right)=\left(x_{1}, \hat{T}^{-1} \circ \operatorname{pr}_{\operatorname{Im} T} \circ \tilde{f}\left(x_{1}, x_{2}\right)\right),
$$

with $x_{1} \in \operatorname{Ker} T$ and $x_{2} \in \operatorname{Coim} T$. Note that $\psi(0)=0$. Since $\mathrm{T}_{0} \psi=\mathrm{id}_{X}$, it follows from the Inverse Function Theorem A.1 that we can shrink $U$ in such a way that $\psi(U)$ is an open neighborhood of 0 in $X$ and $\psi: U \rightarrow \psi(U)$ is a diffeomorphism. By possibly shrinking $V$, we may assume that $V \cap \operatorname{Im} T \subseteq \hat{T} \circ \psi(U \cap \operatorname{Coim} T)$. Define the smooth map $\phi: Y \supseteq V \rightarrow Y$ by

$$
\phi\left(y_{1}, y_{2}\right)=\left(y_{1}+\operatorname{pr}_{\operatorname{Coker} T} \circ \tilde{f} \circ \psi^{-1}\left(0, \hat{T}^{-1} y_{2}\right), y_{2}\right)
$$

with $y_{1} \in \operatorname{Coker} T$ and $y_{2} \in \operatorname{Im} T$. A direct calculation shows $\phi(0)=0$ and $\mathrm{T}_{0} \phi=\mathrm{id}_{Y}$. Thus, the Inverse Function Theorem A.1 implies that we can shrink $V$ so that $\phi(V)$ is an open neighborhood of 0 in $Y$ and $\phi: V \rightarrow \phi(V)$ is a diffeomorphism. By possibly shrinking $U$, we may assume $\tilde{f}(U) \subseteq V$ and $\tilde{f}(U) \subseteq \phi(V)$.

Set $\hat{f}:=\hat{T}: \operatorname{Coim} T \rightarrow \operatorname{Im} T$ and define the smooth map $f_{\text {sing }}: X \supseteq \psi(U) \rightarrow \operatorname{Coker} T$ by

$$
f_{\text {sing }} \circ \psi\left(x_{1}, x_{2}\right)=\operatorname{pr}_{\operatorname{Coker} T}\left(\tilde{f}\left(x_{1}, x_{2}\right)-\tilde{f} \circ \psi^{-1}\left(0, \hat{T}^{-1} \circ \operatorname{pr}_{\operatorname{Im} T} \circ \tilde{f}\left(x_{1}, x_{2}\right)\right)\right) .
$$

A straightforward computation shows that the following diagram commutes: 


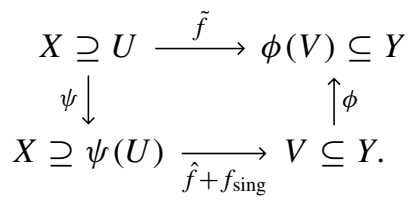

Thus, in the charts $\kappa=\psi \circ \tilde{\kappa}$ and $\rho=\phi^{-1} \circ \tilde{\rho}$, the map $f$ coincides with $f_{\mathrm{NF}}=\hat{f}+f_{\text {sing }}$. This proofs (3.3). Finally, let us verify the asserted properties of the singular part $f_{\text {sing }}$. We clearly have $\mathrm{T}_{0} f_{\text {sing }}=0$. Moreover, for all $x_{2} \in U \cap \operatorname{Coim} T$, we get

$$
\begin{aligned}
f_{\text {sing }} \circ \psi\left(0, x_{2}\right) & =\operatorname{pr}_{\operatorname{Coker} T}\left(\tilde{f}\left(0, x_{2}\right)-\tilde{f} \circ \psi^{-1}\left(0, \hat{T}^{-1} \circ \operatorname{pr}_{\operatorname{Im} T} \circ \tilde{f}\left(0, x_{2}\right)\right)\right) \\
& =\operatorname{pr}_{\operatorname{Coker} T}\left(\tilde{f}\left(0, x_{2}\right)-\tilde{f} \circ \psi^{-1} \circ \psi\left(0, x_{2}\right)\right) \\
& =0 .
\end{aligned}
$$

From $\psi\left(0, x_{2}\right) \in \operatorname{Coim} T$ it follows that $\tilde{f}\left(0, x_{2}^{\prime}\right)=0$ holds for all $x_{2}^{\prime} \in \psi(U) \cap \operatorname{Coim} T$.

The first part of the proof of Theorem 3.5 is inspired by the Lyapunov-Schmidt reduction procedure. To establish the link, let us give a brief outline of this procedure, mostly ignoring the peculiarities of the infinite-dimensional setting, see, e.g., [8, Section 1.3]. Given Banach spaces $X$ and $Y$, and a smooth map $f: X \supseteq U \rightarrow Y$ defined on an open neighborhood $U$ of 0 in $X$ with $f(0)=0$, we are interested in solutions of the nonlinear equation

$$
f(x)=0
$$

near the solution $x=0$. The Lyapunov-Schmidt scheme consists of the following steps:

1. Split $X$ and $Y$ into direct sums $X=\operatorname{Ker} T \oplus \operatorname{Coim} T$ and $Y=\operatorname{Coker} T \oplus \operatorname{Im} T$, where $T=\mathrm{T}_{0} f: X \rightarrow Y$ as above. The equation (3.11) is then equivalent to the system

$$
\begin{gathered}
\operatorname{pr}_{\text {Coker } T} \circ f\left(x_{1}, x_{2}\right)=0, \\
\operatorname{pr}_{\operatorname{Im} T} \circ f\left(x_{1}, x_{2}\right)=0,
\end{gathered}
$$

with $x_{1} \in \operatorname{Ker} T$ and $x_{2} \in \operatorname{Coim} T$.

2. The Implicit Function Theorem shows that, after possibly shrinking $U$, the second equation in (3.12) has a unique solution $x_{2}=x_{2}\left(x_{1}\right) \in U \cap \operatorname{Coim} T$ as a function of $x_{1} \in U \cap \operatorname{Ker} T$.

3. Substituting this solution of the second equation into the first equation of (3.12) yields the reduced equation

$$
\operatorname{pr}_{\text {Coker } T} \circ f\left(x_{1}, x_{2}\left(x_{1}\right)\right)=0 .
$$

for the unknown $x_{1} \in U \cap \operatorname{Ker} T$.

In this way, the nonlinear equation (3.11) is reduced to the nonlinear equation (3.13), which often happens to be a set of finitely many equations for a finite number of unknowns. When comparing this reduction scheme with our construction of the chart deformation $\psi$ in the proof of Theorem 3.5, the only conceptual difference is our usage of the inverse function theorem in place of the Implicit Function Theorem employed in the Lyapunov-Schmidt procedure. Both methods rely fundamentally on the fact that the map $\operatorname{pr}_{\operatorname{Im} T} \circ f: X \supseteq U \rightarrow \operatorname{Im} T$ has a surjective derivative at 0 . In our language, the reduced Eq. (3.13) takes the form 


$$
f_{\text {sing }}\left(x_{1}, 0\right)=0
$$

for $x_{1} \in U \cap \operatorname{Ker} T$.

Similar ideas are also used in the study of deformations of geometric objects, see, e.g., [46] concerning deformations of complex structures and [18], Section II.2; [79], Section 6] in the gauge theoretic setting; see also [19, Section 4.2.5]. In this context, the counterpart of the local diffeomorphism $\psi$ is usually referred to as the Kuranishi map.

\section{Remarks 3.6}

1. A weaker version of Theorem 3.5 can be found in ([49], Theorem 5.1.8; [51], Theorem 2.5.14). There, the chart on $N$ is not modified and hence the additional property $f_{\text {sing }}(0, \cdot)=0$ of the singular part is not deduced. Note that this property was crucial in the proof of Proposition 3.3(2) to show that a smooth map with injective differential is an immersion.

2. By Proposition 3.3(2), we get a corresponding normal form theorem for immersions. Note that in our construction of the normal form the chart on the domain is always deformed, which is in contrast to the classical immersion theorem (e.g., [51, Theorem 2.5.12]). Hence, the constructed submanifold charts differ from the usual ones.

\subsection{Banach target or domain}

In the following, we give generalizations of the Banach normal form theorem to different analytic settings. Let us start with the following extension of Theorem 3.5 to more general domains.

Theorem 3.7 (Normal form-Banach target) Let $f: M \rightarrow N$ be a smooth map, where $M$ is a locally convex manifold and $N$ is a Banach manifold. Then, $f$ can be brought into a normal form at the point $m \in M$ if and only if the differential $\mathrm{T}_{m} f: \mathrm{T}_{m} M \rightarrow \mathrm{T}_{f(m)} N$ is a regular operator. In particular, every smooth map $f: M \rightarrow N$ with finite-dimensional target $N$ can be brought into a normal form at every point.

Proof The proof of Theorem 3.5 carries over word by word except for the part where the inverse function theorem has been used to show that the map $\psi$ defined in (3.6) is a local diffeomorphism. The idea here is to use the Inverse Function Theorem A.2 due to Glöckner [30] instead. For this purpose, define the smooth map $\bar{\psi}: X \supseteq U \rightarrow \operatorname{Coim} T$ by

$$
\bar{\psi}\left(x_{1}, x_{2}\right)=\hat{T}^{-1} \circ \operatorname{pr}_{\operatorname{Im} T} \circ f\left(x_{1}, x_{2}\right) .
$$

The partial derivative of $\bar{\psi}$ at 0 with respect to the second component is given by $\mathrm{T}_{0}^{2} \bar{\psi}=\mathrm{id}_{\mathrm{Coim} T}$. Considering $x_{1} \in$ Ker as a parameter, the Inverse Function Theorem A.2 shows that the map

$$
\psi\left(x_{1}, x_{2}\right)=\left(x_{1}, \bar{\psi}\left(x_{1}, x_{2}\right)\right)
$$

is a local diffeomorphism. Note that $\psi$ coincides with the map defined in (3.6), so that the rest of the proof of Theorem 3.5 goes through without modification. The second part of the 
claim follows from Lemma 2.2, which shows that every linear continuous map with finitedimensional target is a regular operator.

In combination with Proposition 3.3, we recover the submersion theorem and the constant rank theorem [31, Theorem A and F] for maps with values in a finite-dimensional manifold as a special case of Theorem 3.7.

We also have the following version of the normal form theorem where the domain is a Banach manifold.

Theorem 3.8 (Normal form-Banach domain) Let $f: M \rightarrow N$ be a smooth map between manifolds, where $M$ is a Banach manifold and $N$ is a locally convex manifold. Then $f$ can be brought into a normal form at the point $m \in M$ if and only if the differential $\mathrm{T}_{m} f: \mathrm{T}_{m} M \rightarrow \mathrm{T}_{f(m)} N$ is a regular operator. In particular, every smooth map $f: M \rightarrow N$ with $M$ being finite-dimensional can be brought into a normal form around every point.

Proof The proof proceeds similarly to Theorem 3.5 with the modification that Glöckner's Inverse Function Theorem A.2 is used to show that $\phi$ defined in (3.7) is a local diffeomorphism (using a similar strategy as in the proof of Theorem 3.7). Details are left to the reader.

In conjunction with Proposition 3.3(2), we recover the immersion theorem [31, Theorem $\mathrm{H}$ ] for maps from a Banach manifold into a locally convex manifold.

\subsection{Nash-Moser version}

We now establish a normal form theorem in the tame Fréchet category using the Nash-Moser inverse function theorem. For the convenience of the reader, the basic concepts are briefly summarized in Appendix A.

In contrast to the Banach inverse function theorem, the Nash-Moser theorem requires the derivative to be invertible in a whole neighborhood of a given point. This additional condition is due to the fact that the subset of invertible operators is no longer open in the space of all operators. In Sect. 2.1, we have introduced the notion of uniform regularity to address similar problems. Uniform regularity plays a major role in the context of normal forms, too. In fact, the following result has been the main inspiration for this concept.

Proposition 3.9 For every normal form $\left(X, Y, \hat{f}, f_{\text {sing }}\right)$, the family $\mathrm{T}_{x} f_{\mathrm{NF}}: X \rightarrow Y$ of linear maps parametrized by $x \in U \subseteq X$ is uniformly regular at 0 .

Proof With respect to the decompositions $X=\operatorname{Ker} \oplus \operatorname{Coim}$ and $Y=\operatorname{Coker} \oplus \operatorname{Im}$, the derivative of $f_{\mathrm{NF}}$ at $x \in U$ is given in block form as

$$
\mathrm{T}_{x} f_{\mathrm{NF}}=\left(\begin{array}{cc}
\left(\mathrm{T}_{x} f_{\text {sing }}\right)_{\lceil\text {Ker }} & \left(\mathrm{T}_{x} f_{\text {sing }}\right)_{\uparrow \text { Coim }} \\
0 & \hat{f}
\end{array}\right) .
$$


In particular, $\mathrm{T}_{0} f_{\mathrm{NF}}=\hat{f} \circ \mathrm{pr}_{\text {Coim }}$, because $\mathrm{T}_{0} f_{\text {sing }}=0$. This shows that the abstract spaces Coim $\subseteq X$ and Coker $\subseteq Y$ are topological complements of the kernel and image of $\mathrm{T}_{0} f_{\mathrm{NF}}$, respectively. Moreover, the map

$$
\mathrm{pr}_{\mathrm{Im}} \circ\left(\mathrm{T}_{x} f_{\mathrm{NF}}\right)_{\lceil\text {Coim }}: \text { Coim } \rightarrow \operatorname{Im}
$$

coincides with the isomorphism $\hat{f}$, which confirms that $\mathrm{T}_{x} f_{\mathrm{NF}}$ is uniformly regular at 0 .

In order to extend the notion of uniform regularity to the setting of manifolds, consider a morphism $T: E \rightarrow F$ of vector bundles over a manifold $M$. Suppose that $E$ and $F$ are trivialized over an open subset $U \subseteq M$, that is, we are given vector bundle isomorphism $E_{\uparrow U} \rightarrow U \times \underline{E}$ and $F_{\uparrow U} \rightarrow U \times \underline{F}$, where $\underline{E}$ and $\underline{F}$ are locally convex spaces. With respect to these trivializations, we identify $T$ with a family $T_{\uparrow U}: U \times \underline{E} \rightarrow \underline{F}$ of linear maps.

Definition 3.10 A morphism $T: E \rightarrow F$ of vector bundles over $M$ is called uniformly regular at $m \in M$ if there exist local trivializations of $E$ and $F$ on an open neighborhood $U$ of $m$ such that the induced family $T_{\uparrow U}: U \times \underline{E} \rightarrow \underline{F}$ of linear maps is uniformly regular at $m$ in the sense of Definition 2.1. Similarly, a tame morphism $T: E \rightarrow F$ between tame Fréchet vector bundles is called uniformly tame regular at $m$, if there exist tame local trivializations such that the induced family $T_{\mid U}: U \times \underline{E} \rightarrow \underline{F}$ is uniformly tame regular at $m$.

Phrased in this language, Proposition 3.9 entails that the derivative of $f: M \rightarrow N$, viewed as a vector bundle map $\mathrm{T} f: \mathrm{T} M \rightarrow f^{*} \mathrm{~T} N$ over $M$, is uniformly regular at $m \in M$ if $f$ can be brought into a normal form at $m$. The following theorem shows that, in the tame category, uniform regularity of the derivative is also a sufficient condition for the existence of a normal form.

Theorem 3.11 (Normal form-Tame Fréchet) A tame smooth map $f: M \rightarrow N$ between tame Fréchet manifolds can be brought into a tame normal form at the point $m \in M$ if and only if $\mathrm{T} f: \mathrm{T} M \rightarrow f^{*} \mathrm{~T} N$ is uniformly tame regular at $m$.

Proof If $f$ can be brought into a tame normal form, then Proposition 3.9 (phrased in the tame category) shows that $\mathrm{T} f: \mathrm{T} M \rightarrow f^{*} \mathrm{~T} N$ is uniformly tame regular at $m$.

The proof of the converse direction follows the same line of arguments as the proof of Theorem 3.5 except that we will use the Nash-Moser theorem A.3 to show that the chart deformations (3.6) and (3.7) are local diffeomorphisms. Continuing in the notation of the proof of Theorem 3.5, abbreviate $T_{x} \equiv \mathrm{T}_{x} f: X \rightarrow Y$ for every $x \in U \subseteq X$. The assumption of uniform tame regularity of $\mathrm{T} f$ implies that the family $T: U \times X \rightarrow Y$ is uniformly tame regular at 0 .

The derivative at $x \in U$ of the map $\psi$ defined in (3.6) evaluates to

$$
\mathrm{T}_{x} \psi=\left(\begin{array}{cc}
\operatorname{id}_{\operatorname{Ker} T_{0}} & 0 \\
\hat{T}_{0}^{-1} \circ \operatorname{pr}_{\operatorname{Im} T_{0}} \circ\left(T_{x}\right)_{\mid \operatorname{Ker} T_{0}} & \hat{T}_{0}^{-1} \circ \tilde{T}_{x}
\end{array}\right),
$$

where $\tilde{T}_{x}=\operatorname{pr}_{\operatorname{Im} T_{0}} \circ\left(T_{x}\right)_{\left\lceil\operatorname{Coim} T_{0}\right.}: \operatorname{Coim} T_{0} \rightarrow \operatorname{Im} T_{0}$. Since $T$ is uniformly tame regular, $\tilde{T}_{x}$ is invertible for all $x \in U$ and the inverses form a tame smooth family. Hence, $\mathrm{T}_{x} \psi$ has a tame smooth family of inverses given by 


$$
\left(\mathrm{T}_{x} \psi\right)^{-1}=\left(\begin{array}{cc}
\operatorname{id}_{\operatorname{Ker} T_{0}} & 0 \\
-\tilde{T}_{x}^{-1} \circ \operatorname{pr}_{\operatorname{Im} T_{0}} \circ\left(T_{x}\right)_{\left\lceil\operatorname{Ker} T_{0}\right.} & \tilde{T}_{x}^{-1} \circ \hat{T}_{0}
\end{array}\right) .
$$

Thus, we can apply the Nash-Moser Theorem A.3 to conclude that $\psi$ is a local diffeomorphism at 0 .

Using (3.20), the derivative of the map $\phi$ defined in (3.7) can be written in block form as

$$
\mathrm{T}_{y} \phi=\left(\begin{array}{cc}
\operatorname{id}_{\operatorname{Coker} T_{0}} & \operatorname{pr}_{\operatorname{Coker} T_{0}} \circ T_{x} \circ \tilde{T}_{x}^{-1} \\
0 & \operatorname{id}_{\operatorname{Im} T_{0}}
\end{array}\right),
$$

where $y \in V \subseteq Y$ and $x=\psi^{-1}\left(0, \hat{T}^{-1} \circ \operatorname{pr}_{\operatorname{Im} T_{0}}(y)\right)$. A direct calculation verifies that $\mathrm{T}_{y} \phi$ is invertible with inverse given by

$$
\left(\mathrm{T}_{y} \phi\right)^{-1}=\left(\begin{array}{cc}
\operatorname{id}_{\operatorname{Coker} T_{0}} & -\operatorname{pr}_{\operatorname{Coker} T_{0}} \circ T_{x} \circ \tilde{T}_{x}^{-1} \\
0 & \operatorname{id}_{\operatorname{Im} T_{0}}
\end{array}\right) .
$$

Since the inverses $\left(\mathrm{T}_{y} \phi\right)^{-1}$ parametrized by $y \in V$ form a tame smooth family, the NashMoser Theorem A.3 implies that $\phi$ is a local diffeomorphism. The remainder of the proof of Theorem 3.5 goes through without modification.

\subsection{Elliptic version}

In applications to geometry and physics, one is usually interested in differential operators between spaces of geometric objects. Let $E \rightarrow M$ and $F \rightarrow M$ be finite-dimensional fiber bundles over the compact manifold $M$. Denote the space of smooth sections of $E$ and $F$ by $\mathcal{E}$ and $\mathcal{F}$, respectively. By [35, Theorem II.2.3.1], the spaces $\mathcal{E}$ and $\mathcal{F}$ are tame Fréchet manifolds. Following [63, Definition 15.3], a nonlinear differential operator of degree $r$ is a map $\mathcal{E} \rightarrow \mathcal{F}$ factorizing through the $r$-jet bundle $\mathrm{J}^{r} E$. That is, for every vertical morphism $f: \mathrm{J}^{r} E \rightarrow F$ of fiber bundles the associated nonlinear differential operator is the composition

$$
L: \mathcal{E} \stackrel{\mathrm{j}^{r}}{\longrightarrow} \Gamma^{\infty}\left(\mathrm{J}^{r} E\right) \stackrel{f_{*}}{\longrightarrow} \mathcal{F},
$$

where $\mathrm{j}^{r}$ denotes the $r$-th jet prolongation. This is similar to the factorization (2.26) in the linear case, with the important difference that $f$ is now a nonlinear fiber bundle morphism. Every nonlinear differential operator $L_{f}: \mathcal{E} \rightarrow \mathcal{F}$ is a tame smooth map according to [35, Corollary II.2.2.7].

As one would expect, the linearization of a nonlinear differential operator is a linear differential operator. In fact, the differential $\mathrm{T}_{\phi} L_{f}$ is given by

$$
\begin{aligned}
\mathrm{T}_{\phi} L_{f}: \Gamma^{\infty}\left(\phi^{*} \mathrm{~V} E\right) \stackrel{\mathrm{j}^{r}}{\longrightarrow} & \Gamma^{\infty}\left(\mathrm{J}^{r}\left(\phi^{*} \mathrm{~V} E\right)\right) \\
\downarrow & \underline{ } \\
& \Gamma^{\infty}\left(\left(\mathrm{j}^{r} \phi\right)^{*} \mathrm{~V}\left(\mathrm{~J}^{r} E\right)\right) \stackrel{(\mathrm{V} f)_{*}^{*}}{\longrightarrow} \Gamma^{\infty}\left(\left(L_{f}(\phi)\right)^{*} \mathrm{~V} F\right),
\end{aligned}
$$

where $\mathrm{V} E$ denotes the vertical subbundle of $\mathrm{T} E$ and $\mathrm{V} f: \mathrm{V}\left(\mathrm{J}^{r} E\right) \rightarrow f^{*} \mathrm{~V} F$ is the vertical derivative of $f$. The isomorphism at the center is induced by the natural isomorphism of 
$\left(\mathrm{j}^{r} \phi\right)^{*} \mathrm{~V}\left(\mathrm{~J}^{r} E\right)$ and $\mathrm{J}^{r}\left(\phi^{*} \mathrm{~V} E\right)$, see [63, Theorem 17.1]. Hence, $\mathrm{T}_{\phi} L_{f}$ is a linear differential operator with coefficients $\mathrm{V} f$.

We say that $L_{f}$ is elliptic if the linear differential operator $\mathrm{T}_{\phi} L_{f}$ is elliptic for all $\phi \in \mathcal{E}$.

Theorem 3.12 (Normal form-Elliptic) Let $E \rightarrow M$ and $F \rightarrow M$ be finite-dimensional fiber bundles over the compact manifold $M$. Every nonlinear elliptic differential operator $L_{f}: \mathcal{E} \rightarrow \mathcal{F}$ can be brought into a tame normal form at every $\phi \in \mathcal{E}$.

Proof According to Theorem 3.11, we have to show that the bundle map

$$
\mathrm{T} L_{f}: \mathrm{T} \mathcal{E} \rightarrow\left(L_{f}\right)^{*} \mathrm{~T} \mathcal{F}
$$

is uniformly tame regular at $\phi \in \mathcal{E}$. After having chosen tubular neighborhoods of $\operatorname{Im} \phi \subseteq E$ and $\operatorname{Im} L_{f}(\phi) \subseteq F$, it suffices to consider the case where $E$ and $F$ are vector bundles. In this linear setting, the vertical tangent bundle of $\mathrm{J}^{r} E$ is identified with $\mathrm{V}\left(\mathrm{J}^{r} E\right) \simeq \mathrm{J}^{r} E \times{ }_{M} \mathrm{~J}^{r} E$. Accordingly, the vertical derivative of $f$ is a map $\mathrm{V} f: \mathrm{J}^{r} E \times_{M} \mathrm{~J}^{r} E \rightarrow F$. Define the tame smooth family $T: \mathcal{E} \times \mathcal{E} \rightarrow \mathcal{F}$ of linear maps by

$$
T(\varphi, \sigma) \equiv T_{\varphi}(\sigma)=\mathrm{V} f\left(\mathrm{j}^{r} \varphi, \mathrm{j}^{r} \sigma\right) .
$$

We have to show that the family $\varphi \mapsto T_{\varphi}$ is uniformly tame regular at $\varphi=\phi$. For this purpose, note that $T$ factorizes as the composition of the tame smooth map

$$
\mathcal{E} \times \mathcal{E} \ni(\varphi, \sigma) \mapsto\left((\mathrm{V} f)_{*}\left(\mathrm{j}^{r} \varphi, \cdot\right), \sigma\right) \in \Gamma^{\infty}\left(\mathrm{L}\left(\mathrm{J}^{r} E, F\right)\right) \times \mathcal{E}
$$

and the family of differential operators

$$
D: \Gamma^{\infty}\left(\mathrm{L}\left(\mathrm{J}^{r} E, F\right)\right) \times \mathcal{E} \rightarrow \mathcal{F}, \quad(\Lambda, \sigma) \mapsto \Lambda\left(\mathrm{j}^{r} \sigma\right) .
$$

By assumption, the differential operator with coefficients $\Lambda_{\phi}=(\mathrm{V} f)_{*}\left(\mathrm{j}^{r} \phi, \cdot\right)$ is elliptic. Thus, Theorem 2.12 implies that $D$ is uniformly tame regular at $\Lambda_{\phi}$, and hence that the family $T$ is uniformly tame regular at $\varphi=\phi$.

In applications, one often encounters infinite-dimensional manifolds which are not quite spaces of sections of a fiber bundle. A prime example is the Fréchet Lie group of diffeomorphisms of a compact manifold. Note that the diffeomorphism group is locally modeled on the space of vector fields. Thus, from the perspective of local normal forms, it has a very similar structure to the space of sections. In order to extend the normal form theorem to such situations, we follow [77, p. 57] and introduce the following subclass of Fréchet manifolds.

Definition 3.13 A tame Fréchet manifold $M$ is said to be geometric if it is locally modeled on the space of smooth sections of some vector bundle over a compact manifold.

A tame smooth map $f: M \rightarrow N$ is called geometric if for every point $m \in M$ there exist vector bundles $E$ and $F$ over the same compact manifold, and tame local trivializations $(\mathrm{T} M)_{\uparrow U} \simeq U \times \Gamma^{\infty}(E)$ and $\left(f^{*} \mathrm{~T} N\right)_{\uparrow U} \simeq U \times \Gamma^{\infty}(F)$ in a neighborhood $U \subseteq M$ of $m$ such that in this trivialization the derivative $\mathrm{T} f: \mathrm{T} M \rightarrow f^{*} \mathrm{~T} N$ factorizes as the composition of a tame smooth map $U \rightarrow \Gamma^{\infty}\left(\mathrm{L}\left(\mathrm{J}^{r} E, F\right)\right)$ and the family of differential operators 


$$
D: \Gamma^{\infty}\left(\mathrm{L}\left(\mathrm{J}^{r} E, F\right)\right) \times \Gamma^{\infty}(E) \rightarrow \Gamma^{\infty}(F), \quad(\Lambda, \sigma) \mapsto \Lambda\left(\mathrm{j}^{r} \sigma\right) .
$$

The notions of a geometric vector bundle and a geometric vector bundle morphism are defined in a similar way.

Roughly speaking, a map $f: M \rightarrow N$ is geometric if its linearization $\mathrm{T}_{m} f$ is a family of linear differential operators whose coefficients depend tamely on $m \in M$. As we have seen in the proof of Theorem 3.12, every nonlinear differential operator is a geometric map. Moreover, a slight reformulation of the proof of Theorem 3.12 gives the following more general normal form result.

Theorem 3.14 (Normal form-Elliptic) Let $f: M \rightarrow N$ be a geometric map between geometric Fréchet manifolds. If $\mathrm{T}_{m} f: \mathrm{T}_{m} M \rightarrow \mathrm{T}_{f(m)} N$ is an elliptic differential operator for some $m \in M$, then $f$ can be brought into a tame normal form at $m$.

Proof By Theorem 3.11, we have to show that the bundle map $\mathrm{T} f: \mathrm{T} M \rightarrow f^{*} \mathrm{~T} N$ is uniformly tame regular at $m$. Since $f$ is geometric, there exist tame local trivializations $(\mathrm{T} M)_{\uparrow U} \simeq U \times \Gamma^{\infty}(E)$ and $\left(f^{*} \mathrm{~T} N\right)_{\uparrow U} \simeq U \times \Gamma^{\infty}(F)$ in a neighborhood $U \subseteq M$ of $m$ such that $\mathrm{T} f$ factorizes as the composition of a tame smooth map $S: U \rightarrow \Gamma^{\infty}\left(\mathrm{L}\left(\mathrm{J}^{r} E, F\right)\right)$ and the family of differential operators $D: \Gamma^{\infty}\left(\mathrm{L}\left(\mathrm{J}^{r} E, F\right)\right) \times \Gamma^{\infty}(E) \rightarrow \Gamma^{\infty}(F)$ defined in (3.29). By assumption, the differential operator $D_{S(m)}$ with symbol $S(m)$ is elliptic. Hence, Theorem 2.12 implies that the family $\mathrm{T} f$ is uniformly tame regular at $m$.

\subsection{Relative normal form}

We now briefly discuss how to bring a smooth map $f: M \rightarrow N$ into a normal form relative to a given submanifold $P$ of $N$. This is a direct generalization of the standard transversality theory. Recall that a submanifold $P \subseteq N$ is called split if $\mathrm{T}_{p} P$ is topologically complemented in $\mathrm{T}_{p} N$ for every point $p \in P$. Let $\mathrm{pr}_{p}: \mathrm{T}_{p} N \rightarrow \mathrm{T}_{p} N / \mathrm{T}_{p} P$ denote the canonical projection. For the special case where $P$ is a single point, the following relative normal form result recovers Theorem 3.5.

Theorem 3.15 (Relative normal form - Banach) Let $f: M \rightarrow N$ be a smooth map between Banach manifolds, and let $P$ be a split submanifold of $N$. If the operator $\operatorname{pr}_{f(m)} \circ \mathrm{T}_{m} f: \mathrm{T}_{m} M \rightarrow \mathrm{T}_{f(m)} N / \mathrm{T}_{f(m)} P$ is regular at the point $m \in M$, then there exist charts $\kappa: M \supseteq U^{\prime} \rightarrow U \subseteq X$ at $m$ and $\rho: N \supseteq V^{\prime} \rightarrow V \subseteq Y$ at $f(m)$, and decompositions $X=\operatorname{Ker} \oplus \operatorname{Coim}, Y=$ Coker $\oplus \operatorname{Im} \oplus Z$ such that

$$
\rho \circ f \circ \kappa^{-1}=\hat{f}+f_{\text {sing }}+f_{P},
$$

where $\hat{f}:$ Coim $\rightarrow \operatorname{Im}$ is a linear topological isomorphism, $f_{\text {sing }}: X \supseteq U \rightarrow$ Coker is a smooth map satisfying $f_{\text {sing }}\left(0, x_{2}\right)=0$ for all $x_{2} \in U \cap$ Coim and $\mathrm{T}_{0} f_{\text {sing }}=0$, and $f_{P}: X \supseteq U \rightarrow Z$ is a smooth map. The chart $\rho$ can be chosen to be a submanifold chart for $P$, i.e., $\rho\left(P \cap V^{\prime}\right)=Z \cap V$. 

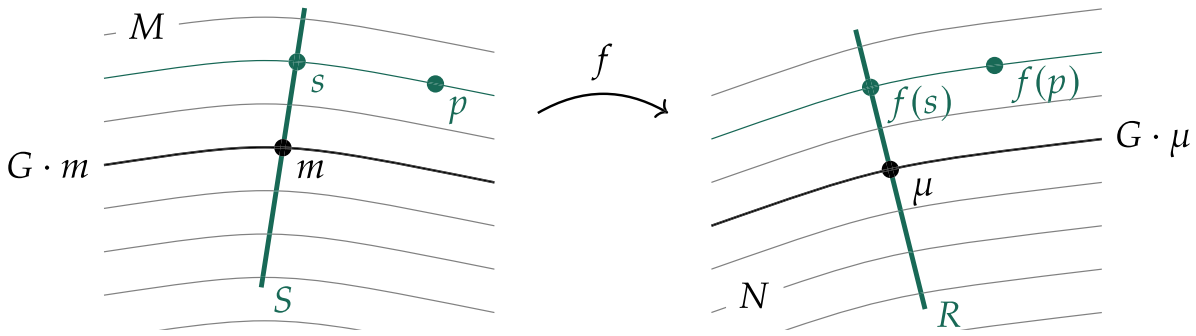

Fig. 1 Illustration of the idea to capture the part of $f$ that does not come from the group action in a map $f_{S}^{R}: S \rightarrow R$ between the slices $S$ and $R$

Proof Since $P$ is a split submanifold of $N$, there exist a chart $\tilde{\rho}: N \supseteq V^{\prime} \rightarrow V \subseteq Y$ at $f(m)$, and a topological decomposition $Y=\bar{Y} \oplus Z$ such that $\tilde{\rho}\left(P \cap V^{\prime}\right)=Z \cap V$. Define $\bar{f}:=\operatorname{pr}_{\bar{Y}} \circ \tilde{\rho} \circ f_{\uparrow U^{\prime}}: M \supseteq U^{\prime} \rightarrow \bar{Y}$ and $f_{P}:=\operatorname{pr}_{Z} \circ \tilde{\rho} \circ f_{\uparrow U^{\prime}}: M \supseteq U^{\prime} \rightarrow Z$ where $U^{\prime}$ is a sufficiently small neighborhood of $m$ so that $f\left(U^{\prime}\right) \subseteq V^{\prime}$. Since $\operatorname{pr}_{f(m)} \circ \mathrm{T}_{m} f$ coincides with $\mathrm{T}_{m} \bar{f}$ under the isomorphism $\mathrm{T}_{f(m)} N / \mathrm{T}_{f(m)} P \simeq \bar{Y}$, the operator $\mathrm{T}_{m} \vec{f}$ is regular. The claim now follows from Theorem 3.5 applied to $\bar{f}$.

Since $\rho$ is a submanifold chart for $P$ and $f_{P}$ takes values in $Z$, the identity (3.30) implies

$$
\kappa\left(f^{-1}(P)\right)=\left\{\left(x_{1}, 0\right) \in U: f_{\text {sing }}\left(x_{1}, 0\right)=0\right\} .
$$

Note that $\mathrm{Ker} \simeq\left(\mathrm{T}_{m} f\right)^{-1}\left(\mathrm{~T}_{f(m)} P\right)$. Moreover, Coker is isomorphic to the quotient of $\mathrm{T}_{f(m)} N$ by $\operatorname{ImT}_{m} f+\mathrm{T}_{f(m)} P$. We hence recover the classical result that $f^{-1}(P)$ is a submanifold of $M$ if $f$ is transversal to $P$.

The strategy in the proof of Theorem 3.15 generalizes to the other analytic settings considered above, leading to relative versions of Theorems 3.7, 3.8, 3.11, 3.12 and 3.14. We leave the details to the reader.

\section{Normal form of an equivariant map}

In this section, we study the local properties of a smooth equivariant map. We refer the reader to Appendix B for relevant background material on Lie group actions in infinite dimensions. Consider the following setup. Let $G$ be a Lie group, and let $M$ and $N$ be $G$-manifolds. Assume that the $G$-action on $M$ is proper. Let $f: M \rightarrow N$ be a smooth equivariant map. Choose a point $m \in M$ and denote its image under $f$ by $\mu=f(m) \in N$.

Let us start by describing the general idea of how to construct an equivariant normal form of $f$. Since a slice provides a normal form for the $G$-action in a neighborhood of a given orbit, it naturally comes to mind as a tool for studying equivariant maps. An initial idea would be to split-off the $G$-action with the help of slices $S$ and $R$ for the $G$-action at the points $m \in M$ and $\mu \in N$, respectively. If the slices satisfy $f(S) \subseteq R$, then the part of $f$ that does not come from the group action is captured in the map $f_{S}^{R}=f_{\uparrow S}: S \rightarrow R$ between the slices (see Fig. 1). For the reduced map $f_{S}^{R}$, we can use the normal form results in Sect. 3 to arrive at an equivariant normal form for $f$. In the finite-dimensional setting, a similar strategy has been used in [65, Proposition 2.6] to establish an equivariant submersion theorem under the assumption that $G$ is compact. 
However, for the applications we are interested in, it is often too restrictive to assume that the $G$-action on the target has a slice $R$. For example, momentum maps in symplectic geometry take values in the dual of the Lie algebra; but the coadjoint action of a noncompact group usually does not possess slices, see, e.g., [33, Point 5 in Section 2.3.1]. As a solution, one may try to use only a slice $S$ for the $G$-action on $M$ while leaving the action on $N$ untouched. In the context of symplectic reduction, this idea is at the heart of the proof of the Marle-Guillemin-Sternberg normal form of the momentum map. In this case, the slice $S$ is constructed using an equivariant version of Darboux's theorem. However, a close inspection of the proof of the singular symplectic reduction theorem reveals that the Marle-Guillemin-Sternberg Theorem gives actually not the most convenient normal form for the study of the geometry of the symplectic quotient. In fact, several issues arise from using a slice for the $G$-action but taking the quotient with respect to the subgroup $G_{\mu}$. This asymmetry was counterbalanced in the proof of the reduction theorem [61, Proposition 8.1.2] by deforming the momentum map $J: M \rightarrow \mathfrak{g}^{*}$ using a local diffeomorphism of $\mathfrak{g}^{*}$. Instead of changing coordinates on the target, we will take a different approach and use a slice on $M$ for the action of $G_{\mu}$ instead of $G$. This has also the advantage that $G_{\mu}$ is often considerably smaller than $G$ and, thus, a slice for the subgroup action is easier to construct.

Finally, the symplectic setting is special in that the action of $G$ on $\mathfrak{g}^{*}$ is linear. When moving beyond the momentum map example, the $G$-action on the target $N$ is usually nonlinear. As we have explained above, we cannot use a slice to control the entire $G$-action on $N$. But the stabilizer $G_{m}$ of $m$ is compact and its action on $N$ leaves $\mu$ invariant due to $G$-equivariance of $f$. Hence, we can at least hope to linearize the induced action of $G_{m}$ around the fixed point $\mu$.

In summary, we will work with a $G_{\mu}$-slice $S$ at $m \in M$ and with a $G_{m}$-slice at $\mu \in N$ and then bring the restriction of $f$ to the slice $S$ in a normal form using the results in Sect. 3 .

\subsection{General normal form theorem}

Using this strategy, we will show that a wide class of equivariant maps can be brought into the following equivariant normal form.

Definition 4.1 An abstract equivariant normal form is a tuple $\left(H, X, Y, \hat{f}, f_{\text {sing }}\right)$ consisting of a compact Lie group $H$ and a normal form $\left(X, Y, \hat{f}, f_{\text {sing }}\right)$ which is $H$-equivariant in the sense that $X, Y$ are endowed with smooth linear $H$-actions, the decompositions $X=\operatorname{Ker} \oplus$ Coim, $Y=$ Coker $\oplus \operatorname{Im}$ are $H$-invariant, and the maps $\hat{f}$ : Coim $\rightarrow \operatorname{Im}$, $f_{\text {sing }}: X \supseteq U \rightarrow$ Coker are $H$-equivariant, where $U \subseteq X$ is an $H$-invariant open neighborhood of 0 .

For an equivariant normal form $\left(H, X, Y, \hat{f}, f_{\text {sing }}\right)$ and a Lie group $G$ with $H \subseteq G$, define the smooth map $f_{G \text {-NF }}: G \times_{H} U \rightarrow G \times_{H} Y$ by

$$
f_{G-\mathrm{NF}}\left(\left[g, x_{1}, x_{2}\right]\right):=\left[g, f_{\mathrm{NF}}\left(x_{1}, x_{2}\right)\right]=\left[g, \hat{f}\left(x_{2}\right)+f_{\text {sing }}\left(x_{1}, x_{2}\right)\right]
$$

for $g \in G, x_{1} \in U \cap \operatorname{Ker}$ and $x_{2} \in U \cap$ Coim.

A slice for the $G_{\mu}$-action on $M$ plays a fundamental role in connecting an equivariant map with its equivariant normal form. Recall from Appendix B, that a $G_{\mu}$-slice at $m \in M$ is a $G_{m}$-invariant submanifold $S$ of $M$ which is transverse to the orbit $G_{\mu} \cdot m$ and which possesses some additional properties, see Definition B.1. A proper action of a 
finite-dimensional Lie group on a finite-dimensional manifold always admit slices [62], but this may no longer be true in infinite dimensions and additional assumptions have to be made, see [16]. In the following, we assume the existence of a slice for the $G_{\mu}$-action at every point $m \in M$. In particular, the natural fibration $G_{\mu} \rightarrow G_{\mu} / G_{m}$ is a locally trivial principal bundle and it admits a local section $\chi: G_{\mu} / G_{m} \supseteq W \rightarrow G_{\mu}$ defined on an open neighborhood $W$ of the identity coset $[e]$ in such a way that the map

$$
\chi^{S}: W \times S \rightarrow M, \quad([g], s) \mapsto \chi([g]) \cdot s
$$

is a diffeomorphism onto an open neighborhood of $m$, i.e., the slice yields convenient product coordinates in a neighborhood of $m$. This product structure extends to a semi-local normal form of the orbit using the tube map

$$
\chi^{\mathrm{T}}: G_{\mu} \times_{G_{m}} S \rightarrow M, \quad[g, s] \mapsto g \cdot s,
$$

which is a $G_{\mu}$-equivariant diffeomorphism onto an open, $G_{\mu}$-invariant neighborhood of $G_{\mu} \cdot m$ in $M$, see Proposition B.2.

Definition 4.2 Let $f: M \rightarrow N$ be a smooth $G$-equivariant map. For $m \in M$ write $\mu=f(m) \in N$, and assume that the stabilizer $G_{\mu}$ is a Lie subgroup of $G$. We say that $f$ can be brought into the equivariant normal form $\left(H, X, Y, \hat{f}, f_{\text {sing }}\right)$ at $m$ if $H=G_{m}$ and there exist

1. a slice $S$ at $m$ for the $G_{\mu}$-action,

2. a $G_{m}$-equivariant diffeomorphism $l_{S}: X \supseteq U \rightarrow S \subseteq M$,

3. a $G_{m}$-equivariant chart $\rho: N \supseteq V^{\prime} \rightarrow V \subseteq Y$ at $\mu$ with $f(S) \subseteq V^{\prime}$

such that the following diagram commutes:

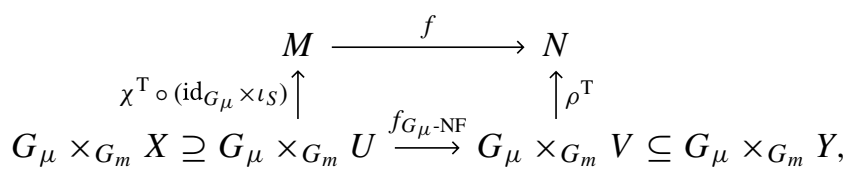

where $\chi^{\mathrm{T}}: G_{\mu} \times_{G_{m}} S \rightarrow M$ is the tube diffeomorphism associated with $S$ and $\rho^{\mathrm{T}}: G_{\mu} \times_{G_{m}} V \rightarrow N$ is defined by $\rho^{\mathrm{T}}([g, y])=g \cdot \rho^{-1}(y)$. For short, we say that $f$ is locally $G$-equivalent to $f_{G_{\mu} \text {-NF }}$.

Assume that the $G$-equivariant map $f: M \rightarrow N$ can be brought into the equivariant normal form $\left(G_{m}, X, Y, \hat{f}, f_{\text {sing }}\right)$ by using diffeomorphisms $l_{S}$ and $\rho$ as above. Then $\mathrm{T}_{m} l_{S}: X \rightarrow \mathrm{T}_{m} S$ identifies $X$ with $\mathrm{T}_{m} S \simeq \mathrm{T}_{m} M / \mathfrak{g}_{\mu} \cdot m$, as $G_{m}$-representations. Similarly, $\mathrm{T}_{\mu} \rho: \mathrm{T}_{\mu} N \rightarrow Y$ is an isomorphism of $G_{m}$-representations. Under these identifications, the abstract spaces Ker and Coker occurring in the decomposition of $X$ and $Y$ are identified with

$$
\operatorname{Ker} \simeq \operatorname{Ker} \mathrm{T}_{m} f / \mathfrak{g}_{\mu} \cdot m, \quad \text { Coker } \simeq \mathrm{T}_{\mu} N / \operatorname{Im~T}_{m} f .
$$

These are the first and second homology groups of the chain complex

$$
0 \longrightarrow \mathfrak{g}_{\mu} \stackrel{\mathrm{T}_{e} \Upsilon_{m}}{\longrightarrow} \mathrm{T}_{m} M \stackrel{\mathrm{T}_{m} f}{\longrightarrow} \mathrm{T}_{\mu} N \longrightarrow 0,
$$


where $\Upsilon_{m}: G_{\mu} \rightarrow M$ is the orbit map of the $G_{\mu}$-action at $m \in M$.

At a fixed point for the action, an equivariant normal form signifies that there exist charts which linearize the actions and at the same time bring $f$ into a normal form (in the sense of Definition 3.2). In fact, the general case can always be reduced to constructing an equivariant normal form at a fixed point of the action.

Proposition 4.3 Let $f: M \rightarrow N$ be an equivariant map between G-manifolds. Choose $m \in M$ and set $\mu=f(m)$. Assume that the following conditions hold:

1. The stabilizer subgroup $G_{\mu}$ of $\mu$ is a Lie subgroup of $G$.

2. The induced $G_{\mu}$-action on $M$ admits a slice $S$ at $m$.

3. The $G_{m}$-equivariant map $f^{S} \equiv f_{\uparrow S}: S \rightarrow N$ can be brought into an equivariant normal form at $m$ relative to the induced $G_{m}$-action, i.e., it is locally $G_{m}$-equivalent to a map $f_{G_{m} \mathrm{NF}}: X \supseteq U \rightarrow Y$.

Then, $f$ can be brought into an equivariant normal form at $m$ relative to the $G$-action. In fact, $f$ is locally $G$-equivalent to

$$
f_{G_{\mu}-\mathrm{NF}}: G_{\mu} \times_{G_{m}} U \rightarrow G_{\mu} \times_{G_{m}} Y, \quad[g, x] \mapsto\left[g, f_{G_{m}-\mathrm{NF}}^{S}(x)\right] .
$$

Proof Let $S$ be a slice at $m$ for the induced $G_{\mu}$-action on $M$, with associated tube map $\chi^{\mathrm{T}}: G_{\mu} \times_{G_{m}} S \rightarrow M$ defined by $\chi^{\mathrm{T}}([g, s])=g \cdot s$. By $G$-equivariance of $f$, the following diagram commutes:

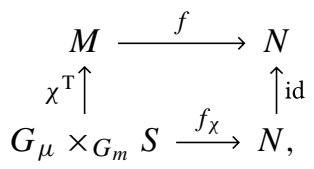

where the smooth map $f_{\chi}$ is defined by $f_{\chi}([g, s])=g \cdot f(s)$ for $g \in G_{\mu}$ and $s \in S$. Thus, the map $f$ decomposes into the $G_{\mu}$-action and the restriction $f^{S}: S \rightarrow N$ of $f$ to the slice. Note that $f^{S}$ is $G_{m}$-equivariant. By assumption, $f^{S}$ can be brought into a $G_{m}$-equivariant normal form. Since $m$ is a fixed point for the $G_{m}$-action, this means that there exists charts $\kappa: S \supseteq U^{\prime} \rightarrow U \subseteq X$ at $m$ and $\rho: N \supseteq V^{\prime} \rightarrow V \subseteq Y$ at $\mu$ intertwining the $G_{m}$-actions on $S$ and $N$ with a linear $G_{m}$-actions on $X$ and $Y$, respectively. Moreover, $f\left(U^{\prime}\right) \subseteq V^{\prime}$ and the following diagram commutes

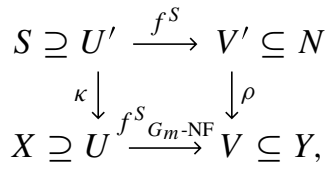

where $f_{G_{m} \text {-NF }}^{S}=\hat{f}_{S}+f^{S}{ }_{\text {sing }}$ with maps $\hat{f}^{S}$ and $f^{S}$ sing as in Definition 4.1. By possibly shrinking $S$, we may assume that $\kappa$ is defined on the whole of $S$. Define $\rho^{\mathrm{T}}: G_{\mu} \times_{G_{m}} V \rightarrow N$ by $\rho^{\mathrm{T}}([g, y])=g \cdot \rho^{-1}(y)$. Due to $G_{m}$-equivariance of $f^{S}, \kappa$ and $\rho$, the prescription 


$$
f_{G_{\mu}-\mathrm{NF}}([g, x])=\left[g, \rho \circ f^{S} \circ \kappa^{-1}(x)\right], \quad g \in G_{\mu}, x \in U
$$

defines a smooth map $f_{G_{\mu}-\mathrm{NF}}: G_{\mu} \times_{G_{m}} U \rightarrow G_{\mu} \times_{G_{m}} V$ fitting into the following commutative diagram:

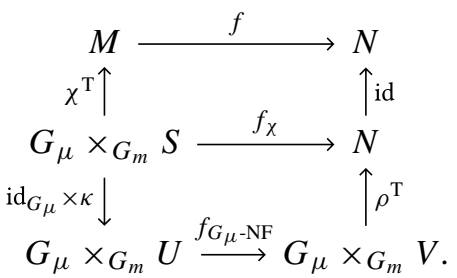

Hence $f$ is brought into the equivariant normal form $\left(G_{m}, X, Y, \hat{f}^{S}, f^{S}{ }_{\text {sing }}\right)$.

At this point, we have completely split off the $G_{\mu}$-action and reduced the problem to constructing an equivariant normal form of the map $f^{S}: S \rightarrow N$ at a fixed point of the Lie group $G_{m}$. This is a considerable simplification of the general situation we started with. In the proposition below, we show that equivariant normal forms at fixed points can be constructed using the methods developed in Sect. 3. For this we will need the following notion.

Definition 4.4 We say that a $G$-action can be linearized at a fixed point $m \in M$ if there exist a $G$-invariant open neighborhood $U^{\prime}$ of $m$ in $M$, a chart $\rho: M \supseteq U^{\prime} \rightarrow U \subseteq X$ and a smooth linear $G$-action on $X$ such that $\rho$ is $G$-equivariant.

Proposition 4.5 Let $G$ be compact Lie group, and let $f: M \rightarrow N$ be an equivariant map between Fréchet $G$-manifolds. Choose $m \in M$ and set $\mu=f(m) \in N$. Assume that $m$ is a fixed point of the $G$-action, i.e., $G_{m}=G$, and that the following conditions hold:

1. The G-actions on $M$ and $N$ can be linearized at the fixed points $m$ and $\mu$, respectively.

2. The hypotheses of either Theorems 3.5, 3.7, 3.8, 3.11, 3.12 and 3.14 are satisfied so that f can be brought into a normal form (not necessarily equivariant).

Then $f$ can be brought into an equivariant normal form at $m$.

Proof By assumption, there exist charts $\tilde{\kappa}: M \supseteq U^{\prime} \rightarrow U \subseteq X$ at $m$ and $\tilde{\rho}: N \supseteq V^{\prime} \rightarrow V \subseteq Y$ at $\mu$ which are $G$-equivariant with respect to linear actions of $G$ on $X$ and $Y$, respectively. All the mentioned normal forms established in Sect. 3 use the chart deformations $\psi$ and $\phi$ introduced in the proof of Theorem 3.5 to bring $f$ into an normal form. We claim that in the present setting these local diffeomorphisms can be chosen to be $G$-equivariant. To see this, consider the topological decompositions $X=\operatorname{Ker} T \oplus \operatorname{Coim} T$ and $Y=\operatorname{Coker} T \oplus \operatorname{Im} T$, where $T$ corresponds to $\mathrm{T}_{m} f$ under the chart identifications. Since $G$ is compact and $T$ is $G$-equivariant, the complements $\operatorname{Coim} T$ and $\operatorname{Coker} T$ can be chosen to be $G$-invariant according to [16, Lemma 3.13]. By direct inspection, we see that the map $\psi$ defined in (3.6) is a composition of $G$-equivariant maps and hence is $G$-equivariant. The map $\phi$ defined in (3.7) and the singular part $f_{\text {sing }}$ are $G$-equivariant, too, for similar reasons.

Thus, in summary, we arrive at the following equivariant normal form theorem. 
Theorem 4.6 (Equivariant normal form - General) Let $f: M \rightarrow N$ be an equivariant map between Fréchet $G$-manifolds. Choose $m \in M$ and set $\mu=f(m)$. Assume that the following conditions hold:

1. The stabilizer subgroup $G_{\mu}$ of $\mu$ is a Lie subgroup of $G$.

2. The induced $G_{\mu}$-action on $M$ is proper and admits a slice $S$ at $m$.

3. The induced $G_{m}$-action on $N$ can be linearized at $\mu$.

4. The restriction $f^{S} \equiv f_{\uparrow S}: S \rightarrow N$ off to $S$ can be brought into a normal form at $m$ using either Theorems 3.5, 3.7, 3.8, 3.11, 3.12 and 3.14.

Then, $f$ can be brought into an equivariant normal form at $m$ relative to the $G$-action.

Proof Since the $G$-action on $M$ is proper, the stabilizer $G_{m}$ is compact. By the slice property $\mathrm{SL}_{5}$, the $G_{m}$-action on the slice $S$ can be linearized at $m$. Hence, Proposition 4.5 implies that $f^{S}$ can be brought into an equivariant normal form relative to the $G_{m}$-action. The claim now follows from Proposition 4.3.

We now comment on the assumptions of Theorem 4.6.

1. In finite dimensions, every closed subgroup of a Lie group is a Lie subgroup. This shows, in particular, that stabilizer subgroups are Lie subgroups. However, it is not known, even for Banach Lie group actions, whether the stabilizer is always a Lie subgroup, see [57, Problem IX.3.b].

2. According to Palais [62], proper actions of finite-dimensional Lie groups on finite-dimensional manifolds always admit slices. In infinite dimensions, this may no longer be true and additional assumptions have to be made. We refer to [16, 78] for general slice theorems in infinite dimensions and $[2,7,21]$ for constructions of slices in concrete examples.

3. By Bochner's linearization theorem [6, Theorem 1], every action of a compact Lie group on a finite-dimensional manifold can be linearized near a fixed point of the action. This result generalizes to actions on Banach manifolds, see [13, Proposition 3.1.4]. However, the proof of Bochner's linearization theorem relies on the inverse function theorem and so does not generalize to actions on Fréchet or more general locally convex manifolds.

4. The proof of Proposition 4.5 shows that one can use every normal form theorem provided it is equivariant with respect to linear actions of a compact group.

Remark 4.7 (Slice mapping) Recall from the proof of Proposition 4.3 the diffeomorphism $\kappa: S \rightarrow U \subseteq X$ which brings $f^{S}$ into a normal form. When $S$ is viewed as a submanifold of $M$, then the map $\Psi=\kappa^{-1}$ satisfies $\Psi(0)=m$,

$$
\mathrm{T}_{\Psi(x)} M=\mathfrak{g} \cdot \Psi(x)+\operatorname{Im} \mathrm{T}_{x} \Psi
$$

for all $x \in U$, and

$$
\mathrm{T}_{m} f \circ \mathrm{T}_{0} \Psi=\hat{f} \circ \operatorname{pr}_{\mathrm{Coim}_{m} f^{s}} .
$$

In the context when $f$ is the momentum map for a symplectic $G$-action, a map with these properties is called a slice map in [9, Definition 2.1]. The construction in [9, Proposition 2.2] of such a slice map makes no use of slices. Thus, our approach has the advantage of bringing the $G$-action into a normal form simultaneously. 


\subsection{Normal form theorem with finite-dimensional target or domain}

In the remainder of this section, we present variations of the equivariant normal form theorem 4.6 using assumptions that are often easier to verify in applications. Similar to the discussion in Sect. 3, we start with the simplest case and then work through various levels of functional-analytic settings, finishing with the tame Nash-Moser category. All assumptions of Theorem 4.6 are automatically verified in finite dimensions.

Theorem 4.8 (Equivariant normal form-finite dimensions) Let $G$ be a finite-dimensional Lie group and let $f: M \rightarrow N$ be a smooth G-equivariant map between finite-dimensional $G$-manifolds. If the $G$-action on $M$ is proper, then $f$ can be brought into an equivariant normal form at every point.

Proof Let $m \in M$ and $\mu=f(m)$. Since the stabilizer $G_{\mu}$ of $\mu$ is a closed subgroup of a finite-dimensional Lie group, it is a Lie subgroup. The induced $G_{\mu}$-action on $M$ is proper and thus the slice theorem of Palais [62] implies that there exists a slice $S$ for the $G_{\mu}$-action at $m$. Properness of the action also implies that $G_{m}$ is compact and thus Bochner's linearization theorem [6], Theorem 1; [20], Theorem 2.2.1] shows that the $G_{m}$-action on $N$ can be linearized around the fixed point $\mu$. By Theorem 3.5, $f^{S}=f_{\uparrow S}: S \rightarrow N$ can be brought into a normal form at $m$. Hence, all assumptions of Theorem 4.6 are verified and so $f$ can be brought into an equivariant normal form.

This result for the finite-dimensional case can be directly generalized to the case where only one of the manifolds involved is finite-dimensional.

Theorem 4.9 (Equivariant normal form-finite-dimensional target) Let $G$ be a Lie group and let $f: M \rightarrow N$ be a smooth G-equivariant map between $G$-manifolds, where $M$ is a Fréchet manifold and $N$ is a finite-dimensional manifold. Let $m \in M$ and $\mu=f(m)$. If the stabilizer subgroup $G_{\mu}$ of $\mu$ is a Lie subgroup of $G$ and the induced $G_{\mu}$-action on $M$ is proper and admits a slice at $m$, then $f$ can be brought into an equivariant normal form at $m$.

Proof The proof is similar to the one of Theorem 4.8 using Theorem 3.7 to bring $f^{S}$ into a normal form. We leave the details to the reader.

Theorem 4.10 (Equivariant normal form-finite-dimensional domain) Let $G$ be a finitedimensional Lie group and let $f: M \rightarrow N$ be a smooth $G$-equivariant map between $G$-manifolds, where $M$ is finite-dimensional. Let $m \in M$ and $\mu=f(m)$. If the $G$-action on $M$ is proper and the induced $G_{m}$-action on $N$ can be linearized at $\mu$, then $f$ can be brought into an equivariant normal form at $m$.

Proof The proof is similar to the one of Theorem 4.8 using Theorem 3.8 to bring $f^{S}$ into a normal form. We leave the details to the reader.

\subsection{Tame Fréchet and elliptic normal form theorem}

Let us now come to a version of Theorem 4.6 which lives in the tame Fréchet category. 
Theorem 4.11 (Equivariant normal form-Tame Fréchet) Let $G$ be a tame Fréchet Lie group and let $f: M \rightarrow N$ be an equivariant map between tame Fréchet G-manifolds. Choose $m \in M$ and set $\mu=f(m)$. Assume that the following conditions hold:

1. The stabilizer subgroup $G_{\mu}$ of $\mu$ is a tame Fréchet Lie subgroup of $G$.

2. The induced $G_{\mu}$-action on $M$ is proper and admits a tame slice $S$ at $m$.

3. The induced $G_{m}$-action on $N$ can be linearized at $\mu$.

4. The chain

$$
0 \longrightarrow \mathfrak{g}_{\mu} \longrightarrow \mathrm{T}_{s} M \stackrel{\mathrm{T}_{s} f}{\longrightarrow} \mathrm{T}_{f(s)} N \longrightarrow 0
$$

of linear maps parametrized by $s \in S$ is uniformly ${ }^{6}$ tame regular at $m$. Here, the first map is the Lie algebra action given by $\xi \mapsto \xi . s$ for $\xi \in \mathfrak{g}_{\mu}$. Then, $f$ can be brought into an equivariant normal form at $m$.

Proof Let $S$ be a tame $G_{\mu}$-slice at $m$ modeled on the tame Fréchet space $X$. According to Theorem 4.6, we have to show that the map $f^{S}: S \rightarrow N$ satisfies the hypotheses of Theorem 3.11. For this purpose, consider slice coordinates as in $\mathrm{SL}_{3}$ of Definition B.1. That is, let $\chi: W \rightarrow G_{\mu}$ be a local section of $G_{\mu} \rightarrow G_{\mu} / G_{m}$ defined on an open neighborhood $W$ of the identity coset $[e]$ such that the map $\chi^{S}: W \times S \rightarrow M$ defined by $\chi^{S}([g], s)=\chi([g]) \cdot s$ is a diffeomorphism onto its image. Clearly, $\mathrm{T}_{[e]} \chi: \mathfrak{g}_{\mu} / \mathfrak{g}_{m} \rightarrow \mathfrak{g}_{\mu}$ is a continuous right inverse of the projection $\mathfrak{g}_{\mu} \rightarrow \mathfrak{g}_{\mu} / \mathfrak{g}_{m}$ and thus induces a topological isomorphism $\mathfrak{g}_{\mu} \simeq\left(\mathfrak{g}_{\mu} / \mathfrak{g}_{m}\right) \times \mathfrak{g}_{m}$. With respect to this decomposition, we write elements $\xi \in \mathfrak{g}_{\mu}$ as pairs $\left([\xi], \xi_{\mathfrak{g}_{m}}\right)$ with $[\xi] \in \mathfrak{g}_{\mu} / \mathfrak{g}_{m}$ and $\xi_{\mathfrak{g}_{m}} \in \mathfrak{g}_{m}$. Let $\boldsymbol{l}_{S}: X \supseteq U \rightarrow S$ be a chart of $S$ which linearizes the $G_{m}$-action as in $\mathrm{SL}_{5}$, and let $\rho: N \supseteq V^{\prime} \rightarrow V \subseteq Y$ be a chart at $\mu$ which linearizes the $G_{m}$-action on $N$. We denote the chart representation of $f^{S}: S \rightarrow N$ by $f_{\rho}^{S}: X \supseteq U \rightarrow V \subseteq Y$. With respect to the local trivialization induced by $\chi^{S} \circ\left(\operatorname{id}_{W} \times l_{S}\right)$ and $\rho$, the chain (4.14) yields a family of chains

$$
0 \longrightarrow \mathfrak{g}_{\mu} \stackrel{\Gamma_{x}}{\longrightarrow} \mathfrak{g}_{\mu} / \mathfrak{g}_{m} \times X \stackrel{\Xi_{x}}{\longrightarrow} Y \longrightarrow 0
$$

parametrized by $x \in U$, where the tame smooth families of linear maps $\Gamma: U \times \mathfrak{g}_{\mu} \rightarrow \mathfrak{g}_{\mu} / \mathfrak{g}_{m} \times X$ and $\Xi: U \times \mathfrak{g}_{\mu} / \mathfrak{g}_{m} \times X \rightarrow Y$ are defined by

$$
\Gamma_{x}(\xi)=\left([\xi], \xi_{\mathfrak{g}_{m}} \cdot x\right),
$$

for $\xi \in \mathfrak{g}_{\mu}$, and

$$
\Xi_{x}([\xi], v)=\mathrm{T}_{f\left(l_{S}(x)\right)} \rho\left(\left(\mathrm{T}_{[e]} \chi([\xi])\right) \cdot f\left(l_{S}(x)\right)\right)+\mathrm{T}_{x} f_{\rho}^{S}(v)
$$

for $[\xi] \in \mathfrak{g}_{\mu} / \mathfrak{g}_{m}$ and $v \in X$. Since the chain (4.14) is uniformly tame regular at $m$, we can assume that the chain (4.15) is uniformly tame regular at $x=0$. Note that $\operatorname{Im} \Gamma_{0}=\mathfrak{g}_{\mu} / \mathfrak{g}_{m} \times\{0\}$. Thus, Proposition 2.14 implies that the family

\footnotetext{
6 To be precise, the chain should be viewed as a chain of vector bundle morphisms over $S$. Similarly to Definition 3.10, uniform regularity is then defined relative to vector bundle trivializations by requiring that the associated family of linear chains is uniformly regular in the sense of Definition 2.13.
} 


$$
\left(\Xi_{x}\right)_{\{\{[0]\} \times X}=\mathrm{T}_{x} f_{\rho}^{S}: X \mapsto Y
$$

of linear maps parametrized by $x \in U$ is uniformly tame regular at 0 . Thus, by Theorem $3.11, f^{S}$ can be brought into a normal form, which completes the proof.

Remark 4.12 In Sect. 3, we have seen that a smooth map can be brought into a (tame) normal form if and only if its differential is uniformly (tame) regular. It is perceivable that a similar equivalence holds in the equivariant setting as well. Indeed, uniform regularity of the left arm of (4.14) should imply that the action admits a near slice in the sense of [62, Definition 2.1.6], which is a weaker notion of a slice only requiring the slice coordinates $\mathrm{SL}_{3}$. The above arguments then should show that the restriction of the map to the near slice can be brought into a normal form. Working out the details of this idea is left for future work.

We emphasize that the additional topological properties distinguishing a slice from a near slice will become important in the study of moduli spaces in Sect. 5, see Remark 5.5. If one is not interested in these topological features (for example, because the focus lies on rigidity/stability phenomena), then only requiring the tame uniform regularity of the deformation complex is a promising approach. We refer the reader to [35, Section III.3.1] for work in this direction which is concerned with the case where the deformation complex has trivial homology.

For the following elliptic version of the equivariant normal form theorem, the reader might want to recall the notion of a geometric Fréchet manifold from Definition 3.13. In particular, this theorem applies to nonlinear differential operators that are equivariant under groups of diffeomorphisms or gauge transformations.

Theorem 4.13 (Equivariant normal form - elliptic) Let $G$ be a tame Fréchet Lie group and let $f: M \rightarrow N$ be an equivariant map between tame Fréchet $G$-manifolds. Let $m \in M$ and $\mu=f(m)$. Assume that the following conditions hold:

1. The stabilizer subgroup $G_{\mu}$ of $\mu$ is a geometric tame Fréchet Lie subgroup of $G$.

2. The induced $G_{\mu}$-action on $M$ is proper and admits a geometric slice $S$ at $m$.

3. The induced $G_{m}$-action on $N$ can be linearized at $\mu$.

4. The chain

$$
0 \longrightarrow \mathfrak{g}_{\mu} \longrightarrow \mathrm{T}_{s} M \stackrel{\mathrm{T}_{s} f}{\longrightarrow} \mathrm{T}_{f(s)} N \longrightarrow 0
$$

is a chain of geometric linear maps parametrized by $s \in S$, which is an elliptic complex at $m$.

Then, $f$ can be brought into an equivariant normal form at $m$.

Proof The claim follows directly as a special case of Theorem 4.11, because, according to Theorem 2.15, a tame family of chains of differential operators is uniformly tame regular in a neighborhood of a point at which the chain is an elliptic complex. 


\section{Moduli spaces}

In abstract terms, a moduli space is a space whose points parametrize isomorphism classes of geometric objects. Usually, one is mainly interested in a subclass of geometric objects satisfying an additional condition, which is often phrased in the form of a partial differential equation. In the following, we will consider moduli spaces fitting into the following general setup. Let $f: M \rightarrow N$ be an equivariant map between $G$-manifolds. For every $\mu \in N$, set

$$
\check{M}_{\mu} \equiv f^{-1}(\mu) / G_{\mu},
$$

where $G_{\mu}$ is the stabilizer subgroup of $\mu$ under the $G$-action on $N$. Here, $M$ is the space of geometric objects, the equation $f(m)=\mu$ describes the additional properties of these objects one is interested in, and the $G$-action implements the notion of equivalence. Because of the flexible nature of this general setting, many well-known moduli spaces fit into this framework, see Sects. 5.3-5.5 for applications. Moreover, symplectic quotients are examples of these abstract moduli spaces, and the general theory developed in the next sections lays the foundation to generalize singular symplectic reduction to infinite dimensions, which will be presented elsewhere [13, 14].

In the simplest case, when $\mu$ is a regular value of $f$ and $G_{\mu}$ acts freely, the space $\check{M}_{\mu}$ is a smooth manifold. However, in general, the moduli space has a complicated geometry with singularities. In this section, we investigate the local structure of the moduli space $\check{M}_{\mu}=f^{-1}(\mu) / G_{\mu}$ under the assumption that $f$ can be brought into a normal form. In this case, $\check{M}_{\mu}$ can be locally identified with the quotient of the zero set of a smooth map by the linear action of a compact group, i.e., it has the structure of a Kuranishi space. In Sect. 5.2, we find additional conditions on the normal form which ensure that $\check{M}_{\mu}$ is stratified by orbit types.

\subsection{Kuranishi structures}

Kuranishi spaces were introduced by Fukaya and Ono [27, Section 1.5] in their study of the geometry of moduli spaces of pseudoholomorphic curves. The notion of a Kuranishi structure builds on ideas of Kuranishi [46] for the moduli space of complex structures and of Donaldson [18, Section II.2] and Taubes [79, Section 6] for moduli problems in gauge theory, see also [19, Section 4.2.5].

Definition 5.1 Let $\mathscr{X}$ be a topological space. A Kuranishi chart at a point $x \in \mathscr{X}$ is a tuple $(V, E, F, H, s, \kappa)$ consisting of the following data:

1. an open neighborhood $V$ of 0 in a locally convex space $E$,

2. a locally convex space $F$,

3. a compact Lie group $H$ acting linearly and continuously on $V$ and $F$,

4. a smooth $H$-equivariant map $s: V \rightarrow F$,

5. a homeomorphism $\kappa$ from $s^{-1}(0) / H$ to a neighborhood of $x$ in $\mathscr{X}$.

The bundle $F \times V \rightarrow V$ is called the obstruction bundle and $s$ is referred to as the obstruction map. If $E$ and $F$ are finite-dimensional vector spaces, then the Kuranishi chart is said to be finite-dimensional. 
Informally speaking, a space admitting Kuranishi charts is locally modeled on the quotient of the zero set of a smooth map by a compact group. For a finite-dimensional Kuranishi chart $(V, E, F, H, s, \kappa)$ at $x \in \mathscr{X}$, the number

$$
\operatorname{dim} E-\operatorname{dim} F-\operatorname{dim} H
$$

is called the virtual dimension of $\mathscr{X}$ (at $x$ ). This is motivated by the fact that $s^{-1}(0) / H$ forms a manifold of this dimension at regular points of $s$ for which the $H$-action is free.

\section{Remarks 5.2}

1. If the map $s$ vanishes and $H$ is a finite group acting faithfully on $E$, then the Kuranishi chart $(V, E, F, H, s, \kappa)$ reduces to an infinite-dimensional orbifold chart, i.e., $\mathscr{X}$ is locally modeled on the quotient of $E$ by a finite group action. If, in addition, the $H$-action on $E$ is trivial, then we obtain an ordinary manifold chart on $\mathscr{X}$.

2. Our notion of a finite-dimensional Kuranishi chart is a generalization of the one proposed by Oh et al. [60, Definition A.1.1]. There, $H$ is assumed to be a finite group (acting effectively on $V$ ). Finiteness of $H$ is a natural assumption in the context of moduli spaces of pseudoholomorphic curves, but it is too restrictive in our more general setting. Moreover, we do not require $\mathscr{X}$ to be compact nor to be endowed with a metric as it is done in [60].

Usually, only finite-dimensional Kuranishi charts are discussed in the literature. However, for general moduli spaces, one cannot expect the Kuranishi chart to be finitedimensional. As we will see below in Remark 5.4, this amounts to requiring that a certain complex is Fredholm.

3. In order to define a Kuranishi structure on $\mathscr{X}$, in a similar spirit to the smooth structure of a manifold, one needs to introduce coordinate transition maps in order to glue together different Kuranishi charts. A variety of definitions of suitable chart transitions are proposed in the literature, each with their own advantages and functorial properties. We refer the reader to [43, Appendix A] for a review on this matter.

4. Recently, Hofer, Wysocki and Zehnder have introduced the polyfold framework as a different approach to deal with the analytic and geometric issues occurring in the study of moduli spaces in symplectic field theory, see [40,41] and references therein. We refer to [81] for an extensive discussion of the relation of Kuranishi structures and polyfold theory. A detailed comparison of the tame Fréchet category with the so-called scale calculus that underlies the polyfold framework can be found in [28].

The equivariant normal form studied in the previous section is a suitable tool to obtain information about the local structure of the moduli space.

Theorem 5.3 Let $f: M \rightarrow N$ be an equivariant map between $G$-manifolds. Choose $\mu \in N$ such that $f^{-1}(\mu)$ is not empty. Assume that the stabilizer subgroup $G_{\mu}$ of $\mu$ is a Lie subgroup of $G$. If $f$ can be brought into an equivariant normal form at every point of $f^{-1}(\mu)$, then there exists a Kuranishi chart on $\check{M}_{\mu} \equiv f^{-1}(\mu) / G_{\mu}$ at every point.

Proof Let $m \in f^{-1}(\mu)$, and let $\left(G_{m}, X, Y, \hat{f}, f_{\text {sing }}\right)$ be an abstract equivariant normal form of $f$ at $m$ in the sense of Definition 4.1, with associated decompositions $X=\operatorname{Ker} \oplus \operatorname{Coim}$ and $Y=$ Coker $\oplus \operatorname{Im}$. Consider a slice $S$ at $m$ for the $G_{\mu}$-action, diffeomorphic to the domain $U \subseteq X$ of $f_{\text {sing }}$ via a $G_{m}$-equivariant diffeomorphism $l_{S}: X \supseteq U \rightarrow S$. In the following, we 
suppress the slice diffeomorphism $l_{S}$. Denote the tube diffeomorphism associated with $S$ by $\chi^{\mathrm{T}}: G_{\mu} \times_{G_{m}} U \rightarrow M$. For a $G_{m}$-equivariant chart $\rho: N \supseteq V^{\prime} \rightarrow V \subseteq Y$ at $\mu$ define $\rho^{\mathrm{T}}: G_{\mu} \times_{G_{m}} V \rightarrow N$ by $\rho^{\mathrm{T}}([g, y])=g \cdot \rho^{-1}(y)$. According to Definition 4.2, we can choose $S$ and $\rho$ so that the following diagram commutes:

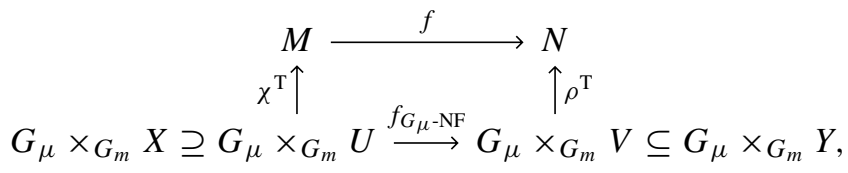

where $f_{G_{\mu}-\mathrm{NF}}$ is defined by $f_{G_{\mu}-\mathrm{NF}}\left(\left[g, x_{1}, x_{2}\right]\right)=\left[g, \hat{f}\left(x_{2}\right)+f_{\text {sing }}\left(x_{1}, x_{2}\right)\right]$ for $g \in G_{\mu}$, $x_{1} \in U \cap$ Ker and $x_{2} \in U \cap$ Coim. Note that $\left(\rho^{\mathrm{T}}\right)^{-1}(\mu)=G_{\mu} \times_{G_{m}}\{0\}$, by the definition of $\rho^{\mathrm{T}}$. Using the commutative diagram (5.3), we hence obtain

$$
\begin{aligned}
\left(\chi^{\mathrm{T}}\right)^{-1}\left(f^{-1}(\mu)\right) & =f_{G_{\mu}-\mathrm{NF}}^{-1}\left(\left(\rho^{\mathrm{T}}\right)^{-1}(\mu)\right) \\
& =f_{G_{\mu}-\mathrm{NF}}^{-1}\left(G_{\mu} \times_{G_{m}}\{0\}\right) \\
& =G_{\mu} \times_{G_{m}}\left\{\left(x_{1}, 0\right) \in U: f_{\text {sing }}\left(x_{1}, 0\right)=0\right\} .
\end{aligned}
$$

By $G_{\mu}$-equivariance, the tube diffeomorphism $\chi^{\mathrm{T}}$ thus induces a local homeomorphism of $\check{M}_{\mu}=f^{-1}(\mu) / G_{\mu}$ with

$$
\left\{\left(x_{1}, 0\right) \in U: f_{\text {sing }}\left(x_{1}, 0\right)=0\right\} / G_{m} .
$$

This local homeomorphism provides a Kuranishi chart on $\check{M}_{\mu}$ with $E=\operatorname{Ker}, V=U \cap \operatorname{Ker}$, $F=$ Coker, $H=G_{m}$ and $s=f_{\text {sing }}(\cdot, 0): V \rightarrow F$ in the notation of Definition 5.1.

Remark 5.4 In the setting of Theorem 5.3, consider the chain complex

$$
0 \longrightarrow \mathfrak{g}_{\mu} \stackrel{\mathrm{T}_{e} \Upsilon_{m}}{\longrightarrow} \mathrm{T}_{m} M \stackrel{\mathrm{T}_{m} f}{\longrightarrow} \mathrm{T}_{\mu} N \longrightarrow 0,
$$

where $\Upsilon_{m}: G_{\mu} \rightarrow M$ is the orbit map of the $G_{\mu}$-action at $m \in M$. Recall from (4.6) that the homology groups of this complex are identified with the abstract spaces

$$
\mathfrak{g}_{m} \simeq \mathfrak{h}, \quad \operatorname{KerT}_{m} f / \mathfrak{g}_{\mu} \cdot m \simeq \text { Ker }, \quad \mathrm{T}_{\mu} N / \operatorname{Im~T}_{m} f \simeq \text { Coker }
$$

occurring in the equivariant normal form. For the Kuranishi charts on $\check{M}_{\mu}$ constructed in the proof of Theorem 5.3, we have $H=G_{m}, E=$ Ker and $F=$ Coker. Thus, these Kuranishi charts are finite-dimensional if and only if the complex (5.6) is Fredholm.

In this case, the Euler characteristic of the complex (5.6),

$$
\operatorname{dim} \mathfrak{g}_{m}-\operatorname{dim} \text { Ker }+\operatorname{dim} \text { Coker, }
$$

is (minus) the virtual dimension of $\check{M}_{\mu}$.

Remark 5.5 The proof of Theorem 5.3 relies in an essential way on the fact that the tube map $\chi^{\mathrm{T}}: G_{\mu} \times_{G_{m}} S \rightarrow M$ associated with the slice $S$ is a diffeomorphism onto an open $G_{\mu}$ -invariant neighborhood of the orbit $G \cdot m$. This is also the first instance where we use injectivity of $\chi^{\mathrm{T}}$, i.e., slice property $\mathrm{SL}_{2}$. A close inspection of the proof of Theorem 5.3 shows that for the Kuranishi structure on $\check{M}_{\mu}$ one actually needs only the restriction of $\chi^{\mathrm{T}}$ 
to $G_{\mu} \times_{G_{m}}\left(S \cap f^{-1}(\mu)\right)$ to be injective. This is equivalent to the following weaker version of $\mathrm{SL}_{2}$ :

$\left(\mathrm{SL} 2_{\mu}\right) \quad$ Any $g \in G$ for which $g \cdot\left(S \cap f^{-1}(\mu)\right)$ intersects non-trivially with $S \cap f^{-1}(\mu)$ is necessarily an element of the stabilizer $G_{m}$.

Recall that the slice property $\mathrm{SL}_{2}$ is usually established using some form of compactness of the group action, e.g., properness. Thus, for $\left(\mathrm{SL} 2_{\mu}\right)$, one needs such a compactness property only on the solution set $f^{-1}(\mu)$. This observation enables one to use compactness arguments specific to the partial differential equation under study. We will encounter such a case for pseudoholomorphic curves where Gromov's compactness theorem ensures that $\left(\mathrm{SL}_{\mu}\right)$ holds although $\mathrm{SL}_{2}$ is not satisfied in general, see Sect. 5.5.

Moreover, we so far did not make any use of property $\mathrm{SL}_{4}$ which will only play a fundamental role for the orbit-type stratification discussed in Sect. 5.2 below. Thus, we expect that a version of Theorem 5.3 also holds in the more general setting, where one only requires the weak equivariant normal form that uses a near slice instead of a slice, as discussed in Remark 4.12, and the weak slice property $\left(\mathrm{SL}_{\mu}\right)$.

Since every equivariant map between finite-dimensional $G$-manifolds can be brought into an equivariant normal form according to Theorem 4.8, we obtain the following corollary of Theorem 5.3.

Corollary 5.6 Let $f: M \rightarrow N$ be an equivariant map between finite-dimensional G-manifolds and let $\mu \in N$. If the $G_{\mu}$-action on $M$ is proper, then $\check{M}_{\mu} \equiv f^{-1}(\mu) / G_{\mu}$ admits a Kuranishi chart at every point.

Of course, the equivariant normal form theorems 4.9, 4.10, 4.11 and 4.13 give similar corollaries in infinite dimensions.

\subsection{Orbit-type stratification}

As we have seen, the abstract moduli space $\check{M}_{\mu}=f^{-1}(\mu) / G_{\mu}$ admits Kuranishi charts if $f$ can be brought into an equivariant normal form. Under additional conditions on the equivariant normal form, $\check{M}_{\mu}$ has an even more pleasant structure. For the following result, the reader might want to recall the notions of orbit type and stratification from Appendix B. Roughly speaking, a stratification is a decomposition of a space such that the pieces are manifolds and a certain approximation condition holds when one approaches the boundary of a piece. These properties have a local nature and, in fact, can be ensured by requiring the equivariant normal form to satisfy additional conditions.

Proposition 5.7 Let $f: M \rightarrow N$ be a smooth equivariant map between $G$-manifolds, and let $\mu \in N$. Assume that the stabilizer subgroup $G_{\mu}$ is a Lie subgroup of $G$ and that the induced action of $G_{\mu}$ on $M$ is proper. Moreover, assume that $f$ can be brought into an equivariant normal form $\left(G_{m}, X, Y, \hat{f}, f_{\text {sing }}\right)$ at every $m \in f^{-1}(\mu)$ such that the following holds: 
1. (Submanifold property) The set

$$
\left\{\left(x_{1}, 0\right) \in U_{\left(G_{m}\right)}: f_{\text {sing }}\left(x_{1}, 0\right)=0\right\}=f_{\text {sing }}^{-1}(0) \cap \operatorname{Ker} \cap X_{\left(G_{m}\right)}
$$

is a submanifold of $X_{\left(G_{m}\right)}$.

2. (Approximation property) For every orbit type $(K) \leq\left(G_{m}\right)$ of the $G_{\mu}$-action on $M$, the point 0 lies in the closure of $f_{\text {sing }}^{-1}(0) \cap \operatorname{Ker} \cap X_{(K)}$ in $X$.

Then, the partition of $f^{-1}(\mu)$ into the orbit-type subsets $f^{-1}(\mu) \cap M_{(H)}$ is a stratification. Moreover, the decomposition of $\check{M}_{\mu}=f^{-1}(\mu) / G_{\mu}$ into the $\operatorname{sets}\left(f^{-1}(\mu) \cap M_{(H)}\right) / G_{\mu}$ is a stratification, too.

Proof Let $m \in f^{-1}(\mu)$ and let $\left(G_{m}, X, Y, \hat{f}, f_{\text {sing }}\right)$ be an equivariant normal form of $f$ at $m$ satisfying the submanifold and approximation properties. In the proof of Theorem 5.3, we have seen that the $G_{\mu}$-equivariant tube diffeomorphism $\chi^{\mathrm{T}}$ identifies the level set $f^{-1}(\mu)$ locally with

$$
G_{\mu} \times_{G_{m}}\left\{\left(x_{1}, 0\right) \in U: f_{\text {sing }}\left(x_{1}, 0\right)=0\right\} .
$$

Accordingly, $f^{-1}(\mu) \cap S_{\left(G_{m}\right)}$ is identified with the set

$$
\left\{\left(x_{1}, 0\right) \in U_{\left(G_{m}\right)}: f_{\text {sing }}\left(x_{1}, 0\right)=0\right\} .
$$

Since the latter is a submanifold of $X_{\left(G_{m}\right)}$ by the submanifold property, we conclude that $f^{-1}(\mu) \cap S_{\left(G_{m}\right)}$ is a closed submanifold of $S_{\left(G_{m}\right)}$. Moreover, the approximation property entails that, for every $G_{\mu}$-orbit type $(K) \leq\left(G_{m}\right)$, the point $m$ lies in the closure of $f^{-1}(\mu) \cap S_{(K)}$. Thus, the claims follow from Proposition B.4.

\subsection{Application: moduli space of anti-self-dual connections}

The local structure of the moduli space of anti-self-dual Yang-Mills connections is well understood, see, e.g., [19]. We will show how these results can be rederived with relatively small effort using the general framework developed in the previous sections.

Consider a principal $G$-bundle $\pi: P \rightarrow M$ over a compact $n$-dimensional Riemannian manifold $M$ with $G$ being a compact Lie group. A connection in $P$ is a $G$-equivariant splitting of the tangent bundle $\mathrm{T} P=\mathrm{V} P \oplus \mathrm{H} P$ into the canonical vertical distribution $\mathrm{V} P$ and a horizontal distribution $\mathrm{H} P$. Equivalently, a connection $A$ in $P$ yields a splitting of the Atiyah sequence

$$
0 \longrightarrow \mathrm{Ad} P \longrightarrow \mathrm{T} P / G \stackrel{\mathrm{T} \pi}{\longrightarrow} \mathrm{T} M \longrightarrow 0 .
$$

Accordingly, the space $\mathcal{C}(P)$ of connections on $P$ is identified with the space of sections of an affine bundle over $M$, and in this way $\mathcal{C}(P)$ carries a natural tame Fréchet manifold structure modeled on the vector space $\Omega^{1}(M, \operatorname{Ad} P)$, cf. [1]. The covariant derivative and the curvature of a connection $A$ are denoted by $\mathrm{d}_{A}$ and $F_{A}$, respectively.

The group $\mathcal{G}(P)$ of local gauge transformations on $P$ is a tame Fréchet Lie group, because it is realized as the space of smooth sections of the group bundle $P \times_{G} G$, see [10] for details. The natural left action of $\mathcal{G}(P)$ on $\mathcal{C}(P)$ is given by 


$$
A \mapsto \operatorname{Ad}_{\lambda} A+\lambda \mathrm{d} \lambda^{-1}
$$

for $\lambda \in \mathcal{G}(P)$. This action is proper, see [12, 68]. Moreover, it admits a slice $\mathcal{S}_{A_{0}}$ at every $A_{0} \in \mathcal{C}$ given by the Coulomb gauge condition. That is, ${ }^{7}$

$$
\mathcal{S}_{A_{0}}:=\left\{A \in \mathcal{U}: \mathrm{d}_{A_{0}}^{*}\left(A-A_{0}\right)=0\right\},
$$

where $\mathcal{U}$ is a suitable open neighborhood of $A_{0}$ in $\mathcal{C}(P)$. One uses the Nash-Moser inverse function theorem to show that $\mathcal{S}_{A_{0}}$ is a slice. The details can be found in $[2,12]$. In the Banach context, the orbit-type decomposition of $\mathcal{C}(P)$ has been extensively studied in [44], see also [68]. The proof that the decomposition satisfies the frontier condition [44, Theorem 4.3.5] carries over to our Fréchet setting without major changes. As a consequence of Proposition B.4, the decomposition of $\mathcal{C}(P)$ into gauge orbit types is a stratification. Finally, the gauge orbit types are in bijection with the set of isomorphism classes of holonomyinduced Howe subbundles of $P$, cf. [68, Theorem 3.3]. Using this observation, the classification of orbit types for all classical groups has been accomplished in [37, 38, 67-69].

Coming to the anti-self-dual equation, we specialize to the case where $M$ is an oriented compact Riemannian manifold of dimension 4 and $G$ is a compact, semisimple Lie group. On a four-dimensional manifold, the Hodge star operator $*$ associated with the Riemannian metric satisfies $* *=\mathrm{id}$ on 2 -forms and thus determines a decomposition

$$
\Omega^{2}(M)=\Omega_{+}^{2}(M) \oplus \Omega_{-}^{2}(M)
$$

of the space of 2-forms into the \pm 1 -eigenspaces. A similar decomposition holds for vectorvalued 2-forms and, in particular, the curvature $F_{A}$ of a connection $A \in \mathcal{C}(P)$ can be written as

$$
F_{A}=F_{A}^{+}+F_{A}^{-}
$$

with $F_{A}^{ \pm} \in \Omega_{ \pm}^{2}(M, \operatorname{Ad} P)$. A connection $A$ with $F_{A}^{+}=0$ is called anti-self-dual (ASD). The Bianchi identity implies that an ASD connection satisfies the Yang-Mills equation. The self-dual part of the curvature gives a smooth map

$$
F^{+}: \mathcal{C}(P) \rightarrow \Omega_{+}^{2}(M, \operatorname{Ad} P)
$$

which is equivariant with respect to the natural actions of $\mathcal{G}(P)$. The moduli space of antiself-dual connections is, by definition, the space

$$
\mathcal{A}=\left(F^{+}\right)^{-1}(0) / \mathcal{G}(P)
$$

of anti-self-dual connections on $P$ modulo gauge equivalence. This clearly fits into the general framework considered in the previous sections. Let us verify the conditions of Theorem 4.13:

1. The stabilizer of $\mu=0 \in \Omega_{+}^{2}(M, \operatorname{Ad} P)$ is the whole group $\mathcal{G}(P)$, which is a geometric tame Fréchet Lie group with Lie algebra $\mathrm{LG}(P)=\Gamma^{\infty}(\operatorname{Ad} P)$.

2. The natural action of $\mathcal{G}(P)$ on $\mathcal{C}(P)$ is proper and admits slices as discussed above.

3. The action of $\mathcal{G}(P)$ on $\Omega_{+}^{2}(M, \operatorname{Ad} P)$ is clearly linear.

\footnotetext{
${ }^{7}$ Here, as usual, $\mathrm{d}_{A}^{*} \alpha:=(-1)^{k} * \mathrm{~d}_{A} * \alpha$ for a $k$-form $\alpha$.
} 
4. Let $A$ be an ASD connection and let $\mathcal{S}$ be a slice at $A$. The chain (4.14) here takes the form

$$
0 \longrightarrow \Omega^{0}(M, \operatorname{Ad} P) \stackrel{\mathrm{d}_{B}}{\longrightarrow} \Omega^{1}(M, \operatorname{Ad} P) \stackrel{\mathrm{d}_{B}^{+}}{\longrightarrow} \Omega_{+}^{2}(M, \operatorname{Ad} P) \longrightarrow 0,
$$

where $B \in \mathcal{S}$ and $\mathrm{d}_{B}^{+}$denotes the self-dual part of the covariant derivative $\mathrm{d}_{B}$. This chain is clearly a chain of linear differential operators tamely parametrized by the connection $B$. The ASD condition for $A$ asserts that $\mathrm{d}_{A}^{+} \circ \mathrm{d}_{A}=0$ and so, at $B=A$, the chain (5.19) is a complex, called the Yang-Mills complex. This complex has been studied first by Atiyah et al. [5]. Ellipticity of the Yang-Mills complex is well known and follows from a straightforward computation in linear algebra, see, e.g., [70, Lemma 6.5.2]. Moreover, applying the Atiyah-Singer index theorem one finds the following for its Euler characteristic:

$$
-2 \mathrm{p}_{1}(\mathrm{Ad} P)+\frac{1}{2}\left(\chi_{M}-\sigma_{M}\right) \cdot \operatorname{dim} G,
$$

where $\mathrm{p}_{1}(\operatorname{Ad} P)$ is the Pontryagin index of the adjoint bundle, $\chi_{M}$ is the Euler number of $M$ and $\sigma_{M}$ is the signature of $M$, see [5, p. 446] and [70, Lemma 6.5.5] for a detailed proof.

Hence, by Theorem 4.13, the map $F^{+}$can be brought into an equivariant normal form at every ASD connection $A \in \mathcal{C}(P)$. Moreover, as a consequence of Theorem 5.3 and Remark 5.4, we obtain the following description of the local geometry of the moduli space of anti-self-dual connections.

Theorem 5.8 Let $P$ be a principal G-bundle with a compact, semisimple structure group $G$ over a four-dimensional compact Riemannian manifold $M$. Then, the moduli space $\mathcal{A}$ of anti-self-dual connections on $P$ admits a finite-dimensional Kuranishi chart at every point. Moreover, the virtual dimension of $\mathcal{A}$ is given by

$$
2 \mathrm{p}_{1}(\operatorname{Ad} P)-\frac{1}{2}\left(\chi_{M}-\sigma_{M}\right) \cdot \operatorname{dim} G
$$

Let us describe the constructed Kuranishi charts on $\mathcal{A}$ in more detail. For this purpose, let $A \in \mathcal{C}(P)$ be an ASD connection. According to Remark 5.4, the linear spaces occurring in definition of a Kuranishi chart at $[A] \in \mathcal{A}$ are given by the cohomology groups

$$
\begin{aligned}
& \mathcal{E}=\mathrm{H}_{A}^{1,+}(M, \operatorname{Ad} P) \equiv \operatorname{Kerd}_{A}^{+} / \operatorname{Imd}_{A}, \\
& \mathcal{F}=\mathrm{H}_{A}^{2,+}(M, \operatorname{Ad} P) \equiv \Omega_{+}^{2}(M, \operatorname{Ad} P) / \operatorname{Imd}_{A}^{+} .
\end{aligned}
$$

These spaces are finite-dimensional, because the Yang-Mills complex is elliptic. Moreover, they are endowed with a natural linear action of the compact stabilizer subgroup $\mathcal{G}_{A}(P)$ of $A$. Thus, the obstruction map is a $\mathcal{G}_{A}(P)$-equivariant map

$$
\text { s: } \mathrm{H}_{A}^{1,+}(M, \operatorname{Ad} P) \supseteq \mathcal{V} \rightarrow \mathrm{H}_{A}^{2,+}(M, \operatorname{Ad} P),
$$


where $\mathcal{V}$ is a $\mathcal{G}_{A}(P)$-invariant, open neighborhood of 0 in $\mathrm{H}_{A}^{1,+}(M, \operatorname{Ad} P)$. Finally, the moduli space $\mathcal{A}$ in a neighborhood of $[A]$ is modeled on the quotient $s^{-1}(0) / \mathcal{G}_{A}(P)$. In this way, we recover in our framework the well-known result [19, Proposition 4.2.23] concerning the local structure of $\mathcal{A}$.

For completeness, we include some remarks on how to get further insights into the geometry of $\mathcal{A}$. This requires a more precise control of the obstruction map $s$, which is rather difficult to obtain in full generality. However, in concrete examples, one can often find conditions which ensure that $s$ vanishes. For example, if $M$ is a compact selfdual Riemannian manifold with positive scalar curvature, then it can be shown using the Weitzenböck formula that $\mathrm{H}_{A}^{2,+}(M, \operatorname{Ad} P)$ is trivial for every irreducible ASD connection $A$. Thus, in this case, the moduli space of irreducible anti-self-dual connections is either empty or a smooth manifold. This important result was originally obtained by Atiyah, Hitchin and Singer [5, Theorem 6.1].

For the remainder, we specialize to $G=\mathrm{SU}(2)$. This setting was used by Donaldson [18] to gain astounding insights into the topology and geometry of 4-manifolds. For $G=\mathrm{SU}(2)$, we have $\mathrm{p}_{1}(\operatorname{Ad} P)=4 k$, where $k$ is the so-called instanton number. Let us restrict our attention to the case $k=1$. Moreover, let us assume that $M$ is simply connected and that its intersection form is positive definite. These assumptions imply $\chi_{M}-\sigma_{M}=1$ so that the virtual dimension of $\mathcal{A}$ is $8-3=5$. For $G=\mathrm{SU}(2)$, a reducible connection $A$ has a stabilizer group conjugate to $\mathrm{U}(1)$ or to $\mathbb{Z}_{2}$. Connections with a discrete stabilizer subgroup are flat as a consequence of the Ambrose-Singer theorem and thus only connections with a stabilizer subgroup conjugate to U(1) are of interest for the geometry of $\mathcal{A}$. One can show that there are only finitely many gauge-equivalence classes of ASD connections which are reducible to U(1). The Kuranishi charts constructed above determine the structure of $\mathcal{A}$ in a neighborhood of a singular point. Let $A$ be an ASD connection that is reducible to U(1). In the present setting, a straightforward application of the representation theory of U(1) yields the following isomorphisms of $\mathcal{G}_{A}(P)$-representation spaces

$$
\mathrm{H}_{A}^{1,+}(M, \operatorname{Ad} P) \simeq \mathbb{C}^{p}, \quad \mathrm{H}_{A}^{2,+}(M, \operatorname{Ad} P) \simeq \mathbb{C}^{q}
$$

for some $p$ and $q$ satisfying $p+q=3$, where $\mathrm{U}(1)$ acts in the usual way on $\mathbb{C}^{p}$ and $\mathbb{C}^{q}$, see [24, Proposition 4.9]. Thus, if $\mathrm{H}_{A}^{2,+}(M, \mathrm{Ad} P)$ is trivial, then, in a neighborhood of the singular point $[A]$, the moduli space $\mathcal{A}$ is identified with the cone $\mathbb{C}^{3} / \mathrm{U}(1)$ over $\mathbb{C P}^{2}$. Thus, combining Theorem 5.8 with these additional insights, one recovers the important result of Donaldson [18]. The case of a non-trivial cohomology group $\mathrm{H}_{A}^{2,+}(M, \mathrm{Ad} P)$ is more complicated and a perturbation of the metric on $M$ is required, see [24, Theorem 4.19].

The concrete description of the singularities shows that every singular connection in $\mathcal{A}$ can be approximated by a sequence of irreducible ASD connections. This implies that $\mathcal{A}$ is stratified by orbit types. In fact, for all examples known to us, the moduli space of antiself-dual connections turns out to be stratified by orbit types. We do not know whether this holds true in general.

\subsection{Application: Seiberg-Witten moduli space}

This section is concerned with the Seiberg-Witten moduli space first studied in [73, 74]. We show how our general framework recovers the known local structure of this moduli space. Our presentation follows the textbook [58] in conventions and notation. 
For the convenience of the reader, let us start by recalling basic notions from spin geometry. For $n \geq 3$ even, denote the connected double cover of $\operatorname{SO}(n)$ by $\operatorname{Spin}(n)$, which is a principal bundle pr: $\operatorname{Spin}(n) \rightarrow \operatorname{SO}(n)$ with structure group $\pi_{1} \operatorname{SO}(n) \simeq \mathbb{Z}_{2}$. We can thus form $\operatorname{Spin}^{\mathbb{C}}(n)=\operatorname{Spin}(n) \times_{\mathbb{Z}_{2}} \mathrm{U}(1)$, where $\mathbb{Z}_{2}$ acts by multiplication with -1 on $\mathrm{U}(1)$. The irreducible half-spin representations ${ }^{8} \gamma_{ \pm}: \operatorname{Spin}(n) \rightarrow \mathrm{U}\left(\Delta_{n}^{ \pm}\right)$extend to representations $\gamma_{ \pm}: \operatorname{Spin}^{\mathbb{C}}(n) \rightarrow \mathrm{U}\left(\Delta_{n}^{ \pm}\right)$of $\operatorname{Spin}^{\mathbb{C}}(n)$ defined by $\gamma_{ \pm}([g, z])(\psi)=z \cdot \gamma(g)(\psi)$. Moreover, the group homomorphism $\lambda: \operatorname{Spin}^{\mathbb{C}}(n) \rightarrow \mathrm{U}(1)$ given by $\lambda([g, z])=z^{2}$ fits into the following commutative diagram

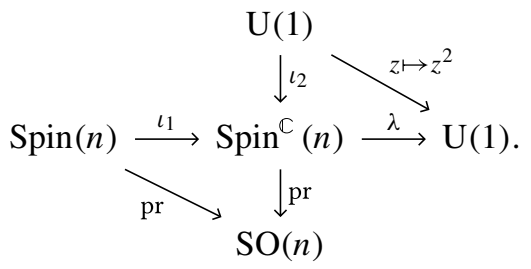

A Spin ${ }^{\mathbb{C}}$-structure on an oriented Riemannian manifold $(M, g)$ is a principal $\operatorname{Spin}^{\mathbb{C}}(n)$-bundle $\operatorname{Spin}^{\mathbb{C}} M \rightarrow M$ together with a vertical principal bundle morphism to the $\mathrm{SO}(n)$-frame bundle $\mathrm{L} M \rightarrow M$ :

$$
\begin{gathered}
\operatorname{Spin}^{\mathbb{C}} M \longrightarrow \mathrm{L} M \\
\downarrow \operatorname{Spin}^{\complement}(n) \rightarrow \operatorname{SO}(n) \downarrow \\
M \underset{\operatorname{id}_{M}}{\longrightarrow} M .
\end{gathered}
$$

The unitary spin representations $\gamma_{ \pm}$give rise to Hermitian vector bundles

$$
\Delta^{ \pm} M=\operatorname{Spin}^{\mathbb{C}} M \times_{\gamma_{ \pm}} \Delta_{n}^{ \pm},
$$

whose sections are called spinors. The Clifford multiplication $\mathbb{R}^{n} \times \Delta_{n}^{ \pm} \rightarrow \Delta_{n}^{\mp}$ is equivariant and thus yields a bundle map cl: TM $\times_{M} \Delta^{ \pm} M \rightarrow \Delta^{\mp} M$. With a slight abuse of notation, we consider $\mathrm{cl}$ to be defined on the cotangent bundle as well under the isomorphism $g^{\sharp}: \mathrm{T}^{*} M \rightarrow \mathrm{T} M$. Moreover, let

$$
P=\operatorname{Spin}^{\mathbb{C}} M \times_{\lambda} \mathrm{U}(1)
$$

be the principal $\mathrm{U}(1)$-bundle associated with the group homomorphism $\lambda$. As the maps $(\operatorname{pr}, \lambda): \operatorname{Spin}^{\mathbb{C}}(n) \rightarrow \mathrm{SO}(n) \times \mathrm{U}(1)$ induce an isomorphism on the level of Lie algebras, every connection $A$ on $P$ lifts together with the Levi-Civita connection (seen as connection on $\mathrm{L} M$ ) to a connection on $\operatorname{Spin}^{\mathbb{C}} M$. The induced covariant derivative on $\Delta^{ \pm} M$ is denoted by $\nabla_{A}$. Given a connection $A \in \mathcal{C}(P)$, the Dirac operator $\mathrm{D}_{A}$ is the composition

\footnotetext{
${ }^{8}$ For $n=4$, we have $\Delta_{4}^{ \pm} \simeq \mathbb{C}^{2}$ and the spin representation $\gamma_{ \pm}$is given by

$$
\gamma_{ \pm}: \operatorname{Spin}(4) \rightarrow \mathrm{U}(2), \quad\left(h_{+}, h_{-}\right) \mapsto h_{ \pm}
$$
}

under the isomorphism $\operatorname{Spin}(4) \simeq \mathrm{SU}(2) \times \mathrm{SU}(2)$. 


$$
\Gamma^{\infty}\left(\Delta^{+} M\right) \stackrel{\nabla_{A}}{\longrightarrow} \Gamma^{\infty}\left(\mathrm{T}^{*} M \otimes \Delta^{+} M\right) \stackrel{\mathrm{cl}}{\longrightarrow} \Gamma^{\infty}\left(\Delta^{-} M\right)
$$

In the following, $M$ is a closed oriented Riemannian manifold of dimension 4 endowed with a Spin ${ }^{\mathbb{C}}$-structure. The Seiberg-Witten equations for a connection $A \in \mathcal{C}(P)$ and a spinor $\psi \in \Gamma^{\infty}\left(\Delta^{+} M\right)$ are

$$
\begin{gathered}
\mathrm{D}_{A} \psi=0, \\
F_{A}^{+}=q(\psi)+\mu
\end{gathered}
$$

where $F_{A}^{+}$denotes the self-dual component of the curvature $F_{A} \in \Omega^{2}(M, \mathrm{i} \mathbb{R})$ of $A$ and $\mu \in \Omega_{+}^{2}(M, \mathrm{i} \mathbb{R})$ is a given self-dual 2-form. Moreover, the bundle map $q: \Delta^{+} M \rightarrow \mathrm{i} \Lambda_{+}^{2} \mathrm{~T}^{*} M$ is induced by the quadratic form $q: \Delta_{4}^{+} \rightarrow \mathrm{i} \Lambda_{+}^{2} \mathbb{R}^{4}$ dual $^{9}$ to the extended Clifford multiplication $\mathrm{cl}_{+}: \Lambda_{+}^{2} \mathbb{R}^{4} \otimes \mathbb{C} \rightarrow \operatorname{End} \Delta_{4}^{+}$, i.e., $q(\psi)=\mathrm{cl}_{+}^{\top}\left(\psi \otimes \psi^{\dagger}\right)$. The commutative diagram (5.26) gives rise to the following group homomorphisms of infinite-dimensional Lie groups:

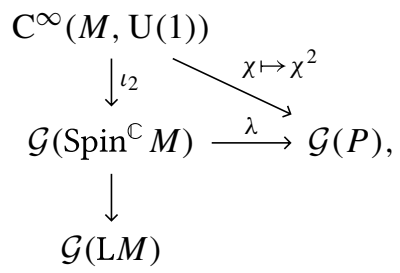

where $\mathcal{G}(P)$ is the group of gauge transformations on $P$, cf. Sect. 5.3 for its Fréchet Lie group structure. In particular, the current group $\mathcal{G}:=\mathrm{C}^{\infty}(M, \mathrm{U}(1))$ acts via gauge transformations on $\mathcal{C}(P) \times \Gamma^{\infty}\left(\Delta^{+} M\right)$ :

$$
\chi \cdot(A, \psi)=\left(A-2 \chi^{-1} \mathrm{~d} \chi, \chi \psi\right) .
$$

To formulate the Seiberg-Witten equations in the general setting discussed in the previous sections, define the map

$$
\begin{aligned}
\mathcal{S W}: \mathcal{C}(P) \times \Gamma^{\infty}\left(\Delta^{+} M\right) & \rightarrow \Omega_{+}^{2}(M, \mathrm{i} \mathbb{R}) \times \Gamma^{\infty}\left(\Delta^{-} M\right), \\
(A, \psi) & \mapsto\left(F_{A}^{+}-q(\psi), \mathrm{D}_{A} \psi\right) .
\end{aligned}
$$

A straightforward calculation (see, e.g., [58, Proposition 2.1.9]) shows that $\mathcal{S W}$ is equivariant with respect to the following $\mathcal{G}$-action on $\Omega_{+}^{2}(M, \mathrm{i} \mathbb{R}) \times \Gamma^{\infty}\left(\Delta^{-} M\right)$ :

$$
\chi \cdot(\alpha, \varphi) \mapsto(\alpha, \chi \varphi) .
$$

For $\mu \in \Omega_{+}^{2}(M, \mathrm{i} \mathbb{R})$, the Seiberg-Witten moduli space $\mathcal{M}_{\mu}$ is defined as the space of solutions $(A, \psi)$ of the Seiberg-Witten equations (5.31) modulo $\mathcal{G}$, i.e.,

\footnotetext{
${ }_{9}$ Alternatively, $q$ is the momentum map for the natural SU(2)-action on $\Delta_{4}^{+} \simeq \mathbb{C}^{2}$ under the identification $\Lambda_{+}^{2} \mathbb{R}^{4} \simeq \mathbb{R}^{3}$.
} 


$$
\mathcal{M}_{\mu}=\mathcal{S} \mathcal{W}^{-1}(\mu, 0) / \mathcal{G}
$$

Let us verify that the assumptions of Theorem 4.13 are met for the model under consideration:

1. The stabilizer of $(\mu, 0) \in \Omega_{+}^{2}(M, \mathrm{iR}) \times \Gamma^{\infty}\left(\Delta^{-} M\right)$ under the action (5.35) is the whole group $\mathcal{G}$, which is a geometric tame Fréchet Lie group with Lie algebra $\mathrm{C}^{\infty}(M, \mathrm{i} \mathbb{R})$.

2. The natural action of $\mathcal{G}(P)$ on $\mathcal{C}(P)$ is proper and admits a slice at every point as discussed in Sect. 5.3. Thus, also the action of $\mathcal{G}$ on $\mathcal{C}(P)$ admits a slice $\mathcal{S}_{A}$ at every $A \in \mathcal{C}(P)$. The stabilizer $\mathcal{G}_{A}$ of $A$ is $\mathrm{U}(1)$, viewed as constant maps. Moreover, the linear continuous action $(z, \psi) \mapsto z \psi$ of the compact group $\mathcal{G}_{A} \simeq \mathrm{U}(1)$ on the Fréchet space $\Gamma^{\infty}\left(\Delta^{+} M\right)$ admits a slice $\mathcal{S}_{\psi}$ at every $\psi \in \Gamma^{\infty}\left(\Delta^{+} M\right)$ according to [16, Theorem 3.15]. By [16, Proposition 3.29], the $\mathcal{G}$-action (5.33) on $\mathcal{C}(P) \times \Gamma^{\infty}\left(\Delta^{+} M\right)$ has a slice $\mathcal{S}_{A, \psi}=\mathcal{S}_{A} \times \mathcal{S}_{\psi}$ at $(A, \psi)$. The existence of slices in the Banach setting is well known, see, e.g., [58, Proposition 2.2.7].

3. The action (5.35) of $\mathcal{G}$ on $\Gamma^{\infty}\left(\Delta^{-} M\right) \times \Omega_{+}^{2}(M, \mathrm{i} \mathbb{R})$ is clearly linear.

4. The linearization of $\mathcal{S W}$ at a point $(A, \psi)$ is given by

$$
\begin{aligned}
& \mathrm{T}_{(A, \psi)} \mathcal{S W}: \Omega^{1}(M, \mathrm{i} \mathbb{R}) \times \Gamma^{\infty}\left(\Delta^{+} M\right) \rightarrow \Omega_{+}^{2}(M, \mathrm{i} \mathbb{R}) \times \Gamma^{\infty}\left(\Delta^{-} M\right), \\
& (\alpha, \varphi) \mapsto\left(\mathrm{d}^{+} \alpha-\dot{q}(\psi, \varphi), \mathrm{D}_{A} \varphi+\frac{1}{2} \operatorname{cl}(\alpha, \psi)\right),
\end{aligned}
$$

where $\dot{q}(\psi, \varphi)=\mathrm{cl}_{+}^{\top}\left(\psi \otimes \varphi^{\dagger}+\varphi \otimes \psi^{\dagger}\right)$ and $\mathrm{d}^{+}$denotes the self-dual part of the exterior derivative, see $\left[58\right.$, p. 125]. Moreover, the action of the Lie algebra $C^{\infty}(M, \mathbb{R})$ induced by the action (5.33) is given by

$$
\xi .(A, \psi)=(-2 \mathrm{~d} \xi, \xi \psi) \equiv \tau_{(A, \psi)}(\xi),
$$

for $\xi \in \mathrm{C}^{\infty}(M, \mathrm{i} \mathbb{R})$.

For a solution $\left(A_{0}, \psi_{0}\right) \in \mathcal{S} \mathcal{W}^{-1}(\mu, 0)$ of the Seiberg-Witten equations, the chain (4.14) here takes the form

$$
\Omega^{0}(M, \mathrm{i} \mathbb{R}) \stackrel{\tau_{(A, \psi)}}{\longrightarrow} \Omega^{1}(M, \mathrm{i} \mathbb{R}) \times \Gamma^{\infty}\left(\Delta^{+} M\right) \stackrel{\mathrm{T}_{(A, \psi)} \mathcal{S} \mathcal{W}}{\longrightarrow} \Omega_{+}^{2}(M, \mathrm{i} \mathbb{R}) \times \Gamma^{\infty}\left(\Delta^{-} M\right),
$$

where $A \in \mathcal{S}_{A_{0}}$ and $\psi \in \mathcal{S}_{\psi_{0}}$. This chain is clearly a chain of linear differential operators tamely parametrized by $(A, \psi)$. At $A=A_{0}$, the chain (5.39) is a complex that, after ignoring the zeroth order contributions (which are compact operators and thus do not change ellipticity or the index), is given by

$$
\Omega^{0}(M, \mathrm{i} \mathbb{R}) \stackrel{(\mathrm{d}, 0)}{\longrightarrow} \Omega^{1}(M, \mathrm{i} \mathbb{R}) \times \Gamma^{\infty}\left(\Delta^{+} M\right) \stackrel{\left(\mathrm{d}^{+}, \mathrm{D}_{A_{0}}\right)}{\longrightarrow} \Omega_{+}^{2}(M, \mathrm{i} \mathbb{R}) \times \Gamma^{\infty}\left(\Delta^{-} M\right) .
$$

It is known (e.g., [58, Lemma 2.2.10]) that this complex is elliptic and that its Euler characteristic is given by

$$
\frac{1}{4}\left(2 \chi_{M}+3 \sigma_{M}-\mathrm{c}_{1}(P)^{2}\right),
$$


where $\chi_{M}$ is the Euler number and $\sigma_{M}$ is the signature of $M$.

Hence, by Theorem 4.13 , the map $\mathcal{S W}$ can be brought into an equivariant normal form at every solution $(A, \psi)$ of the Seiberg-Witten equations. Moreover, as a consequence of Theorem 5.3 and Remark 5.4, we obtain the following description of the local geometry of the moduli space $\mathcal{M}_{\mu}$.

Theorem 5.9 Let $M$ be a closed oriented Riemannian manifold of dimension 4 endowed with a $\operatorname{Spin}^{\mathbb{C}}$-structure. For every $\mu \in \Omega_{+}^{2}(M, \mathrm{i} \mathbb{R})$, the Seiberg-Witten moduli space $\mathcal{M}_{\mu}$ admits a finite-dimensional Kuranishi chart at every point. Moreover, the virtual dimension of $\mathcal{M}_{\mu}$ is given by

$$
\frac{1}{4}\left(\mathrm{c}_{1}(P)^{2}-2 \chi_{M}-3 \sigma_{M}\right)
$$

Let us describe the constructed Kuranishi charts on $\mathcal{M}_{\mu}$ in more detail. For this purpose, let $(A, \psi)$ be a solution of the Seiberg-Witten equations. According to Remark 5.4 , the linear spaces occurring in the definition of a Kuranishi chart at $[A, \psi] \in \mathcal{M}_{\mu}$ are given by the finite-dimensional cohomology groups

$$
\begin{aligned}
& \mathcal{E}=\mathrm{H}_{A, \psi}^{1}(M) \equiv \operatorname{Ker}_{(A, \psi)} \mathcal{S} \mathcal{W} / \operatorname{Im} \tau_{(A, \psi)}, \\
& \mathcal{F}=\mathrm{H}_{A, \psi}^{2}(M) \equiv \Omega_{+}^{2}(M, \mathrm{iR}) \times \Gamma^{\infty}\left(\Delta^{-} M\right) / \operatorname{Im~T}_{(A, \psi)} \mathcal{S W} .
\end{aligned}
$$

The obstruction map is a $\mathcal{G}_{A, \psi}$-equivariant map

$$
\text { s: } \mathrm{H}_{A, \psi}^{1}(M) \supseteq \mathcal{V} \rightarrow \mathrm{H}_{A, \psi}^{2}(M),
$$

where $\mathcal{V}$ is a $\mathcal{G}_{A, \psi}$-invariant, open neighborhood of 0 in $\mathrm{H}_{A, \psi}^{1}(M)$. Finally, the moduli space $\mathcal{M}_{\mu}$ in a neighborhood of $[A, \psi]$ is modeled on the quotient $s^{-1}(0) / \mathcal{G}_{A, \psi}$. In this way, we recover the well-known result concerning the local structure of $\mathcal{M}_{\mu}$, see, e.g., [58, Proposition 2.2.16].

\subsection{Application: pseudoholomorphic immersions}

In this section, we discuss the moduli space of pseudoholomorphic curves. For the sake of simplicity, we consider only the simplest case of the universal Gromov-Witten moduli space without marked points.

Let $(M, \omega)$ be a finite-dimensional symplectic manifold endowed with a compatible almost complex structure $J$. For every compact Riemann surface $(\Sigma, j)$ with complex structure $j$, consider the Cauchy-Riemann operator

$$
\bar{\partial}_{j, J} u=\frac{1}{2}(\mathrm{~T} u+J \circ \mathrm{T} u \circ j),
$$

where $u: \Sigma \rightarrow M$ is a smooth map. In the following, we restrict attention to the case when $u$ is an immersion. This is to ensure the existence of slices. Denote the space of immersions of $\Sigma$ into $M$ by $\mathcal{I}(\Sigma, M)$. It is an open and dense subset of the Fréchet manifold $\mathrm{C}^{\infty}(\Sigma, M)$, see [55, Corollary 6.13]. Let $\beta \in \mathrm{H}_{2}(M, \mathbb{Z})$ be a homology class, and consider the open subset $\mathcal{I}_{\beta}(\Sigma, M)$ of $\mathcal{I}(\Sigma, M)$ consisting of immersions $u: \Sigma \rightarrow M$ with $u_{*}[\Sigma]=\beta$. Denote 
the space of complex structures on $\Sigma$ by $\mathcal{J}(\Sigma)$. As every almost complex structure on a surface is integrable, $\mathcal{J}(\Sigma)$ can be identified with the space of reductions of the frame bundle $\mathrm{L} \Sigma$ to $\mathrm{U}(1)$, i.e., $\mathcal{J}(\Sigma)=\Gamma^{\infty}\left(\mathrm{L} \Sigma \times_{\mathrm{GL}(2, \mathbb{R})} \mathrm{GL}(2, \mathbb{R}) / \mathrm{GL}(1, \mathbb{C})\right)$, and thus, it carries a natural Fréchet manifold structure. In order to realize $\bar{\partial}_{j, J}$ as a map between infinite-dimensional manifolds, let us introduce the Fréchet vector bundle $\mathcal{E} \rightarrow \mathcal{J}(\Sigma) \times \mathcal{I}(\Sigma, M)$ whose fiber over $(j, u) \in \mathcal{J}(\Sigma) \times \mathcal{I}(\Sigma, M)$ is the space $\Omega^{0,1}\left(\Sigma, u^{*} \mathrm{~T} M\right)$ of smooth $(j, J)$-antilinear 1 -forms on $\Sigma$ with values in $u^{*} \mathrm{~T} M$. The Cauchy-Riemann operator yields a smooth section

$$
\mathcal{C R}: \mathcal{J}(\Sigma) \times \mathcal{I}_{\beta}(\Sigma, M) \rightarrow \mathcal{E}, \quad(j, u) \mapsto \bar{\partial}_{j, J} u .
$$

Note that $\mathcal{C R}$ is equivariant with respect to the natural reparametrization actions of the Fréchet Lie group $\mathcal{D}(\Sigma)$ of diffeomorphisms of $\Sigma$. The associated moduli space

$$
\mathcal{M}_{\beta, J}=\mathcal{C} \mathcal{R}^{-1}(0) / \mathcal{D}(\Sigma)
$$

is the universal moduli space of unparametrized pseudoholomorphic immersions representing $\beta \in \mathrm{H}_{2}(M, \mathbb{Z})$. Here, 0 denotes the zero section in $\mathcal{E}$.

Let us verify that the assumptions of Theorem 4.13 are met for the model under consideration. To be precise, a slight variation of Theorem 4.13 is necessary as $\mathcal{M}_{\beta, J}$ is defined via the preimage of a submanifold instead of a single point. We thus have to establish the existence of a normal form relative to the submanifold as in Theorem 3.15. Since we are interested in the preimage of the zero section in $\mathcal{E}$, this simply amounts to replacing the derivative of the section in (4.19) by its vertical derivative.

1. The zero section of $\mathcal{E}$ is invariant under the action of $\mathcal{D}(\Sigma)$, which is a geometric tame Fréchet Lie group with Lie algebra $\mathfrak{X}(\Sigma)$.

2. The reparametrization action of $\mathcal{D}(\Sigma)$ on $\mathcal{I}(\Sigma, M)$ admits a slice $\mathcal{S}_{u}$ at every immersion $u \in \mathcal{I}(\Sigma, M)$, see [7]. According to [7, Lemma 3.1], the stabilizer $\mathcal{D}_{u}(\Sigma)$ of $u$ is a finite subgroup of $\mathcal{D}(\Sigma)$. Since every invariant open set is a slice for the action of a finite group, the induced action of $\mathcal{D}_{u}(\Sigma)$ on $\mathcal{J}(\Sigma)$ admits a slice $\mathcal{S}_{j}$ at every $j \in \mathcal{J}(\Sigma)$. By [16, Proposition 3.29], the $\mathcal{D}(\Sigma)$-action on $\mathcal{J}(\Sigma) \times \mathcal{I}(\Sigma, M)$ has a slice $\mathcal{S}_{j, u}=\mathcal{S}_{j} \times \mathcal{S}_{u}$ at $(j, u)$.

3. The action of $\mathcal{D}(\Sigma)$ on $\mathcal{E}$ is fiberwise linear.

4. The tangent space of $\mathcal{J}(\Sigma)$ at $j$ is the space of sections of the bundle $\operatorname{End}_{j}(\mathrm{~T} \Sigma)$ whose fiber at $x \in \Sigma$ is the space of linear maps $\xi: \mathrm{T}_{x} \Sigma \rightarrow \mathrm{T}_{x} \Sigma$ satisfying $j \circ \xi+\xi \circ j=0$. The vertical tangent map of $\mathcal{C} \mathcal{R}$ at a point $(j, u) \in \mathcal{C} \mathcal{R}^{-1}(0)$ is given by

$$
\begin{gathered}
\mathrm{V}_{(j, u)} \mathcal{C R}: \operatorname{End}_{j}(\mathrm{~T} \Sigma) \times \Gamma^{\infty}\left(u^{*} \mathrm{~T} M\right) \rightarrow \Omega^{0,1}\left(\Sigma, u^{*} \mathrm{~T} M\right), \\
(\xi, X) \mapsto\left(\mathrm{D}_{u} \xi+\frac{1}{2} J \circ \mathrm{T} u \circ \xi\right),
\end{gathered}
$$

where $\mathrm{D}_{u}: \Gamma^{\infty}\left(u^{*} \mathrm{~T} M\right) \rightarrow \Omega^{0,1}\left(\Sigma, u^{*} \mathrm{~T} M\right)$ is the usual linearized Cauchy-Riemann operator, cf. [52, Proposition 3.1.1]. As discussed above, we have to use the vertical derivative in place of the normal derivative in the chain (4.14). It here takes the form

$$
\mathfrak{X}(\Sigma) \stackrel{(\mathfrak{L} l,-\mathrm{T} v)}{\longrightarrow} \operatorname{End}_{j}(\mathrm{~T} \Sigma) \times \Gamma^{\infty}\left(u^{*} \mathrm{~T} M\right) \stackrel{\mathrm{V}_{(l, v)} \mathcal{C R}}{\longrightarrow} \Omega^{0,1}\left(\Sigma, u^{*} \mathrm{~T} M\right),
$$

where $l \in \mathcal{S}_{j}$ and $v \in \mathcal{S}_{u}$. This chain is a chain of linear differential operators tamely parametrized by $(l, v)$. At $(l, v)=(j, u)$, the chain (5.49) is a complex that, after 
ignoring the zeroth-order contributions (which are compact operators and thus do not change ellipticity or the index), is given by

$$
\mathfrak{X}(\Sigma) \stackrel{(\mathfrak{L} j, 0)}{\longrightarrow} \operatorname{End}_{j}(\mathrm{~T} \Sigma) \times \Gamma^{\infty}\left(u^{*} \mathrm{~T} M\right) \stackrel{\mathrm{D}_{u}}{\longrightarrow} \Omega^{0,1}\left(\Sigma, u^{*} \mathrm{~T} M\right) .
$$

This complex is elliptic because $\mathfrak{2} j: \mathfrak{X}(\Sigma) \rightarrow \operatorname{End}_{j}(\mathrm{~T} \Sigma)$ is elliptic with index (cf. [59], Proposition 9.4.4; [52], Theorem C.1.10])

$$
\text { ind } \mathfrak{R} j=(2-2 g)+2\left\langle\mathrm{c}_{1}\left(\mathrm{~T}^{1,0} \Sigma\right),[\Sigma]\right\rangle=3(2-2 g)
$$

and $\mathrm{D}_{u}: \Gamma^{\infty}\left(u^{*} \mathrm{~T} M\right) \rightarrow \Omega^{0,1}\left(\Sigma, u^{*} \mathrm{~T} M\right)$ is elliptic with index (cf. [52, Theorem C.1.10])

$$
\text { ind } \mathrm{D}_{u}=n(2-2 g)+2\left\langle\mathrm{c}_{1}\left(u^{*} \mathrm{~T} M\right),[\Sigma]\right\rangle \text {, }
$$

where $2 n=\operatorname{dim}_{\mathbb{R}} M$. Thus, the Euler characteristic of (5.50) is given by

$$
\text { ind } \Omega_{j}-\text { ind } \mathrm{D}_{u}=(3-n)(2-2 g)-2\left\langle\mathrm{c}_{1}\left(u^{*} \mathrm{~T} M\right),[\Sigma]\right\rangle .
$$

Hence, by Theorem 4.13 and the tame Fréchet version of Theorem 3.15, the map $\mathcal{C} \mathcal{R}$ can be brought into an equivariant normal form relative to the zero section at every pseudoholomorphic curve $(j, u)$. Moreover, as a consequence of Theorem 5.3 and Remark 5.4, we obtain the following description of the local geometry of the moduli space $\mathcal{M}_{\beta, J}$.

Theorem 5.10 Let $\Sigma$ be a closed, oriented surface and let $(M, \omega, J)$ be a symplectic manifold of dimension $2 n$ endowed with a compatible almost complex structure J. For every $\beta \in \mathrm{H}_{2}(M, \mathbb{Z})$, the moduli space $\mathcal{M}_{\beta, J}$ of unparametrized pseudoholomorphic immersions admits a finite-dimensional Kuranishi chart at every point $[j, u] \in \mathcal{M}_{\beta, J}$. Moreover, the virtual dimension of $\mathcal{M}_{\beta, J}$ is given by

$$
(n-3)(2-2 g)+2\left\langle\mathrm{c}_{1}(M), \beta\right\rangle .
$$

In the study of pseudoholomorphic curves, one is usually not only interested in immersions but also in more general smooth or nodal curves. We have restricted attention to the open subset $\mathcal{I}(\Sigma, M) \subseteq \mathrm{C}^{\infty}(\Sigma, M)$ of immersions to ensure that the reparametrization action of $\mathcal{D}(\Sigma)$ admits slices. The discussion in [7] shows that, in general, one cannot expect the existence of slices for the reparametrization action on the space $\mathrm{C}^{\infty}(\Sigma, M)$ of all smooth maps. In particular, the slice property $\mathrm{SL}_{2}$ is problematic and it is not even clear whether the quotient $\mathrm{C}^{\infty}(\Sigma, M) / \mathcal{D}(\Sigma)$ be Hausdorff. However, as we have discussed in Remark 5.5, for the Kuranishi structure of the moduli space one only needs $\mathrm{SL}_{2}$ to hold for solutions $(j, u)$ of the Cauchy-Riemann equation $\mathcal{C R}(j, u)=0$. Such a weaker version of $\mathrm{SL}_{2}$ can be established using Gromov's compactness theorem as in [27], p. 999; [26], Lemma 20.15]. Using this observation, one can then also construct Kuranishi charts on the moduli space of unparametrized pseudoholomorphic (stable) curves.

The existence of Kuranishi charts on $\mathcal{M}_{\beta, J}$ is well known, see, e.g., [27, Theorem 12.9] and references therein. However, the traditional approach using Banach spaces faces serious technical problems due to the fact that the reparametrization action is not smooth with respect to maps of a given Sobolev class, see, for example, [54, Section 3] for a detailed discussion. These difficult issues have led to considerable discussion about the correctness of the foundations of the theory of pseudoholomorphic curves, see [25, 26, 53, 54] and 
references therein. Moreover, these issues inspired the development of the so-called scale calculus that provides the analytic backbone of the polyfold framework [40]. Our construction using the Nash-Moser theorem has the important advantage that we circumvent such technical problems completely, since the reparametrization action of the diffeomorphism group on the space of smooth maps is a tame smooth action. Thus, phrasing the problem in the well-established framework of tame Fréchet manifolds allows us to concentrate on the geometric constructions rather than the analytic details.

\section{Outlook}

In this paper, local normal form theorems for smooth equivariant maps between infinitedimensional manifolds are established in various analytic settings. As we have seen, these equivariant normal form theorems are a powerful tool to study the local structure of moduli spaces and to show that these moduli spaces carry the structure of a Kuranishi space, i.e., they are locally modeled on the quotient by a compact group of the zero set of a smooth map. Using this general framework, we were able to give short and unified proofs that the moduli space of anti-self-dual instantons, the Seiberg-Witten moduli space and the moduli space of pseudoholomorphic curves admit Kuranishi charts.

The normal forms developed in this paper are flexible enough to respect further geometric data. In forthcoming work [14] (see also [13]), we refine and adapt the techniques developed in this paper to Hamiltonian actions on infinite-dimensional symplectic manifolds. We construct a normal form for equivariant momentum maps in the spirit of the classical Marle-Guillemin—Sternberg normal form. The fundamental idea is to use Theorem 4.6 to bring the momentum map into a normal form and then gain control over the behavior of the singular part of the momentum map using the symplectic form. With the help of this normal form, we then prove a singular symplectic reduction theorem including the analysis of the stratification into symplectic manifolds. This strategy is completely different to the traditional proof of the finite-dimensional Marle-Guillemin-Sternberg theorem which uses an equivariant version of Darboux's theorem. A simple counterexample by Marsden [50] shows that the Darboux theorem fails spectacularly already for weakly symplectic Banach manifolds, so this approach is not possible in infinite dimensions. In physics, one is mainly interested in the case where the symplectic manifold is a cotangent bundle. A normal form of the momentum map for a lifted action on an infinite-dimensional cotangent bundle is established in [17].

Our results concerning equivariant normal forms in infinite dimensions and the techniques developed in the previous chapters open many exciting avenues for further research. We list some relevant open problems and fundamental issues:

1. It would be very interesting to extend the discussion of the normal form of a smooth map to higher orders. One would expect that the knowledge of higher-order terms of the Taylor expansion of a smooth map $f$ yields further control over the behavior of its singular part $f_{\text {sing }}$. In this way, one would gain deeper insight into the singular structure of the level sets of $f$. This also opens the path toward an infinite-dimensional Morse theory.

2. Studying the local structure of moduli spaces is the first step in an elaborated program to define powerful geometric invariants. Usually, one then passes to a compactification and constructs a virtual fundamental cycle for the compactified moduli space. Thus, it is 
desirable to have a general scheme in the Fréchet framework for the issues of compactification and perturbation, similarly to the aim of the polyfold theory of Hofer, Wysocki, and Zehnder [40].

3. In Sect. 5, we studied moduli spaces for which the deformation complex is elliptic. The Nash-Moser techniques also allow to study problems that are not accessible to elliptic methods. Thus, it would be interesting to apply our general framework to, say, KAM theory, to deformations of fibrations or to normal forms in Poisson geometry.

\section{Appendix A: Inverse function theorems}

In this section, we give a brief overview of different generalizations of the classical inverse function theorem to the infinite-dimensional setting. The primary focus is on Glöckner's inverse function theorem for maps between Banach spaces with parameters in a locally convex space and on the Nash-Moser theorem in the tame Fréchet category.

As a reference point, let us recall the classical version of the inverse function theorem in the Banach setting.

Theorem A.1 Banach version, [47, Theorem I.5.2] Let X, Y be Banach spaces and let $f: X \supseteq U \rightarrow Y$ be a smooth map defined on an open neighborhood $U$ of 0 in $X$. If $\mathrm{T}_{0} f: X \rightarrow Y$ is an isomorphism of Banach spaces, then $f$ is a local diffeomorphism at 0 .

$[29,30]$ has established the following generalization of the Banach inverse function theorem to smooth maps depending on parameters in a locally convex space. Similar results have been obtained in [39] (using a slightly stronger notion of differentiability) and in [80] (using the so-called convenient calculus).

Theorem A.2 (Banach version with parameters, [30, Theorem 2.3]) Let $P \subseteq E$ be an open neighborhood of 0 in the locally convex space E. Let $X, Y$ be Banach spaces, let $U$ be an open neighborhood of 0 in $X$ and let $f: E \times X \supseteq P \times U \rightarrow Y$ be a smooth map. If the partial derivative $\mathrm{T}_{(0,0)}^{2} f: X \rightarrow Y$ of $f$ at $(0,0)$ with respect to the second variable is an isomorphism of Banach spaces, then the map

$$
E \times X \supseteq P \times U \rightarrow E \times Y, \quad(p, x) \mapsto(p, f(p, x))
$$

is a local diffeomorphism at $(0,0)$.

We now recall the main notions of the tame Fréchet category and the Nash-Moser inverse function theorem, cf. [35]. A Fréchet space $X$ is called graded if it carries a distinguished increasing fundamental system of seminorms $\|\cdot\|_{k}$. A graded Fréchet space is called tame if the seminorms satisfy an additional interpolation property, which formalizes the idea that $X$ admits smoothing operators, see [35, Definition II.1.3.2] for the exact statement. Let $X$ and $Y$ be tame Fréchet spaces. A continuous (possibly nonlinear) map $f: X \supseteq U \rightarrow Y$ defined on an open subset $U \subseteq X$ is $r$-tame if it satisfies a local estimate of the form

$$
\|f(x)\|_{k} \leq C\left(1+\|x\|_{k+r}\right)
$$


Roughly speaking, this means that $f$ has a maximal loss of $r$ derivatives. Moreover, a smooth map $f$ is called $r$-tame smooth if $f$ and all its derivatives $\mathrm{d}^{(j)} f: U \times X^{j} \rightarrow Y$ are $r$-tame. Let $Z$ be a tame Fréchet space and assume that $Z$ is the topological direct sum of closed subspaces $X$ and $Y$. We say that the sum $Z=X \oplus Y$ is tame if the map $X \times Y \rightarrow Z$ given by $(x, y) \mapsto x+y$ is a tame isomorphism.

Theorem A.3 (Nash-Moser inverse function theorem, [35, Section III.1]) Let $X$ and $Y$ be tame Fréchet spaces, let $U$ be an open neighborhood of 0 in $X$ and let $f: X \supseteq U \rightarrow Y$ be a tame smooth map. Assume that the derivative $\mathrm{T} f$ has a tame smooth family $\psi$ of inverses, that is, $\psi: U \times Y \rightarrow X$ is a tame smooth map and $\psi(x, \cdot): Y \rightarrow X$ is inverse to $\mathrm{T}_{x} f$ for every $x \in U$. Then the map $f$ is a tame local diffeomorphism at 0 .

The important point is that the derivative of $f$ has to be invertible in a neighborhood of 0 and that one requires tame estimates for the inverses.

\section{B Slices and orbit-type stratification}

In this section, we give a brief account of the theory of Lie group actions in the category of locally convex manifolds. The focus lies on slices for the action and the stratification of the manifold into orbit types. We refer the reader to [16] for more details.

Let $M$ be a (locally convex) manifold. Assume a (locally convex) Lie group $G$ acts smoothly on $M$, that is, assume that the action map $G \times M \rightarrow M$ is smooth. We refer to this setting by saying that $M$ is a $G$-manifold. The action is often written, using the dot notation, as $(g, m) \mapsto g \cdot m$. Similarly, the induced action of the Lie algebra $\mathfrak{g}$ of $G$ is denoted by $\xi . m \in \mathrm{T}_{m} M$ for $\xi \in \mathfrak{g}$ and $m \in M$. Clearly, $m \mapsto \xi . m$ is the Killing vector field generated by $\xi$. Furthermore, $G \cdot m=\{g \cdot m: g \in G\} \subseteq M$ is the orbit through $m \in M$. The $G$-action is called proper if inverse images of compact subsets under the map

$$
G \times M \rightarrow M \times M, \quad(g, m) \mapsto(g \cdot m, m)
$$

are compact.

The subgroup $G_{m}:=\{g \in G: g \cdot m=m\}$ is called the stabilizer subgroup of $m \in M$. It is not known, even for Banach Lie group actions, whether $G_{m}$ is always a Lie subgroup, see [57, Problem IX.3.b]. However, for proper actions this is the case, see [16, Lemma 2.11]. In fact, then $G_{m}$ is even a principal Lie subgroup of $G$, which means that the natural fibration $G \rightarrow G / G_{m}$ is a principal bundle. The $G$-action is called free if all stabilizer subgroups are trivial. Two subgroups $H$ and $K$ of $G$ are said to be conjugate if there exists $a \in G$ such that $a H a^{-1}=K$; we write $H \sim K$ is this case. In view of the equivariance relation $G_{g \cdot m}=g G_{m} g^{-1}$, for every $m \in M$ and $g \in G$, we can assign to every orbit $G \cdot m$ the conjugacy class $\left(G_{m}\right)$, which is called the orbit type of $m$. We put a preorder on the set of orbit types by declaring $(H) \leq(K)$ for two orbit types, represented by the stabilizer subgroups $H$ and $K$, if there exists $a \in G$ such that $a H a^{-1} \subseteq K$. If the action is proper, this preorder is actually a partial order. For every closed subgroup $H \subseteq G$, define the following subsets of $M$ :

$$
\begin{aligned}
M_{H} & =\left\{m \in M: G_{m}=H\right\}, \\
M_{(H)} & =\left\{m \in M:\left(G_{m}\right)=(H)\right\} .
\end{aligned}
$$


The subset $M_{H}$ is called the isotropy type subset and $M_{(H)}$ is the subset of orbit type $(H)$. Analogous definitions hold for every subset $N \subseteq M$, so, for example, $N_{H}=N \cap M_{H}$.

As in finite dimensions, the local structure of the orbit-type subsets is studied with the help of slices, cf. Proposition B.3. Slices also play a fundamental role in the construction of the normal form of an equivariant map in Sect. 4.

Definition B. 1 Let $M$ be a $G$-manifold. A slice at $m \in M$ is a submanifold $S \subseteq M$ containing $m$ with the following properties:

$\mathrm{SL}_{1} \quad$ The submanifold $S$ is invariant under the induced action of the stabilizer subgroup $G_{m}$, that is $G_{m} \cdot S \subseteq S$.

$\mathrm{SL}_{2} \quad$ Any $g \in G$ with $(g \cdot S) \cap S \neq \emptyset$ is necessarily an element of $G_{m}$.

$\mathrm{SL}_{3}$ The stabilizer $G_{m}$ is a principal Lie subgroup of $G$ and the principal bundle $G \rightarrow G / G_{m}$ admits a local section $\chi: G / G_{m} \supseteq W \rightarrow G$ defined on an open neighborhood $W$ of the identity coset $[e]$ in such a way that the map

$$
\chi^{S}: W \times S \rightarrow M, \quad([g], s) \mapsto \chi([g]) \cdot s
$$

is a diffeomorphism onto an open neighborhood of $m$, which is called a slice neighborhood of $m$.

$\mathrm{SL}_{4}$ The partial slice $S_{\left(G_{m}\right)}=\left\{s \in S: G_{s}\right.$ is conjugate to $\left.G_{m}\right\}$ is a closed submanifold of $S$.

$\mathrm{SL}_{5}$ There exist a smooth representation of $G_{m}$ on a locally convex space $X$ and a $G_{m}$ -equivariant diffeomorphism $\imath_{S}$ from a $G_{m}$-invariant open neighborhood of 0 in $X$ onto $S$ such that $l_{S}(0)=m$.

The notion of a slice is closely related to the concept of a tubular neighborhood.

Proposition B.2 ([16, Proposition 2.6.2]) Let $M$ be a G-manifold. For every slice $S$ at $m \in M$, the tube map

$$
\chi^{\mathrm{T}}: G \times_{G_{m}} S \rightarrow M, \quad[g, s] \mapsto g \cdot s
$$

is a G-equivariant diffeomorphism onto an open, G-invariant neighborhood of $G \cdot \operatorname{m}$ in $M$.

In the finite-dimensional context, the existence of slices for proper actions is ensured by Palais' slice theorem [62]. Passing to the infinite-dimensional case, this may no longer be true and additional hypotheses have to be made. We refer the reader to [16, $78]$ for general slice theorems in infinite dimensions and to $[2,7,21]$ for constructions of slices for concrete examples.

As in the finite-dimensional case, the existence of slices implies many nice properties of the orbit space. For example, we have the following.

Proposition B.3 ([16, Propositions 4.1 and 4.5]) Let $M$ be a $G$-manifold with proper $G$ action. If the $G$-action admits a slice at every point of $M$, then $M_{(H)}$ is a submanifold of $M$. Moreover, $\check{M}_{(H)}=M_{(H)} / G$ carries a smooth manifold structure such that the natural projection $\pi_{(H)}: M_{(H)} \rightarrow M_{(H)}$ is a smooth submersion. 
If, in addition, a certain approximation property is satisfied, then the orbit-type manifolds fit together nicely and the orbit space is a stratified space, see [16, Theorem 4.2]. More generally, we have the following stratification result for subsets of $M$.

Proposition B.4 ([16, Proposition 4.7]) Let $M$ be a G-manifold with proper G-action and let $P$ be a closed $G$-invariant subset of $M$. Assume that the $G$-action on $M$ admits a slice $S$ at every point $m \in P$ such that the following holds:

1. $P \cap S_{\left(G_{m}\right)}$ is a closed submanifold of $S_{\left(G_{m}\right)}$.

2. For every orbit type $(K) \leq\left(G_{m}\right)$, the point $m$ lies in the closure of $P \cap S_{(K)}$ in $S$.

Then, the induced partition of $P$ into the orbit-type subsets $P_{(H)}=P \cap M_{(H)}$ is a stratification. Moreover, under these assumptions, the decomposition of $\breve{P}=P / G$ into $\check{P}_{(H)}=P_{(H)} / G$ is a stratification, too.

For completeness, we include our definition of a stratification here and refer the reader to [16] for further details and comparison with other notions of stratified spaces in the literature.

Definition B.5 Let $X$ be Hausdorff topological space. A partition $\mathcal{Z}$ of $X$ into subsets $X_{\sigma}$ indexed by $\sigma \in \Sigma$ is called a stratification of $X$ if the following conditions are satisfied:

$\left(D S_{1}\right)$ Every piece $X_{\sigma}$ is a locally closed, smooth manifold (whose manifold topology coincides with the relative topology). We will call $X_{\sigma}$ a stratum of $X$.

$\left(D S_{2}\right) \quad$ (frontier condition) Every pair of disjoint strata $X_{\varsigma}$ and $X_{\sigma}$ with $X_{\varsigma} \cap \overline{X_{\sigma}} \neq \emptyset$ satisfies:

(a) $X_{\varsigma}$ is contained in the frontier $\overline{X_{\sigma}} \backslash X_{\sigma}$ of $X_{\sigma}$,

(b) $X_{\sigma}$ does not intersect $\overline{X_{\varsigma}}$.

Acknowledgements T. Diez was supported by the NWO grant 639.032.734 "Cohomology and representation theory of infinite-dimensional Lie groups." We gratefully acknowledge support of the Max Planck Institute for Mathematics in the Sciences in Leipzig and of the University of Leipzig.

Open Access This article is licensed under a Creative Commons Attribution 4.0 International License, which permits use, sharing, adaptation, distribution and reproduction in any medium or format, as long as you give appropriate credit to the original author(s) and the source, provide a link to the Creative Commons licence, and indicate if changes were made. The images or other third party material in this article are included in the article's Creative Commons licence, unless indicated otherwise in a credit line to the material. If material is not included in the article's Creative Commons licence and your intended use is not permitted by statutory regulation or exceeds the permitted use, you will need to obtain permission directly from the copyright holder. To view a copy of this licence, visit http://creativecommons.org/licenses/by/4.0/.

\section{References}

1. Abbati, M.C., Cirelli, R., Manià, A., Michor, P.: Smoothness of the action of the gauge transformation group on connections. J. Math. Phys. 27(10), 2469-2474 (1986)

2. Abbati, M.C., Cirelli, R., Manià, A.: The orbit space of the action of gauge transformation group on connections. J. Geom. Phys. 6(4), 537-557 (1989) 
3. Atiyah, M.F., Bott, R.: A Lefschetz fixed point formula for elliptic complexes, I. Ann. Math. 86(2), 374-407 (1967)

4. Atiyah, M.F., Bott, R.: The Yang-Mills equations over Riemann surfaces. Philos. Trans. R. Soc. Lond. Ser. A. 308(1505), 523-615 (1993)

5. Atiyah, M.F., Hitchin, N.J., Singer, I.M.: Self-duality in four-dimensional Riemannian geometry. Proc. R. Soc. Lond. Ser. A 362(1711), 425-461 (1978)

6. Bochner, S.: Compact groups of differentiable transformations. Ann. Math. 46(3), 372-381 (1945)

7. Cervera, V., Mascaró, F., Michor, P.W.: The action of the diffeomorphism group on the space of immersions. Differ. Geom. Appl. 1(4), 391-401 (1991)

8. Chang, K.C.: Methods in Nonlinear Analysis: Springer Monographs in Mathematics. Springer, Berlin (2005)

9. Chossat, P., Lewis, D., Ortega, J.-P., Ratiu, T.S.: Bifurcation of relative equilibria in mechanical systems with symmetry. Adv. Appl. Math. 31(1), 10-45 (2003). arXiv:math/9912232

10. Cirelli, R., Manià, A.: The group of gauge transformations as a Schwartz-Lie group. J. Math. Phys. 26(12), 3036-3041 (1985)

11. Conn, J.F.: Normal forms for smooth poisson structures. Ann. Math. 121(3), 565 (1985)

12. Diez, T.: Slice Theorem for Fréchet Group Actions and Covariant Symplectic Field Theory. MA Thesis. Universität Leipzig (2013). arXiv:1405.2249

13. Diez, T.: Normal form of equivariant maps and singular symplectic reduction in infinite dimensions with applications to gauge field theory. PhD thesis. Universität Leipzig (2019). arXiv:1909.00744 [math.SG]

14. Diez, T.: Singular symplectic reduction in infinite dimensions (in preparation)

15. Diez, T., Huebschmann, J.: Yang-Mills moduli spaces over an orientable closed surface via Fréchet reduction. J. Geom. Phys. 132, 393-414 (2018). arXiv: 1704.01982

16. Diez, T., Rudolph, G.: Slice theorem and orbit type stratification in infinite dimensions. Differential. Geom. Appl. 65, 176-211 (2019). arXiv:1812.04698 [math.DG]

17. Diez, T., Rudolph, G.: Singular symplectic cotangent bundle reduction of gauge field theory. J. Math. Phys. 61, 092902 (2020). arXiv: 1812.04707 [math-ph]

18. Donaldson, S.K.: An application of gauge theory to four-dimensional topology. J. Differential Geom. 18(2) (1983), 279-315

19. Donaldson, S.K., Kronheimer, P.B.: The Geometry of Four-Manifolds. Oxford University Press, Oxford Mathematical Monographs (1997)

20. Duistermaat, J.J., Kolk, J.A.C.: Lie Groups. Springer, Berlin Heidelberg (1999)

21. Ebin, D.G.: The manifold of Riemannian metrics. In: Proceedings of Symposia in Pure Mathematics, vol. 15, American Mathematical Society, pp. 11-40 (1970)

22. Edwards, R.E.: Functional Analysis. Theory and applications. Holt, Rinehart and Winston, New YorkToronto-London (1965)

23. Eliashberg, Y., Givental, A., Hofer, H.: Introduction to Symplectic Field Theory (2000). arXiv:math/ 0010059

24. Freed, D.S., Uhlenbeck, K.K.: Instantons and Four-Manifolds. Mathematical Sciences Research Institute Publications, Springer (1984)

25. Fukaya, K., Oh, Y.-G., Ohta, H., On, K.: Construction of Kuranishi Structures on the Moduli Spaces of Pseudo Holomorphic Disks: I (2017). arXiv:1710.01459 [math]

26. Fukaya, K., Oh, Y.-G., Ohta, H., Ono, K.: Technical details on Kuranishi structure and virtual fundamental chain (2012). arXiv:1209.4410 [math]

27. Fukaya, K., Ono, K.: arnold conjecture and Gromov-Witten invariant. Topology 38(5), 933-1048 (1999)

28. Gerstenberger, A.: A version of scale calculus and the associated Fredholm theory (2016). arXiv:1602. 07108 [math]

29. Glöckner, H.: Finite order differentiability properties, fixed points and implicit functions over valued fields (2005). arXiv:math/0511218

30. Glöckner, H.: Implicit functions from topological vector spaces to Banach spaces. Israel J. Math. 155, 205-252 (2006)

31. Glöckner, H.: Fundamentals of submersions and immersions between infinite-dimensional manifolds (2015). arXiv: 1502.05795 [math.DG]

32. Gromov, M.: Pseudo holomorphic curves in symplectic manifolds. Invent. Math. 82(2), 307-347 (1985)

33. Guillemin, V., Lerman, E., Sternberg, S.: Symplectic Fibrations and Multiplicity Diagrams. Cambridge University Press (1996)

34. Hamilton, R.S.: Deformation theory of foliations. Preprint available from Cornell University (1978) 
35. Hamilton, R.S.: The inverse function theorem of Nash and Moser. Bull. Amer. Math. Soc 7, 65-223 (1982)

36. Harte, R.: Invertibility And Singularity For Bounded Linear Operators. CRC Press (1987)

37. Hertsch, A., Rudolph, G., Schmidt, M.: On the gauge orbit types for theories with classical compact gauge group. Rep. Math. Phys. 66(3), 331-353 (2010)

38. Hertsch, A., Rudolph, G., Schmidt, M.: Gauge Orbit Types for Theories with Gauge Group O(n), SO(n) or Sp(n). Ann. Henri Poincaré 12(2), 351-395 (2011). arXiv:0812.0228

39. Hiltunen, S.: Implicit functions from locally convex spaces to Banach spaces. Studia Math. 134(3), 235-250 (1999)

40. Hofer, H., Wysocki, K., Zehnder, E.: Polyfold and Fredholm Theory (2017). arXiv:1707.08941 [math]

41. Hofer, H.H., Wysocki, K., Zehnder, E.: Polyfold and fredholm theory I: basic theory in M-polyfolds. Mem. Amer. Math. Soc. 248(1179) (2017). arXiv:1407.3185 [math]

42. Hörmander, L.: The Analysis of Linear Partial Differential Operators III. Springer, Pseudo-Differential Operators (2007)

43. Joyce, D.: A new definition of Kuranishi space (2014). arXiv:1409.6908v3 [math.DG]

44. Kondracki, W., Rogulski, J.: On the stratification of the orbit space for the action of automorphisms on connections. Dissertationes Math. 250 (1986)

45. Köthe, G.: Topological Vector Spaces I. Springer, Berlin Heidelberg (1983)

46. Kuranishi, M.: New proof for the existence of locally complete families of complex structures. In: Aeppli, A., Calabi, E., Röhrl, H (eds.), Proceedings of the Conference on Complex Analysis, Springer, Berlin, pp. 142-154 (1965)

47. Lang, S.: Fundamentals of Differential Geometry. Graduate Texts in Mathematics, vol. 191, Springer, New York (1999)

48. Marcut, I.: Rigidity around Poisson submanifolds. Acta Mathematica 213(1), 137-198 (2014)

49. Margalef-Roig, J., Dominguez, E.O.: Differential Topology. North Holland, North-Holland Mathematics Studies (1992)

50. Marsden, J.: Darboux's Theorem Fails for Weak Symplectic Forms. Proc. Amer. Math. Soc. 32(2), 590-592 (1972)

51. Marsden, J.E., Ratiu, T., Abraham, R.: Manifolds, Tensor Analysis, and Applications, 3rd edn. Applied Mathematical Sciences. Springer, New York (2002)

52. McDuff, D., Salamon, D.: J-Holomorphic Curves and Symplectic Topology, 2nd edn. American Mathematical Society, 726 pp (2012)

53. McDuff, D., Wehrheim, K.: Kuranishi atlases with trivial isotropy-the 2013 state of affairs (2015). arXiv:1208.1340 [math]

54. McDuff, D., Wehrheim, K.: The fundamental class of smooth Kuranishi atlases with trivial isotropy. J. Topol. Anal. 10(01), 71-243 (2018). arXiv:1508.01560 [math]

55. Michor, P.W.: Manifolds of Differentiable Mappings. Birkhauser (1980)

56. Moore, E.H.: On the Reciprocal of the General Algebraic Matrix. Bull. Amer. Math. Soc. 26, 394-395 (1920)

57. Neeb, K.-H.: Towards a Lie theory of locally convex groups. Japan. J. Math. 1(2), 291-468 (2006)

58. Nicolaescu, L.: Notes on Seiberg-Witten theory. American Mathematical Society, Providence, R.I (2000)

59. Oh, Y.-G.: Symplectic Topology and Floer Homology, Cambridge University Press, Cambridge, 420 pp (2015)

60. Oh, Y.-G., Ohta, H., Ono, K., Fukaya, K.: Lagrangian Intersection Floer Theory: Anomaly and Obstruction, Part II. Studies in Advanced Mathematics, American Mathematical Society (2009)

61. Ortega, J.P., Ratiu, T.S.: Momentum Maps and Hamiltonian Reduction, Birkhäuser, Boston (2004)

62. Palais, R.S.: On the Existence of Slices for Actions of Non-Compact Lie Groups. Ann. of Math. 73(2), 295-323 (1961)

63. Palais, R.S.: Foundations of global non-linear analysis. Benjamin, New York (1968)

64. Penrose, R.: A generalized inverse for matrices. Math. Proc. Cambridge Philos. Soc. 51(03), 406-413 (1955)

65. Pflaum, M.J., Wilkin, G.: Equivariant control data and neighborhood deformation retractions (2017). arXiv: 1706.09539

66. Rao, C.R.: A Note on a Generalized Inverse of a Matrix with Applications to Problems in Mathematical Statistics. J. Roy. Statist. Soc. Ser. B 24(1), 152-158 (1962)

67. Rudolph, G., Schmidt, M., Volobuev, I.P.: Classification of gauge orbit types for SU(n)-gauge theories. Math. Phys. Anal. Geom. 5(3), 201-241 (2002). arXiv:0003044 [math-ph]

68. Rudolph, G., Schmidt, M., Volobuev, I.P.: On the gauge orbit space stratification: a review. J. Phys. A: Math. Gen. 35(28), R1-R50 (2002). arXiv:0203027 [hep-th] 
69. Rudolph, G., Schmidt, M., Volobuev, I.P.: Partial ordering of gauge orbit types for SUn-gauge theories. J. Geom. Phys. 42.1-42.2, 106-138 (2002). arXiv:0009018 [math-ph]

70. Rudolph, G., Schmidt, M: Differential Geometry and Mathematical Physics, Part II: Fibre Bundles, Topology and Gauge Fields, Springer (2017)

71. Schaefer, H.H.: Über singuläre Integralgleichungen und eine Klasse von Homomorphismen in lokalkonvexen Räumen. Math. Z. 66(1), 147-163 (1956)

72. Schaefer, H.H.: On the Fredholm alternative in locally convex linear spaces. Studia Math. 18(3), 229245 (1959)

73. Seiberg, N., Witten, E.: Electric-magnetic duality, monopole condensation, and confinement in $\mathrm{N}=2$ supersymmetric Yang-Mills theory. Nuclear Physics B 426(1), 19-52 (1994)

74. Seiberg, N., Witten, E.: Monopoles, duality and chiral symmetry breaking in $\mathrm{N}=2$ supersymmetric QCD. Nuclear Physics B 431(3), 484-550 (1994)

75. Sjöstrand, J., Zworski, M.: Elementary linear algebra for advanced spectral problems. Ann. Inst. Fourier (Grenoble) 57(7), 2095-2141 (2007)

76. Smale, S.: An Infinite Dimensional Version of Sard's Theorem. Amer. J. Math. 87(4), 861-866 (1965)

77. Subramaniam, T.N.: Slices for the Actions of Smooth Tame Lie Groups. PhD Thesis, Brandeis University (1984)

78. Subramaniam, T.N.: Slices for actions of infinite-dimensional groups. In: Differential Analysis in Infinite Dimensional Spaces, vol. 54, American Mathematical Society, pp. 65-77 (1986)

79. Taubes, C.H.: Self-dual Yang-Mills connections on non-self-dual 4-manifolds. J. Differential Geom. 17(1), 139-170 (1982)

80. Teichmann, J.: A Frobenius Theorem on Convenient Manifolds. Monatshefte für Mathematik 134(2), 159-167 (2001)

81. Yang, D.: A Choice-independent Theory of Kuranishi Structures and the Polyfold-kuranishi Correspondence. PhD Thesis. New York University (2014)

Publisher's Note Springer Nature remains neutral with regard to jurisdictional claims in published maps and institutional affiliations. 